\title{
Inventario florístico del Parque Nacional Cañón del Sumidero, Chiapas, MéXico
}

\author{
Josefa Anahí Espinosa-Jiménez', Miguel Ángel Pérez-Farrera y Rubén Martínez-Camilo \\ Herbario Eizi Matuda, Facultad de Ciencias Biológicas, Universidad de Ciencias y Artes de Chiapas \\ 1Autor para la correspondencia: anahi_espinosa_jimenez@yahoo.com.mx
}

\begin{abstract}
Resumen: Se realizó el inventario florístico del Parque Nacional Cañón del Sumidero, Chiapas, México. Treinta y tres salidas de campo se hicieron de 2007 a 2008 y se consultaron y revisaron bases de datos de herbarios. Se registraron 1,298 especies, 632 géneros, 135 familias y 58 infraespecies. Las familias más representativas corresponden a Fabaceae (126 especies y 52 géneros) y Asteraceae (107 especies y 65 géneros). Los géneros más diversos fueron Ipomoea (18), Tillandsia (17) y Peperomia (16). Además, 625 especies se clasificaron como hierbas y 1,179 especies como autótrofas. El bosque tropical caducifolio fue el más diverso con 30.91\% de las especies reportadas. Dentro de alguna categoría de riesgo dentro de la NOM-ECOL-059-2010 se registraron 24 especies y $2.6 \%$ de la flora del Cañón del Sumidero es endémica de Chiapas. Dos especies, Valeriana palmeri A. Gray y Dioon sp., posiblemente están extintas en el área de estudio.

Palabras clave: área natural protegida, base de datos, bosque tropical caducifolio, endemismo, riqueza.
\end{abstract}

\begin{abstract}
A floristic inventory was conducted in the Sumidero Canyon National Park, Chiapas, Mexico. Thirty-three field trips were conducted from 2007 to 2008 and the revision of herbaria data bases. About 1,298 species and 58 infraspecies were recorded among 632 genera and 135 families. The most representative families were Fabaceae (126 species with 52 genera) and Asteraceae (with 107 species and 65 genera). The most diverse genera were Ipomoea (18), Tillandsia (17), and Peperomia (16). In addition, 625 species were classified as herbs and 1,179 species were terrestrial autotrophic. Tropical deciduous forest was the most diverse with $30.91 \%$ of the species reported. About 24 species were recorded under some level of threat-risk within the official threatened species list NOM-ECOL-059-2010 and 2.6\% of the flora reported in the Sumidero Canyon is endemic to Chiapas. Two species, Valeriana palmeri A. Gray and Dioon sp., are thought possibly extinct in the study area.
\end{abstract}

Keywords: database, endemism, protected natural area, richness, tropical deciduous forest.

os parques nacionales constituyen la categoría de área Lnatural protegida más conocida y de mayor arraigo en el mundo; la motivación principal para declarar un área como parque nacional consideraba en un principio valores de tipo histórico, turístico, estético o incluso político, pero visto desde el ángulo de la sociedad urbana; el aspecto biológico, en esos inicios prácticamente no influyó (Gómez-Pompa y Dirzo, 1995). En fechas recientes se incorporan nuevos elementos que consideran el valor científico, educativo, de recreo, histórico y la diversidad de la flora y fauna; así como su aptitud para el desarrollo del turismo (Gómez-Pompa y Dirzo, 1995). El 24 de mayo de 1972 el gobierno del Estado de Chiapas decretó como parque a las tierras que constitu- yen el Cañón del Sumidero (Olay-Barrientos, 1987). Posteriormente, considerando que cuenta con una belleza natural imponente en México y que reúne condiciones singulares por la variedad de fauna y flora, clima, topografía y el alto valor geológico, el ocho de diciembre de 1980 fue decretado como Parque Nacional "Cañón del Sumidero" (GómezPompa y Dirzo, 1995). Adicionalmente, el área en donde se encuentra el Parque Nacional Cañón del Sumidero es muy importante para la conservación de biodiversidad, por lo que en el año 2004 la Convención sobre los Humedales de Importancia Internacional, llamada Convención de RAMSAR, designó al Cañón del Sumidero como sitio RAMSAR por sustentar especies vulnerables y en peligro de extinción 
(Arriaga-Cabrera et al., 2009). La Comisión Nacional para el Conocimiento y Uso de la Biodiversidad (CONABIO) designó a la zona La Chacona-Cañón del Sumidero como "Región Prioritaria Terrestre". El Cañón del Sumidero, también forma parte del corredor Laguna Bélgica-Sierra LimónCañon Sumidero, la cual es considerada como un área de importancia para la conservación de las aves (Arizmendi y Marquez, 2000; Arriaga et al., 2000). Adicionalmente, su belleza escénica es de gran valor estético, por lo cual tiene una gran demanda turística, ingresando miles de turistas al año. Sin embargo, la gran afluencia turística, su cercanía con la capital de Chiapas (Tuxtla Gutiérrez) y municipios conurbados (Chiapa de Corzo, Chicoasén y San Fernando) y los problemas ambientales como la expansión de la frontera agrícola y asentamientos humanos, los incendios forestales, y la contaminación de cuerpos y corrientes de agua, son considerados como las amenazas más importantes para los ecosistemas del Parque Nacional Cañón del Sumidero (March-Mifsut y Flamenco-Sandoval, 1996; CONANP, 2007).

Los estudios para conocer la diversidad biológica del Parque Nacional Cañón del Sumidero han sido pocos y se han enfocado principalmente en conocer la diversidad faunística. Estos estudios han demostrado la importancia del Parque Nacional Cañón del Sumidero como área natural protegida al resguardar un número importante de vertebrados bajo alguna categoría de riesgo (Gálvez, 1990; Altamirano-González-Ortega et al., 2008; García-Marmolejo et al., 2008). Florísticamente el Parque Nacional Cañón del Sumidero ha sido colectado, pero no de manera suficiente a pesar de ser un área relativamente accesible por su cercanía con Tuxtla Gutiérrez, debido a áreas de acceso poco conocidas e inexploradas florísticamente. El único trabajo florístico realizado hasta el momento que incluyó sitios de colecta dentro del Cañón del Sumidero es el de Reyes-García y Sousa (1997). Sin embargo, en este estudio sólo se realizaron colectas en selva baja caducifolia. También se reportan numerosas colectas botánicas hechas por importantes colectores de la flora Chiapaneca como Faustino Miranda, Dennis Breedlove y Esteban Martínez (Reyes-García y Sousa 1997; González-Espinosa et al., 2005). Ante el desconocimiento de la riqueza florística de esta área natural protegida, se requiere la integración de todas las colectas botánicas que se han realizado hasta el momento; además de explorar en aquellas áreas de las que no se tienen registros de herbario. Es por ello que nos propusimos en este trabajo realizar el inventario florístico del Parque Nacional Cañón del Sumidero, con lo cual se contribuye al conocimiento de la riqueza florística que se mantiene y resguarda en esta área natural. Los datos generados aportarán información de distribución y fenología de taxones que están bajo alguna categoría de protección por las leyes nacionales o internacionales y podrán ayudar a los responsables del Parque a poner mayores esfuerzos en la conservación y el monitoreo.

\section{Materiales y métodos}

Zona de estudio. El Parque Nacional Cañón del Sumidero está ubicado entre los $16^{\circ} 44^{\prime}$ y $16^{\circ} 56^{\prime}$ de latitud Norte y los $93^{\circ} 00^{\prime}$ y los $93^{\circ} 11^{\prime}$ de longitud Oeste, con altitudes que van de 360 a 1,720 m s.n.m. Se localiza entre los municipios de Tuxtla Gutiérrez, Chiapa de Corzo, San Fernando, Osumacinta, Chicoasén, Soyaló e Ixtapa y tiene una extensión de 21,789 ha (Pronatura-Chiapas, 2002). El Parque Nacional Cañón del Sumidero está situado en terrenos de dos regiones fisiográficas, la Depresión Central y la Altiplanicie Central y limita en una pequeña porción con las Montañas del Norte (Müllerried, 1957). Los tres tipos de clima presentes en el parque son: Awo(w)(i)g que es un clima cálido, el más seco de todos los cálidos subhúmedos con lluvias en verano. El porcentaje de lluvia invernal es menor al 5\% del total anual. Presenta una temperatura media anual sobre los $25^{\circ} \mathrm{C}$, la temperatura del mes más cálido del año antes de junio; C(wi)(i)g, semicálido, es el más cálido de los templados, con temperatura media anual mayor a los $18{ }^{\circ} \mathrm{C}$, lluvias en verano, precipitación invernal entre 5 y $92 \%$ respecto al total anual y Awi(w)ig, temperatura media anual mayor a los $22{ }^{\circ} \mathrm{C}$ y la del mes más frío mayor a $18{ }^{\circ} \mathrm{C}$, precipitación invernal menor al 5\% del total anual (García, 1964). Se reportan cuatro tipos principales de suelos: (1) Litosoles, (2) Regosoles diferenciados en los subtipos Regosoles éutricos y Regosoles calcáreos, (3) Luvisoles crómicos y (4) Rendzinas (INEGI, 1985). Gran parte de los suelos en la zona se caracterizan por su grado de desarrollo relativamente bajo, una someridad más o menos pronunciada y por su composición química básica, debido a los altos contenidos en carbonato de calcio y de los nutrientes básicos de calcio, magnesio y potasio. El Parque Nacional Cañón del Sumidero se conforma por tres sistemas hidrológicos: el sistema hidrológico cárstico de las mesetas calcáreas que colindan con el Cañón del Sumidero; el del río Grijalva y el de la presa de Chicoasén (CONANP, 2007). El principal sistema hidrológico del Parque es el río Grijalva, el cual, después de dejar la Depresión Central Chiapaneca, pasa a través del Cañón del Sumidero y cruza las fallas "Muñiz" y "Chicoasén", ésta última en donde se encuentra la cortina de la presa hidroeléctrica Manuel Moreno Torres. El ancho del cañón varía de uno a dos kilómetros (Pronatura-Chiapas, 2002).

Método. Se realizaron 33 salidas de campo entre el 21 de junio de 2007 al 27 de junio de 2008 dentro del polígono del Parque Nacional Cañón del Sumidero. Los puntos de colecta se escogieron por el tipo de vegetación y topografía, cubriendo todos los tipos de vegetación y las áreas mejor conservadas. Cada ejemplar se colectó y proceso de acuerdo al procedimiento de Lot y Chiang (1986). Se colectaron cuatro juegos de cada ejemplar, el primero se depositó en el HEM y los posteriores en los herbarios MEXU, MO, CAS. La identificación a nivel de especie se realizó con ayuda 


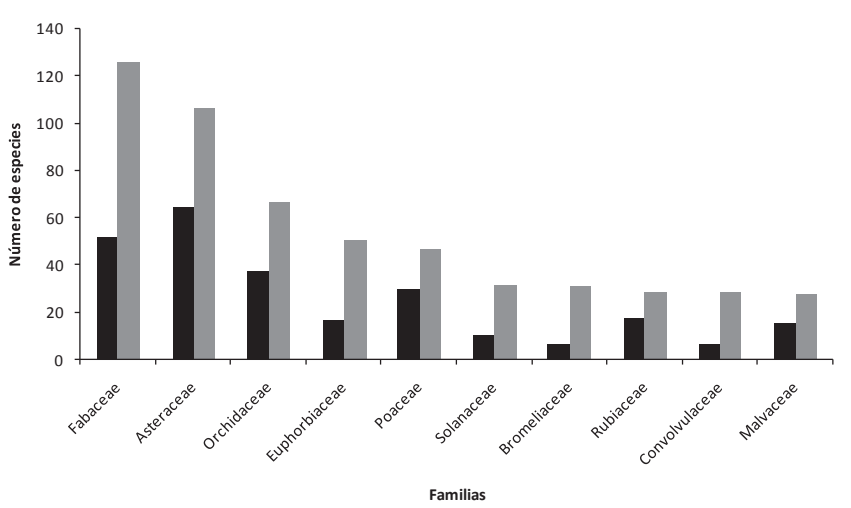

Figura 1. Relación de familias con mayor número de géneros (barras negras) y especies (barras grises) del Parque Nacional Cañón del Sumidero, Chiapas.

de bibliografía especializada y de especialistas en diversas familias. Adicionalmente, se revisaron las bases de datos en internet y ejemplares de herbario del CAS, CHIP, DS, ENCB, HEM, IEB, IE-XAL, IPN, LL-TEX, MEXU, MO y UAMIZ. Toda la información de las colectas realizadas y consultadas se integraron en una base de datos en Microsoft Access 2007. Las formas de crecimiento (árbol, arborescente, arbusto, hierba, columnar, liana o bejuco) y formas de nutrición (acuática, autótrofa, epífita, hemiepífita, parásita, rupícola, saprófita) se tomaron de Hickey y King (2000) con algunas modificaciones. Se determinó el estado de conservación usando el criterio de la lista roja de la Unión Internacional para la Conservación de la Naturaleza (IUCN, 2010) y la Norma Oficial Mexicana (SEMARNAT, 2010).

\section{Resultados}

Se conformó una base de datos con 2,902 registros de herbario de colectas realizadas dentro del Parque Nacional Cañón del Sumidero. Se reportan 135 familias, 632 géneros, 1,298

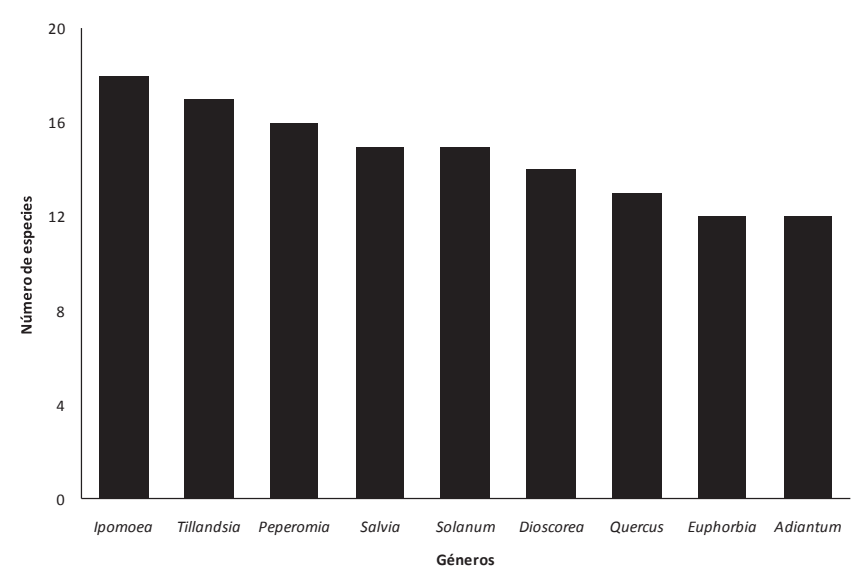

Figura 2. Relación de géneros más abundantes en el Parque Nacional Cañón del Sumidero, Chiapas.
Cuadro 1. Datos generales sobre la flora vascular del Parque Nacional Cañón del Sumidero, Chiapas.

\begin{tabular}{lcccc}
\hline División & Familias & Géneros & Especies & Infraespecies \\
\hline Gymnospermae & 4 & 5 & 5 & \\
Liliopsida & 20 & 115 & 239 & 8 \\
Magnoliopsida & 100 & 481 & 971 & 6 \\
Pteridophyta & 11 & 31 & 83 & 4 \\
TOTAL & 135 & 632 & 1,298 & 8 \\
\hline
\end{tabular}

especies y 58 infraespecies (Cuadro 1). El grupo mejor representado fue Magnoliopsida con 971 (74.8\%) especies. Las familias más diversas fueron Fabaceae con 52 géneros y 126 especies y Asteraceae con 65 géneros y 107 especies (Figura 1). Los géneros más diversos fueron Ipomoea, Tillandsia, y Peperomia (Figura 2). Por forma de crecimiento, las hierbas fueron las más abundantes con 625 especies y las columnares fueron las menos abundantes con sólo cuatro especies. El tipo de nutrición más común es el autótrofo con 1,179 especies, dentro de las menos frecuentes están las parásitas, hemiepífitas, acuáticas y las saprófitas (Apéndice 1). De los siete tipos de vegetación presentes, los más diversos en especies de plantas fueron el bosque tropical caducifolio, el bosque tropical perennifolio y el bosque tropical subcaducifolio con $30.91 \%, 29.34 \%$ y $28.35 \%$ de las especies colectadas respectivamente (Figura 3). De las especies de flora endémicas, se encontraron 34 endémicas de Chiapas, 36 de distribución restringida (una restringida a Chiapas, Belice y Oaxaca; una de Chiapas y Guerrero; dos de Chiapas, Guatemala y Veracruz; dos de Chiapas, Oaxaca y Veracruz; seis de Chiapas y Veracruz; seis de Chiapas, Guatemala y Oaxaca; nueve de Chiapas y Guatemala y nueve de Chiapas y Oaxaca) y 42 de México a Centroamérica (Apéndice 1). Se

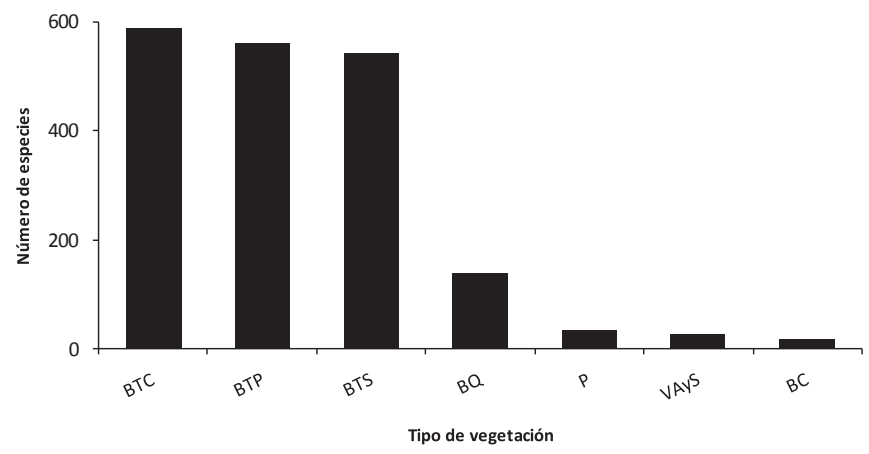

Figura 3. Porcentajes de la riqueza de especies de los tipos de vegetación presentes en el Parque Nacional Cañón del Sumidero, Chiapas. BTC: bosque tropical caducifolio, BTP: bosque tropical perennifolio, BTS: bosque tropical subcaducifolio, BQ: bosque de Quercus, P: pastizal, VAyS: vegetación acuática y subacuática, BC: bosque de coniferas. 
Cuadro 2. Especies del Parque Nacional Cañón del Sumidero que se encuentran bajo alguna categoría de riesgo en la NOM-ECOL-059-2010.

\begin{tabular}{|c|c|c|c|}
\hline Especie & Familia & Estatus & Distribución \\
\hline Agave grijalvensis B. Ullrich & Agavaceae & $\operatorname{Pr}$ & Endémica \\
\hline Astronium graveolens Jacq. & Anacardiaceae & A & No endémica \\
\hline Monstera tuberculata Lundell & Araceae & A & No endémica \\
\hline Brahea nitida André & Arecaceae & $\operatorname{Pr}$ & No endémica \\
\hline Chamaedorea arenbergiana $\mathrm{H}$. Wendl. & Arecaceae & A & No endémica \\
\hline Chamaedorea ernesti-augusti $\mathrm{H}$. Wendl. & Arecaceae & A & No endémica \\
\hline Chamaedorea glaucifolia H. Wendl. & Arecaceae & $\mathrm{P}$ & Endémica \\
\hline Chamaedorea stolonifera H. Wendl. ex Hook. f. & Arecaceae & A & Endémica \\
\hline Tabebuia chrysantha G. Nicholson & Bignoniaceae & A & No endémico \\
\hline Catopsis berteroniana (Schult. \& Schult. f.) Mez & Bromeliaceae & $\operatorname{Pr}$ & No endémica \\
\hline Tillandsia concolor L.B. Sm. & Bromeliaceae & A & No endémica \\
\hline Tillandsia seleriana Mez & Bromeliaceae & A & No endémica \\
\hline Tillandsia tricolor Schltdl. \& Cham. & Bromeliaceae & A & No endémica \\
\hline Bursera arborea (Rose) L. Riley & Burseraceae & A & Endémica \\
\hline Litsea glaucescens Kunth & Lauraceae & $\mathrm{P}$ & No endémica \\
\hline Beaucarnea goldmanii Rose & Liliaceae & A & Endémica \\
\hline Cedrela odorata L. & Meliaceae & $\operatorname{Pr}$ & No endémica \\
\hline Epidendrum skutchii Ames, F.T. Hubb. \& C. Schweinf. & Orchidaceae & $\operatorname{Pr}$ & No endémica \\
\hline Guarianthe skinneri (Bateman) Dressler \& W.E. Higgins & Orchidaceae & A & No endémica \\
\hline $\begin{array}{l}\text { Pseudogoodyera pseudogoodyeroides (L.O. Williams) } \\
\text { R. González \& Szlach. }\end{array}$ & Orchidaceae & $\operatorname{Pr}$ & No endémica \\
\hline Sarcoglottis cerina (Lindl.) P.N. Don & Orchidaceae & $\operatorname{Pr}$ & No endémica \\
\hline Serpocaulon triseriale (Sw.) A.R. Sm. & Polypodiaceae & A & No endémica \\
\hline Sideroxylon capiri (A.DC.) Pittier & Sapotaceae & A & No endémica \\
\hline Ceratozamia robusta Miq. & Zamiaceae & A & Endémica \\
\hline
\end{tabular}

encontraron 24 especies de flora incluidas en alguna categoría de riesgo según la Norma Oficial Mexicana (Cuadro 2) y 17 especies se encuentran en la Lista Roja de especies en peligro de la Unión Internacional para la Conservación de la Naturaleza (Cuadro 3), de acuerdo a esta lista, Lonchocarpus minimiflorus Donn. Sm., Parathesis vulgata Lundell y Wimmeria montana Lundell se encuentran en peligro de extinción.

\section{Discusión}

El Parque Nacional Cañón del Sumidero alberga una riqueza de flora que equivale al $15.7 \%$ de la flora estimada para Chiapas (Breedlove, 1986). Considerando la extensión territorial, su ubicación geográfica y el tipo de área natural, es posible señalar que la diversidad florística encontrada para el Parque Nacional Cañón del Sumidero es relevante e importante (Cuadro 4). Por el momento supera la riqueza floristica reportada por Reyes-García y Sousa (1997) para la selva baja caducifolia de la Depresión Central de Chiapas, tiene casi dos terceras partes de la diversidad florística de la Sepultura (Reyes-García, 2008) y una tercera parte de la reportada para El Triunfo (Pérez-Farrera, 2004), aunque ambas son reservas de la biosfera y tienen una extensión te- rritorial considerablemente mayor. Las familias Asteraceae y Fabaceae son las más diversas en el Parque Nacional Cañón del Sumidero concordando con lo encontrado por Castelo et al. (2005), Sousa-S. y Delgado-S. (1998) y Villaseñor (2003) como las familias más abundantes en México. Los géneros con mayor número de especies son Ipomoea, Peperomia y Tillandsia, tales géneros se encuentran entre los más abundantes de México (Villaseñor, 2004) y son característicos de los bosques tropicales caducifolios.

Rzedowski $(1978,1998)$ menciona que el bosque tropical caducifolio es un ecosistema que tiene un componente endémico muy importante. Sin embargo, en este trabajo sólo se encontraron cuatro especies endémicas para este tipo de vegetación: Agave grijalvensis B. Ullrich, Ateleia chicoasensis J. Linares, Hybosema robustum M. Sousa \& Lavin y Lonchocarpus martinezii M. Sousa. En contraste, el número de especies endémicas de Chiapas y que se encuentran en el Parque Nacional Cañón del Sumidero es elevado con 34 especies; entre ellas destacan especies con una distribución limitada a la cuenca del río Grijalva como Anthurium clarinervium Matuda, A. faustomirandae Pérez-Farrera \& Croat, Dioscorea sumiderensis B.G. Schub. \& O. Téllez y Pitcairnia breedlovei L.B. Sm. Algunas especies de la flora son saqueadas en muchas áreas dentro y cercanas al Parque, 
Cuadro 3. Especies del Parque Nacional Cañón del Sumidero que se encuentran en la lista roja de especies en Peligro.

\begin{tabular}{|c|c|c|}
\hline Especie & Familia & Estatus IUCN \\
\hline $\begin{array}{l}\text { Wimmeria montana } \\
\text { Lonchocarpus minimiflorus } \\
\text { Pararathesis vulgata }\end{array}$ & $\begin{array}{l}\text { Celastraceae } \\
\text { Fabaceae } \\
\text { Myrsinaceae }\end{array}$ & $\begin{array}{l}\text { Endangered } \\
\text { (en peligro) }\end{array}$ \\
\hline Taxodium mucronatum & Taxodiaceae & $\begin{array}{l}\text { Least Concern } \\
\text { (preocupación menor) }\end{array}$ \\
\hline Arbutus xalapensis & Ericaceae & $\begin{array}{l}\text { Lower Risk/ } \\
\text { conservation } \\
\text { dependent } \\
\text { (dependiente de } \\
\text { conservación) }\end{array}$ \\
\hline $\begin{array}{l}\text { Crossopetalum parvifolium } \\
\text { Quercus sebifera } \\
\text { Ficus trigonata } \\
\text { Pinus oocarpa }\end{array}$ & $\begin{array}{l}\text { Celastraceae } \\
\text { Fagaceae } \\
\text { Moraceae } \\
\text { Pinaceae }\end{array}$ & $\begin{array}{l}\text { Lower Risk/least } \\
\text { concern (preocupación } \\
\text { menor) }\end{array}$ \\
\hline $\begin{array}{l}\text { Brahea nítida } \\
\text { Juniperus gamboana } \\
\text { Quercus benthamii } \\
\text { Quercus purulhana } \\
\text { Robinsonella mirandae } \\
\text { Cedrela odorata } \\
\text { Swietenia humilis } \\
\text { Ceratozamia robusta }\end{array}$ & $\begin{array}{l}\text { Arecaceae } \\
\text { Cupressaceae } \\
\text { Fagaceae } \\
\text { Fagaceae } \\
\text { Malvaceae } \\
\text { Meliaceae } \\
\text { Meliaceae } \\
\text { Zamiaceae }\end{array}$ & Vulnerable \\
\hline
\end{tabular}

Cuadro 4. Comparación de la riqueza florística del Parque Nacional Cañón del Sumidero con otras áreas naturales protegidas o regiones.

\begin{tabular}{|c|c|c|c|}
\hline Área Natural Protegida & Superficie & $\begin{array}{l}\text { Número } \\
\text { especies }\end{array}$ & Fuente \\
\hline $\begin{array}{l}\text { Parque Nacional Cañón } \\
\text { del Sumidero }\end{array}$ & 21,789 ha & 1,298 & Este trabajo. \\
\hline $\begin{array}{l}\text { Parque Educativo } \\
\text { Laguna Bélgica }\end{array}$ & 47.5 ha & 254 & $\begin{array}{l}\text { Escobar-Ocampo } \\
\text { y Ochoa-Gaona. } \\
2007 .\end{array}$ \\
\hline $\begin{array}{l}\text { Reserva de la Biosfera } \\
\text { El Ocote }\end{array}$ & 48,000 ha & 705 & $\begin{array}{l}\text { Ochoa-Gaona, } \\
1996 .\end{array}$ \\
\hline $\begin{array}{l}\text { Reserva de la Biosfera } \\
\text { El Triunfo }\end{array}$ & 119,117 ha & 3,000 & $\begin{array}{l}\text { Pérez-Farrera, } \\
2004\end{array}$ \\
\hline $\begin{array}{l}\text { Reserva de la Biosfera } \\
\text { La Sepultura }\end{array}$ & 167,309 ha & 1,798 & $\begin{array}{l}\text { Reyes-García, } \\
2008 .\end{array}$ \\
\hline $\begin{array}{l}\text { Selva Baja Caducifolia } \\
\text { de la Depresión Central } \\
\text { de Chiapas }\end{array}$ & 892,980 ha & 988 & $\begin{array}{l}\text { Reyes-García y } \\
\text { Sousa } 1997\end{array}$ \\
\hline Chiapas & $7,441,500$ ha & 8,248 & Breedlove, 1986. \\
\hline
\end{tabular}

porque son muy llamativas por su belleza ornamental y se encuentran en alguna categoría de riesgo, principalmente la orquídea Guarianthe skinneri (Bateman) Dressler \& W.E.
Higgins, las palmas Chamaedorea arenbergiana $\mathrm{H}$. Wendl., C. ernesti-augusti H. Wendl. y C. glaucifolia H. Wendl., la cícada Ceratozamia robusta Miq, Beaucarnea goldmanii Rose llamada comúnmente Pata de elefante, Pilosocereus leucocephalus (Poselgar) Byles G.D. Rowley (cabeza de viejo), las aráceas Anthurium clarinervium Matuda y A. seleri Engl. y Agave grijalvensis B. Ullrich, cuyas hojas se utilizan para preparar barbacoas, por lo cual es saqueada ilegalmente. Otras plantas son importantes por su uso maderable como Astronium graveolens Jacq. y Tabebuia chrysantha (Jacq.) G. Nicholson, ambas catalogadas en la norma ecológica mexicana como amenazadas. Se tienen los registros de dos especies posiblemente extintas en el área; una de ellas es Valeriana palmeri A. Gray. (Valerianaceae), la cual también es reportada como extinta para Veracruz (Vovides et al., 1997). Esta especie fue colectada por Denis E. Breddlove en 1965 y desde ese entonces no se ha vuelto a colectar. Así también, se tiene registro de una especie de Dioon sp. colectada por Marcos E. Becerra en 1920, para la cual se han realizado intensas búsquedas dentro del Parque pero no se ha encontrado, por lo que se presume se encuentra extinta. Adicionalmente, se sabe por pláticas informales que los habitantes de las comunidades asentadas dentro del Parque Nacional Cañón del Sumidero, hace más de 50 años extrajeron esta planta intensivamente para venderla, lamentablemente esta especie nunca fue descrita.

La riqueza florística encontrada en este trabajo fue mayor a lo reportado para otras zonas adyacentes y áreas naturales protegidas, encontrado elementos florísticos que son endémicos, amenazados o sujetos a algún aprovechamiento. Se sugiere la implementación de planes de conservación y monitoreo de especies de la flora en zonas donde más presión humana se ejerce dentro del polígono del Parque, así como de estrategias de conservación y aprovechamiento de aquellas que por su belleza ornamental así lo requieren, como las especies de palmas (Chamaedorea spp.), aráceas (Araceae) y orquídeas (Orchidaceae) y la denominada pata de elefante (Beaucarnea goldmanii).

\section{Agradecimientos}

La primera autora agradece a la dirección del Parque Nacional Cañón del Sumidero, a la Fundación Dr. Christopher y Sharon Davidson por el financiamiento otorgado (proyecto IFT01/05) y al programa de becas de la Secretaria de Educación Pública por la beca otorgada bajo el concepto de Beca de Titulación (Convenio DIE: 0814970). El segundo autor agradece al fondo PROMEP (PROMEP/103.5/07/2363) por haber otorgado una beca de tesis. Los autores agradecen a Andrew P. Vovides por la revisión del abstract. Ayudaron en la determinación de ejemplares Carlos Rommel Beutelspacher (Orquidaceae y Bromeliaceae), Martha Martínez Gordillo (Euphorbiaceae y Lamiaceae), Charlotte Taylor (Rubiaceae), Ramiro Cruz Durán (Fabaceae), Adolfo Es- 
pejo Serna (Bromeliaceae), Mario Sousa y Lourdes Rico (Fabaceae) W.D. Stevens (Apocynaceae y Asclepiadaceae), John F. Pruski (Asteraceae) y Oscar Farrera Sarmiento (varias familias).

\section{Literatura citada}

Altamirano-González-Ortega M.A., Riechers-Pérez A., Luna-Reyes R., Guzmán-Hernández J. y Vidal-López R. 2008. Parque Nacional Cañón del Sumidero: refugio de vertebrados terrestres. Biodiversitas 80:12-15.

Arizmendi M. C. y Márquez V.L. 2000. Áreas de Importancia para la Conservación de las Aves. Consejo Internacional para la Preservación de las Aves (CIPAMEX), México.<http://conabioweb.conabio.gob.mx/aicas/doctos/aicaslista.html>. (Consultado 6 de agosto de 2010).

Arriaga-Cabrera L., Aguilar V. y Espinoza J.M. 2009. Regiones prioritarias y planeación para la conservación de la biodiversidad. En: Dirzo R., González R. y March I.J. Coords. Capital Natural de México, Volumen II: Estado de Conservación y Tendencias de Cambio, pp. 443-458, Comisión Nacional para el Conocimiento y Uso de la Biodiversidad. México, D.F.

Arriaga L., Espinoza J.M., Aguilar C., Martínez E., Gómez L. y Loa E. Coords. 2000. Regiones Terrestres Prioritarias de México. Comisión Nacional para el Conocimiento y uso de la Biodiversidad. México, D.F. <www.conabio.gob.mx/conocimiento/ regionalizacion/doctos/rtp_141.pdf > (consultado 9 de agosto de 2010).

Breedlove D.E. 1986. Listados Florísticos de México VI Flora de Chiapas. Instituto de Biología, Universidad Nacional Autónoma de México. México, D.F.

Castelo E., Ricalde O. y Panero J. 2005. Catálogo de Autoridades de Asteráceas Mexicanas y Actualización de Tribus Heliantheae y Eupatorieae. University of Texas. Base de datos SNIB-CONABIO México, D.F.

CONANP [Comisión Nacional de Áreas Naturales Protegidas]. 2007. Estudio Previo Justificativo para Modificar el Decreto del Área Natural Protegida Parque Nacional Cañón del Sumidero, Chiapas, México. Comisión Nacional de Áreas Naturales Protegidas. México, D.F.

Escobar-Ocampo M.C. y Ochoa-Gaona S. 2007. Estructura y composición florística de la vegetación del Parque Educativo Laguna Bélgica, Chiapas. México. Revista Mexicana de Biodiversidad 78:391-419.

Gálvez J. 1990. Mastofauna del Parque Nacional Cañón del Sumidero Chiapas, México. Tesis de Licenciatura, Escuela de Biología, Instituto de Ciencias y Artes de Chiapas, Tuxtla Gutiérrez, 89 pp.

García E. 1964. Modificaciones al Sistema de Clasificación Climática de Köeppen (para Adaptarlo a las Condiciones de la República Mexicana). Offset Larios S.A. México, D.F.

García-Marmolejo G., Escalante T. y Morrone J.J. 2008. Establecimiento de prioridades para la conservación de mamíferos terrestres neotropicales de México. Mastozoología Neotropical 15:41-65.

Gómez-Pompa A. y Dirzo R. 1995. Reservas de la Biosfera y otras Áreas Naturales Protegidas de México. Secretaría de Medio Ambiente, Recursos Naturales y Pesca-Instituto Nacional de Ecología-Comisión Nacional para el Conocimiento y Uso de la Biodiversidad. México, D.F.
González-Espinosa M., Ramírez-Marcial N., Méndez-Dewar G., Galindo-Jaimes L. y Golicher D. 2005. Riqueza de especies de árboles en Chiapas: variación espacial y dimensiones ambientales asociadas al nivel regional. En: González-Espinosa M., Ramírez-Marcial N. y Ruíz-Montoya L. Coords. Diversidad Biológica de Chiapas, pp. 81-125, Plaza y Valdés, México, D.F.

Hickey M. y King C. 2000. The Cambridge Illustrated Glossary of Botanical Terms. Cambridge University Press, Cambridge.

INEGI [Instituto Nacional de Estadística, Geografía e Informática]. 1985. Carta Edafológica 1:250 000 Tuxtla Gutiérrez E1511, Proyección transversa de Mercator. Instituto Nacional de Estadística, Geografía e Informática, México, D.F.

IUCN [International Union for Conservation of Nature]. 2010. IUCN Red List of Threatened Species. Version 2010.4. <www. iucnredlist.org>. (consultado del 1-30 de agosto de 2010).

Lot A. y Chiang F. Comps. 1986. Manual de Herbario. Administración y Manejo de Colecciones, Técnicas de Recolección y Preparación de Ejemplares Botánicos. Consejo Nacional de la Flora de México. A.C. México, D.F.

March-Mifsut I.J. y Flamenco-Sandoval A. 1996. Evaluación rápida de la deforestación en las áreas naturales protegidas de Chiapas (1970-1993). El Colegio de la Frontera Sur, San Cristobal de las Casas.

Müllerried F.K.G. 1957. Geología de Chiapas. Gobierno del Estado de Chiapas, Tuxtla Gutiérrez.

Ochoa-Gaona S. 1996. La vegetación de La Reserva El Ocote a lo largo del cañón del río La Venta. En: Vásquez-Sánchez M.A. y March-Mifsut I. Eds. Conservación y Desarrollo Sustentable en la Selva El Ocote, Chiapas, pp. 45-86, El Colegio de la Frontera Sur-Comisión Nacional para el Conocimiento y Uso de la Biodiversidad-Centro de Estudios para la Conservación de los Recursos Naturales, A.C., San Cristóbal de Las Casas.

Olay-Barrientos M.A. 1987. El Sumidero, Chiapas: un sitio del Clásico Tardío. Gobierno del Estado de Chiapas Consejo Estatal de Fomento a la Investigación y Difusión de la Cultura DIFCHIAPAS, Instituto Chiapaneco de Cultura, Tuxtla Gutiérrez.

Pérez-Farrera M.A. 2004. Flora y vegetación de la Reserva de la Biosfera El Triunfo: Diversidad, riqueza y endemismo. En: Pérez-Farrera M.A., Martínez-Meléndez N., Hernández-Yáñez A. y Arreola-Muñoz A.V. Eds. La Reserva de la Biosfera El Triunfo, Tras Una Década de Conservación, pp. 77-100, Universidad de Ciencias y Artes de Chiapas, México, D.F.

Pronatura-Chiapas. 2002. Programa de manejo del Parque Nacional Cañón del Sumidero, Chiapas. San Cristóbal de las Casas. (Documento inédito)

Reyes-García A. y Sousa-S M. 1997. Listados Florísticos de México XVII. Depresión Central de Chiapas. La Selva Baja Caducifolia. Instituto de Biología, Universidad Nacional Autónoma de México, México, D.F.

Reyes-García A.J. 2008. Inventario florístico de la reserva de la biosfera La Sepultura, Sierra Madre de Chiapas. Tesis de Maestría, Facultad de Ciencias, Universidad Nacional Autónoma de México, México, D.F. 195 pp.

Rzedowski J. 1978. Vegetación de México. Limusa, México, D.F.

Rzedowski J. 1998. Diversidad y orígenes de la flora fanerogámica de México. En: Ramamoorthy T.P., Bye R., Lot A. y Fa J. Comps. Diversidad Biológica de México: Orígenes y Distribución, pp. 129-145, Instituto de Biología, Universidad Autónoma de México. México, D.F.

SEMARNAT [Secretaria del Medio Ambiente y Recursos Natu- 
rales]. 2010. Norma Oficial Mexicana NOM-059-SEMARNAT-2010, Protección ambiental - Especies nativas de México de Flora y Fauna Silvestres - Categorías de Riesgo y especificaciones para su inclusión, exclusión o cambio - Lista de Especies en Riesgo. Diario Oficial de la Federación 2a Sección, 30 de diciembre del 2010. <www.semarnat.gob. mx/leyesynormas/ SEMARNAT\%20DOF/Norma\%20Oficial\%20Mexicana $\% 20$ NOM-059-SEMARNAT-2010.pdf>

Sousa-S M. y Delgado-S A. 1998. Leguminosas mexicanas: fitogeografía, endemismo y orígenes. En: Ramamoorthy T.P., Bye R., Lot A. y Fa J. Comps. Diversidad Biológica de México Orí- genes y Distribución, pp. 449-500, Instituto de Biología, Universidad Nacional Autónoma de México, México, D.F.

Villaseñor J.L. 2003. Diversidad y distribución de las Magnoliophyta de México. Interciencia 28:160-167.

Villaseñor J.L. 2004. Los géneros de plantas vasculares de la flora de México. Boletín de la Sociedad Botánica de México 75:105135.

Vovides A.P., Luna V. y Medina G. 1997. Relación de algunas plantas y hongos mexicanos raros, amenazados o en peligro de extinción y sugerencias para su conservación. Acta Botanica Mexicana 39:1-42.

Recibido: 11 de enero de 2011

Aceptado: 18 de mayo de 2011 
Apéndice 1. Lista florística de la vegetación del Parque Nacional Cañón del Sumidero, Chiapas, México.

Forma de crecimiento (FC): Á = Árbol, Ar = Arborescente, Ab = Arbusto, $\mathbf{C l}=$ Columnar, $\mathbf{H i}=$ Hierba, Li $=$ Liana o bejuco. Forma de nutrición $(\mathbf{F N})$ : Ac = Acuática, Au = Autótrofa, $\mathbf{E p}=$ Epífita, $\mathbf{H e}=$ Hemiepífita, $\mathbf{R u}=$ Rupícola, $\mathbf{P a}=$ Parásita, $\mathbf{S a}=$ Saprófita. Fenología $(\mathbf{F E}): \mathbf{E}=$ Estéril, $\mathbf{F e}=$ Fértil $($ Helechos), $\mathbf{F I}=$ Flor, $\mathbf{F r}=$ Fruto. Mes de colecta $(\mathbf{M C}): 1=$ Enero hasta $12=$ Diciembre. Estatus de protección en la NOM-059-SEMARNAT-2010: $\mathbf{P}=$ Peligro de extinción, $\mathbf{A}=$ Amenazada, $\mathbf{P r}=$ Sujeta a protección especial. Distribución: $\mathbf{B e}=$ Belice, Chis = Chiapas, $\mathbf{C}=$ Centroamerica, Dep = Depresión central de Chiapas, G = Guatemala, Hon = Honduras, Mich = Michoacán, Nic = Nicaragua, Oax = Oaxaca, $\mathbf{S}=$ Salvador, Tab $=$ Tabasco, Ver $=$ Veracruz. El guión representa los datos no disponibles. ${ }^{*}$ Nombres de los colectores en apéndice 2.

\begin{tabular}{lllll}
\hline Familia/Especie/ Colector & FC & FN & FE & MC \\
\hline
\end{tabular}

\section{TRACHEOPHYTA}

\section{LYCOPHYTA}

\section{Lycopodiaceae}

Huperzia cuernavacensis (Underw. \& F.E. Lloyd) Holub JAEJ663*

Huperzia taxifolia (Sw.) Trevis. JLPE6235

\section{Selaginellaceae}

Selaginella hoffmannii Hieron. JAEJ395

Selaginella pallescens (C. Presl) Spring. ACDI-5, DEB21538, JAEJ Y

JMM203, MAPF2099

Selaginella silvestris Aspl. DDLO13, HMC12

$\begin{array}{cccc}\mathrm{Hi} & \mathrm{Ep} & \mathrm{Fe} & 2 \\ \mathrm{Hi} & \mathrm{Ep} & \mathrm{Fe} & 10 \\ \mathrm{Hi} & \mathrm{Ru} & \mathrm{Fe} & 8 \\ \mathrm{Hi} & \mathrm{Au}, \mathrm{Ru} & \mathrm{Fe} & 7,10,11,12 \\ \mathrm{Hi}, \mathrm{Li} & \mathrm{Au} & - & 9\end{array}$

\section{MONILOPHYTA}

\section{Aspleniaceae}

Asplenium aff. pumilum Sw. JAEJ497

Asplenium cristatum Lam. TAS194

Asplenium barbaense Hieron. MAPF824

Asplenium praemorsum Sw. JAEJ463, 675

Asplenium pumilum Sw. DDLO11, HMC11, KGD2-BIS

Asplenium serra Langsd. \& Fischer MAPF825

Asplenium uniseriale Raddi MAPF829

Schaffneria nigripes Fée JAEJ411, MJP10

Athyriaceae

Woodsia mollis (Kaulf.) J. Sm. DEB51683

Blechnaceae

Blechnum glandulosum Kaulf. ex Link.MAPF827

Blechnum occidentale L. DEB33783, MAHM18

Dryopteridaceae

Dryopteris patula (Sw.) Underw. DEB39940

Elaphoglossum latifolium (Sw.) J. Sm. JAEJ459

Nephrolepis pectinata (Willd.) Schott DDLO8

Nephrolepis pendula (Raddi) J. Sm. JAEJ483

Nephrolepis undulata (Afzel. ex Sw.) J. Sm. DALG7, JAEJ456

Phanerophlebia gastonyi Yatsk. JAEJ417 Chis, Oax, Ver

Phanerophlebia juglandifolia (Humb. \& Bonpl. ex Willd.)

J. Sm. MAPF823, 823-bis

Tectaria heracleifolia (Willd.) Underw.HPU21, JAEJ651, MGPM1,

$$
\text { NDP, RKRM13, TAS200 }
$$

Tectaria transiens (C.V. Morton) A.R. Sm. JAEJ732

\section{Equisetaceae}

Equisetum myriochaetum Schltdl. \& Cham. JAEJ768

\section{Polypodiaceae}

Campyloneurum angustifolium (Sw.) Fée DEB \& ARS21559

Campyloneurum tenuipes Maxon MAPF828, MLMJ6, TAS193

\begin{tabular}{|c|c|c|c|}
\hline $\mathrm{Hi}$ & $\mathrm{Ru}$ & $\mathrm{Fe}$ & 11 \\
\hline $\mathrm{Hi}$ & $\mathrm{Au}$ & $\mathrm{Fe}$ & 2 \\
\hline $\mathrm{Hi}$ & Ep & $\mathrm{Fe}$ & 11 \\
\hline $\mathrm{Hi}$ & $\mathrm{Au}, \mathrm{Ep}$ & $\mathrm{Fe}$ & 2,10 \\
\hline $\mathrm{Hi}$ & $\mathrm{Au}$ & $\mathrm{Fe}$ & 9 \\
\hline $\mathrm{Hi}$ & Ep & $\mathrm{Fe}$ & 11 \\
\hline $\mathrm{Hi}$ & $\mathrm{Au}$ & $\mathrm{Fe}$ & 11 \\
\hline $\mathrm{Hi}$ & $\mathrm{Ru}$ & $\mathrm{Fe}$ & 8,9 \\
\hline $\mathrm{Hi}$ & $\mathrm{Au}$ & $\mathrm{Fe}$ & 7 \\
\hline $\mathrm{Hi}$ & $\mathrm{Au}$ & $\mathrm{Fe}$ & 11 \\
\hline $\mathrm{Hi}$ & $\mathrm{Au}$ & $\mathrm{Fe}$ & 2,5 \\
\hline $\mathrm{Hi}$ & $\mathrm{Au}$ & $\mathrm{Fe}$ & 9 \\
\hline $\mathrm{Hi}$ & $\mathrm{Au}$ & $\mathrm{Fe}$ & 10 \\
\hline $\mathrm{Hi}$ & $\mathrm{Au}$ & $\mathrm{Fe}$ & 9 \\
\hline $\mathrm{Hi}$ & $\mathrm{Ru}$ & $\mathrm{Fe}$ & 10 \\
\hline $\mathrm{Hi}$ & $\mathrm{Au}, \mathrm{Ru}$ & $\mathrm{Fe}$ & 9,10 \\
\hline $\mathrm{Hi}$ & $\mathrm{Au}$ & $\mathrm{Fe}$ & 8 \\
\hline $\mathrm{Hi}$ & $\mathrm{Au}$ & $\mathrm{Fe}$ & 11 \\
\hline $\mathrm{Hi}$ & $\mathrm{Au}$ & $\mathrm{Fe}$ & $2,4,5$ \\
\hline $\mathrm{Hi}$ & $\mathrm{Ru}$ & $\mathrm{Fe}$ & 4 \\
\hline $\mathrm{Hi}$ & $\mathrm{Au}$ & - & 1 \\
\hline $\mathrm{Hi}$ & Ep & $\mathrm{Fe}$ & 11 \\
\hline $\mathrm{Hi}$ & Ep & $\mathrm{Fe}$ & $2,9,11$ \\
\hline
\end{tabular}


Apéndice 1. Continuación

\begin{tabular}{|c|c|c|c|c|}
\hline Familia/Especie/ Colector & FC & FN & FE & MC \\
\hline Niphidium crassifolium (L.) Lellinger JAEJ664,MAPF822 & $\mathrm{Hi}$ & $\mathrm{Au}$ & $\mathrm{Fe}$ & 2,11 \\
\hline Pecluma alfredii (Rosenst.) M.G. Price MLMJ7 & $\mathrm{Hi}$ & $\mathrm{Au}$ & $\mathrm{Fe}$ & 9 \\
\hline Pecluma alfredii var. cupreolepis (A.M. Evans) A.R. Sm. JAEJ526 & $\mathrm{Hi}$ & $\mathrm{Au}$ & $\mathrm{Fe}$ & 11 \\
\hline Pecluma atra (A.M. Evans) M.G. Price AIG7, MAPF826 & $\mathrm{Hi}$ & Ep & $\mathrm{Fe}$ & 11 \\
\hline Pecluma ferruginea (M. Martens \& Galeotti) M.G. Price MAPF17 & $\mathrm{Hi}$ & $\mathrm{Au}$ & $\mathrm{Fe}$ & 9 \\
\hline Phlebodium areolatum (Humb. \& Bonpl. ex Willd.) J. Sm. JAEJ461, 672 & $\mathrm{Hi}$ & $\mathrm{Au}$ & $\mathrm{Fe}$ & 2,10 \\
\hline Pleopeltis crassinervata (Fée) T. Moore JAEJ674 & $\mathrm{Hi}$ & Ep & $\mathrm{Fe}$ & 2 \\
\hline Pleopeltis mexicana (Fée) Mickel \& Beitel JMPP20 & $\mathrm{Hi}$ & Ep & $\mathrm{Fe}$ & 10 \\
\hline Pleopeltis polylepis (Roem. ex Kunze) T. Moore JAEJ493 & $\mathrm{Hi}$ & Ep & $\mathrm{Fe}$ & 11 \\
\hline $\begin{array}{l}\text { Pleopeltis thyssanolepis (A. Braun ex Klotzsch) E.G. Andrews \& Windham } \\
\text { DEB37162, DEB \& ARS21547 }\end{array}$ & $\mathrm{Hi}$ & $\mathrm{Au}$ & Fe & 9,11 \\
\hline $\begin{array}{l}\text { Polypodium colpodes Kunze AMC2, AMC4, DEB37155, JAEJ399, } \\
\text { MMOs/n, RGOs/n }\end{array}$ & $\mathrm{Hi}$ & Ep, Ru & Fe & $8,9,11$ \\
\hline Polypodium cryptocarpon Fée DDLO15, KGD7, MJP13, MLMJ9 & $\mathrm{Hi}$ & $\mathrm{Au}, \mathrm{Ep}$ & $\mathrm{Fe}$ & 9 \\
\hline Polypodium falcaria Kunze JAEJ482 & $\mathrm{Hi}$ & $\mathrm{Au}$ & $\mathrm{Fe}$ & 10 \\
\hline $\begin{array}{l}\text { Polypodium furfuraceum Schltdl. \& Cham. AMNs/n, DEB\& ARS21597, } \\
\text { DEB\&ARS21590, JAEJ460, JC38, MMAN2, MME3, RGO1 }\end{array}$ & $\mathrm{Hi}$ & Ep & $\mathrm{Fe}$ & $1,10,11,12$ \\
\hline Polypodium hispidulum Bartlett DEB21593 & $\mathrm{Hi}$ & Ep & $\mathrm{Fe}$ & 11 \\
\hline Polypodium lindenianum Kunze JAEJ704 & $\mathrm{Hi}$ & Ep & $\mathrm{Fe}$ & 3 \\
\hline Polypodium plesiosorum Kunze HMC10, JAEJ409, 462, MGD5 & $\mathrm{Hi}$ & $\mathrm{Au}, \mathrm{Ep}$ & $\mathrm{Fe}$ & $9,8,10,11$ \\
\hline Polypodium pleurosorum Kunze ex Mett. CMZ1 & $\mathrm{Hi}$ & Ep & $\mathrm{Fe}$ & 11 \\
\hline Polypodium rhodopleuron Kunze IVS6 & $\mathrm{Hi}$ & Ep & $\mathrm{Fe}$ & 11 \\
\hline Polypodium thyssanolepis A. Braum ex Klotzsch JAEJ175, 484, JC37, VMM1 & $\mathrm{Hi}$ & $\mathrm{Au}, \mathrm{Ru}$ & $\mathrm{Fe}$ & $1,7,9,10$ \\
\hline Polypodium thyssanolepis var. thyssanolepis A. Braun ex Klotzsch MPMM5 & $\mathrm{Hi}$ & $\mathrm{Ru}$ & $\mathrm{Fe}$ & 11 \\
\hline Serpocaulon falcaria (kunze) A.R.Sm. AGG20 & $\mathrm{Hi}$ & $\mathrm{Au}$ & $\mathrm{Fe}$ & 10 \\
\hline Serpocaulon triseriale (Sw.) A.R. Sm. AGG19, JAEJ415, 458 A & $\mathrm{Hi}$ & $\mathrm{Ru}$ & $\mathrm{Fe}$ & 8,10 \\
\hline \multicolumn{5}{|l|}{ Pteridaceae } \\
\hline Adiantopsis radiata (L.) Fée JAEJ490 & $\mathrm{Hi}$ & $\mathrm{Au}$ & $\mathrm{Fe}$ & 11 \\
\hline Adiantum amplum C. Presl CAZM12 & $\mathrm{Hi}$ & $\mathrm{Au}$ & $\mathrm{Fe}$ & 5 \\
\hline Adiantum andicola Liebm. JAEJ491, MLMJ5 & $\mathrm{Hi}$ & $\mathrm{Au}$ & $\mathrm{Fe}$ & 9,11 \\
\hline Adiantum braunii Mett. ex Kunh DDLO12, DEB\&ARS21543, MPMM3 & $\mathrm{Hi}$ & $\mathrm{Au}, \mathrm{Ru}$ & $\mathrm{Fe}$ & 9,11 \\
\hline Adiantum capillus-veneris L. ERMA2, JAEJ596, LECV4, MGD9 & $\mathrm{Hi}$ & $\mathrm{Ru}$ & $\mathrm{Fe}$ & $1,9,11$ \\
\hline $\begin{array}{l}\text { Adiantum concinnum Humb. \& Bonpl. ex Willd. DALG1, DEB\&ARS21551, } \\
\text { GCLHI-3,JAEJ464, 542, PRZ6, REGR2, YN8 }\end{array}$ & $\mathrm{Hi}$ & $\mathrm{Au}$ & Fe & $9,10,11,12$ \\
\hline Adiantum lunulatum Burm. F. JAEJ408 & $\mathrm{Hi}$ & $\mathrm{Au}$ & $\mathrm{Fe}$ & 8 \\
\hline Adiantum pulverulentum L. GUR200, RMC638, RMC638 & $\mathrm{Hi}$ & $\mathrm{Au}$ & $\mathrm{Fe}$ & 3,8 \\
\hline Adiantum raddianum C. Presl DEB\&ARS21589 & $\mathrm{Hi}$ & $\mathrm{Au}$ & $\mathrm{Fe}$ & 11 \\
\hline Adiantum tenerum Sw. DEB\&ARS21587 & $\mathrm{Hi}$ & $\mathrm{Au}$ & $\mathrm{Fe}$ & 11 \\
\hline Adiantum trichochlaenum Mickel \& Beitel GUR234 & $\mathrm{Hi}$ & $\mathrm{Au}$ & $\mathrm{Fe}$ & 3 \\
\hline Adiantum tricholepis Fée AG17, ALA1, ARG \& et al.1191, MIGL1, REGR5 & $\mathrm{Hi}$ & $\mathrm{Ru}$ & - & $9,10,11$ \\
\hline Adiantum wilesianum Hook. TAS196 & $\mathrm{Hi}$ & $\mathrm{Au}$ & $\mathrm{Fe}$ & 2 \\
\hline Aleuritopteris farinosa (Forssk.) Fée CCG18 & $\mathrm{Hi}$ & $\mathrm{Ru}$ & - & 10 \\
\hline Cheilanthes angustifolia Kunth DEB51666, DEB52176 & $\mathrm{Hi}$ & $\mathrm{Au}$ & $\mathrm{Fe}$ & 7,8 \\
\hline Cheilanthes bonariensis (Willd.) Proctor MAPF2101 & $\mathrm{Hi}$ & $\mathrm{Au}$ & $\mathrm{Fe}$ & 12 \\
\hline Cheilanthes microphylla var. microphylla (Sw.) Sw. DEB51976 & $\mathrm{Hi}$ & $\mathrm{Au}$ & $\mathrm{Fe}$ & 8 \\
\hline Cheilanthes notholaenoides (Desv.) Maxon ex Weath. DEB33757 & $\mathrm{Hi}$ & $\mathrm{Ru}$ & $\mathrm{Fe}$ & 2 \\
\hline Cheiloplecton rigidum (Sw.) Fée & $\mathrm{Hi}$ & $\mathrm{Au}, \mathrm{Ru}$ & $\mathrm{Fe}$ & $7,8,9,11$ \\
\hline Cheiloplecton rigidum var. rigidum (Sw.) Fée JAEJ516 & $\mathrm{Hi}$ & $\mathrm{Ru}$ & $\mathrm{Fe}$ & 11 \\
\hline Notholaena candida (M. Martens \& Galeotti) Hook. DEB\&ARS21577, JC39 & $\mathrm{Hi}$ & $\mathrm{Ru}$ & $\mathrm{Fe}$ & 1,11 \\
\hline Pellaea atropurpurea (L.) Link DEB37179 & $\mathrm{Hi}$ & $\mathrm{Ru}$ & $\mathrm{Fe}$ & 9 \\
\hline
\end{tabular}


Apéndice 1. Continuación

\begin{tabular}{|c|c|c|c|c|}
\hline Familia/Especie/ Colector & FC & FN & FE & MC \\
\hline Pityrogramma calomelanos (L.) Link ACD6, RMC627 & $\mathrm{Hi}$ & $\mathrm{Au}$ & $\mathrm{Fe}$ & 8,10 \\
\hline Pityrogramma trifoliata (L.) R.M. Tryon DEB33810 & $\mathrm{Hi}$ & $\mathrm{Au}$ & $\mathrm{Fe}$ & 2 \\
\hline Pteris biaurita L. MAPF815, TAS202 & $\mathrm{Hi}$ & $\mathrm{Au}$ & $\mathrm{Fe}$ & 2,11 \\
\hline Pteris grandifolia L. ACDI-1, JAEJ657 & $\mathrm{Hi}$ & $\mathrm{Au}$ & $\mathrm{Fe}$ & 2,10 \\
\hline Pteris longifolia L. AGV15, DEB13851, HPU14, REGR14 & $\mathrm{Hi}$ & $\mathrm{Au}$ & $\mathrm{Fe}$ & 4,10 \\
\hline Pteris quadriaurita Retz. TAS201 & $\mathrm{Hi}$ & $\mathrm{Au}$ & $\mathrm{Fe}$ & 2 \\
\hline \multicolumn{5}{|l|}{ Schizaeaceae } \\
\hline Anemia adiantifolia (L.) Sw. DEB37219, DEB41535 & $\mathrm{Hi}$ & Еp & $\mathrm{Fe}$ & 9,11 \\
\hline Anemia hirsuta (L.) Sw. DEB51690 & $\mathrm{Hi}$ & $\mathrm{Ru}$ & $\mathrm{Fe}$ & 7 \\
\hline Anemia phyllitidis (L.) Sw. DDLO9 & $\mathrm{Hi}$ & $\mathrm{Ru}$ & $\mathrm{Fe}$ & 9 \\
\hline Anemia speciosa C. Presl DEB \& ARS21564, DEB27159, JAEJ492, 587 & $\mathrm{Hi}$ & $\mathrm{Au}$ & $\mathrm{Fe}$ & $1,8,11$ \\
\hline Lygodium venustum Sw. JLZ1 & $\mathrm{Hi}$ & $\mathrm{Au}$ & $\mathrm{Fe}$ & 11 \\
\hline \multicolumn{5}{|l|}{ Thelypteridaceae } \\
\hline Thelyperis cinerea (Sodiro) A.R. Sm. DDO10 & $\mathrm{Hi}$ & $\mathrm{Ru}$ & $\mathrm{Fe}$ & 9 \\
\hline Thelypteris hispidula (Decne)C.F. Reed AGG18 & $\mathrm{Hi}$ & $\mathrm{Au}$ & $\mathrm{Fe}$ & 10 \\
\hline Thelypteris kunthii (Desv.) C.V. Morton JAEJ597, 731 & $\mathrm{Hi}$ & $\mathrm{Au}, \mathrm{Ru}$ & $\mathrm{Fe}$ & 1 \\
\hline Thelypteris tetragona (Sw.) Small DEB9, MNFA2, TAS203 & $\mathrm{Hi}$ & $\mathrm{Au}$ & $\mathrm{Fe}$ & $2,9,10$ \\
\hline Thelypteris tuerckheimii (Donn. Sm.) C.F. Reed DEB\&EMC23535 & $\mathrm{Hi}$ & $\mathrm{Au}$ & - & 1 \\
\hline \multicolumn{5}{|l|}{ SPERMATOPHYTA } \\
\hline \multicolumn{5}{|l|}{ GIMNOSPERMAE } \\
\hline \multicolumn{5}{|l|}{ Cupresaceae } \\
\hline Juniperus gamboana Martínez RMC622 & Á & $\mathrm{Au}$ & $\mathrm{Fe}$ & 4 \\
\hline \multicolumn{5}{|l|}{ Pinaceae } \\
\hline Pinus oocarpa Schiede ex Schltdl., 1838. JAEJ381 & Á & $\mathrm{Au}$ & $\mathrm{Fr}$ & 10 \\
\hline \multicolumn{5}{|l|}{ Taxodiaceae } \\
\hline Taxodium mucronatum Ten. JAEJ436 & Á & $\mathrm{Au}$ & $\mathrm{Fr}$ & 10 \\
\hline \multicolumn{5}{|l|}{ Zamiaceae } \\
\hline Ceratozamia robusta Miq. JAEJ735, MAPF30, MAPF820 Chis, G, Ver, A & $\mathrm{Ar}$ & $\mathrm{Au}$ & $\mathrm{E}$ & $1,4,11$ \\
\hline Dioon sp. MEB s/n & $\mathrm{Ar}$ & $\mathrm{Au}$ & $\mathrm{E}$ & 5 \\
\hline \multicolumn{5}{|l|}{ ANGIOSPERMAE } \\
\hline \multicolumn{5}{|l|}{ LILIOPSIDA } \\
\hline \multicolumn{5}{|l|}{ Agavaceae } \\
\hline Agave angustifolia Haw. JAEJ332 & $\mathrm{Hi}$ & $\mathrm{Au}$ & $\mathrm{Fr}$ & 9 \\
\hline Agave grijalvensis Ullrich DEB9026, EE39, RRS500 Pr, Dep & $\mathrm{Hi}$ & $\mathrm{Au}$ & $\mathrm{Fl}, \mathrm{Fr}$ & 2,8 \\
\hline Agave seemanniana Jacobi JAEJ736 & $\mathrm{Hi}$ & $\mathrm{Au}$ & $\mathrm{Fr}$ & 4 \\
\hline Agave sisalana Perrine ex Engelm. AM, GUR \& EMS60 & $\mathrm{Hi}$ & $\mathrm{Au}$ & - & 2 \\
\hline Yucca guatemalensis Baker AMC7, KLA13 & $\mathrm{Hi}$ & $\mathrm{Au}$ & - & 1 \\
\hline \multicolumn{5}{|l|}{ Amaryllidaceae } \\
\hline Bomarea edulis (Tussac) Herb. JAEJ Y ALP296, LFMJ27 & $\mathrm{Li}$ & $\mathrm{Au}$ & $\mathrm{FI}$ & 8,10 \\
\hline Furcraea cabuya Trel. JAEJ364 & $\mathrm{Hi}$ & $\mathrm{Au}$ & $\mathrm{FI}$ & 9 \\
\hline Furcraea melanodonta Trel. OFC230 & $\mathrm{Hi}$ & $\mathrm{Au}$ & - & 8 \\
\hline Habranthus robustus Herb. ex Sweet GKGR23 & $\mathrm{Hi}$ & $\mathrm{Au}$ & - & 5 \\
\hline Hypoxis decumbens L. JAEJ778, OFS.1059 & $\mathrm{Hi}$ & $\mathrm{Au}$ & - & 1,6 \\
\hline Manfreda brachystachys (Cav.) Rose JAEJ403, 426 & $\mathrm{Hi}$ & $\mathrm{Au}$ & $\mathrm{FI}$ & 8 \\
\hline Manfreda variegata (Jacobi) Rose JAEJ418 & $\mathrm{Hi}$ & $\mathrm{Au}$ & - & 8 \\
\hline \multicolumn{5}{|l|}{ Araceae } \\
\hline Anthurium berriozabalense Matuda MAPF1706e Be, Chis, Oax & $\mathrm{Hi}$ & $\mathrm{Au}$ & $\mathrm{Fl}$ & 8 \\
\hline Anthurium clarinervium Matuda, 1954 JLC6 Chis & $\mathrm{Hi}$ & $\mathrm{Ru}$ & - & 4 \\
\hline
\end{tabular}


Apéndice 1. Continuación

\begin{tabular}{|c|c|c|c|c|}
\hline Familia/Especie/ Colector & FC & FN & FE & MC \\
\hline $\begin{array}{l}\text { Anthurium faustomirandae Pérez-Farrera \& Croat JAEJ602, } \\
\text { MAPF263,295, 1867A Chis }\end{array}$ & $\mathrm{Hi}$ & $\mathrm{Au}$ & - & 1,4 \\
\hline $\begin{array}{l}\text { Anthurium huixtlense Matuda JAEJ414, 588, 673, MAPF818, } \\
\text { OFS1068, TBMc375 }\end{array}$ & $\mathrm{Hi}$ & $\mathrm{Au}$ & - & $1,2,8,11$ \\
\hline Anthurium montanum Hemsl. GUR22 & $\mathrm{Hi}$ & & - & 2 \\
\hline Anthurium pentaphyllum (Aubl.) G. Don in Sweet CAPB27 & $\mathrm{Li}$ & $\mathrm{He}$ & - & 4 \\
\hline Anthurium scandens (Aubl.) Engl. CAPB26, DEB \& ARS21578, JAEJ385, 676 & $\mathrm{Hi}$ & Ep & - & $2,4,11$ \\
\hline $\begin{array}{l}\text { Anthurium schlechtendalii Kunth AM59, GUR167, 167a, GUR185, } \\
\text { JAEJ173, 217, 223, 661, MAPF2111, 804. }\end{array}$ & $\mathrm{Hi}$ & Ep, Au, Ru & $\mathrm{Fl}, \mathrm{Fr}$ & $2,3,5,7,12$ \\
\hline Anthurium schlechtendalii spp. schlechtendalii MPR\&AESs/n & $\mathrm{Hi}$ & $\mathrm{Ru}$ & - & 8 \\
\hline Anthurium seleri Engl. ALAC15, GUR24, JAEJ660, MAPF1706b Chis, G & $\mathrm{Hi}$ & $\mathrm{Au}$ & $\mathrm{Fl}, \mathrm{Fr}$ & $1,2,9$ \\
\hline Monstera deliciosa Liebm.JAEJ500, 592, 682 & $\mathrm{Hi}, \mathrm{Li}$ & $\mathrm{He}$ & $\mathrm{Fl}, \mathrm{Fr}$ & $1,2,11$ \\
\hline Monstera tuberculata Lundell JAEJ708 A & $\mathrm{Hi}$ & $\mathrm{He}$ & - & 3 \\
\hline Philodendron hederaceum (Jacq.) Schott JAEJ1090 & $\mathrm{Li}$ & $\mathrm{He}$ & - & 1 \\
\hline Philodendron jacquinii Schott JAEJ184 & $\mathrm{Li}$ & $\mathrm{Au}$ & $\mathrm{Fr}$ & 7 \\
\hline Philodendron radiatum var. radiatum MAPF262 & $\mathrm{Li}$ & $\mathrm{He}$ & - & - \\
\hline Philodendron warszewiczii K. Koch \& C.D. Bouché FMG5122 & $\mathrm{Li}$ & $\mathrm{He}$ & - & 1 \\
\hline Spathiphyllum brevirostre (Liebm.) Schott JAEJ652 & $\mathrm{Hi}$ & $\mathrm{Au}$ & $\mathrm{Fl}$ & 2 \\
\hline Spathiphyllum cochlearispathum (Liebm.) Engl JAEJ737 & $\mathrm{Hi}$ & $\mathrm{Ru}$ & $\mathrm{FI}$ & 4 \\
\hline Spathiphyllum phryniifolium Schott JAEJ763 & $\mathrm{Hi}$ & $\mathrm{Au}$ & $\mathrm{Fl}$ & 4 \\
\hline Syngonium podophyllum Schot JAEJ498, 605, 606, 667 & $\mathrm{Hi}$ & $\mathrm{Au}, \mathrm{He}$ & $\mathrm{FI}$ & $1,2,11$ \\
\hline \multicolumn{5}{|l|}{ Arecaceae } \\
\hline Acrocomia aculeata (Jacq.) Lodd. ex Mart. TBC\& DPH & $\operatorname{Ar}$ & $\mathrm{Au}$ & - & 2 \\
\hline $\begin{array}{l}\text { Brahea nitida André DEB,ER \& PH13397, DEB, PRH \& ER, JAEJ337, } \\
\text { MPMMs/n Pr }\end{array}$ & $\operatorname{Ar}$ & $\mathrm{Au}$ & - & 9,10 \\
\hline Chamaedorea arenbergiana H. Wendl. JAEJ653 A & $\operatorname{Ar}$ & $\mathrm{Au}$ & $\mathrm{Fr}$ & 2 \\
\hline Chamaedorea ernesti-augusti H. Wendl. JLC5, OFS1071 A & $\operatorname{Ar}$ & $\mathrm{Au}$ & $\mathrm{Fr}$ & 1,4 \\
\hline $\begin{array}{l}\text { Chamaedorea glaucifolia H. Wendl.CZCs/n, DEB\&FA48459, GDM\& } \\
\text { CZC1, GUR55, GUR55a, HQR3915, JAEJ\&ALP282, JAMA18, MAPF1706c, } \\
\text { 1706d P, Chis }\end{array}$ & $\operatorname{Ar}$ & $\mathrm{Au}$ & $\mathrm{Fl}, \mathrm{Fr}$ & $1,2,8,9,12$ \\
\hline Chamaedorea oblongata Mart. JAEJ760 & $\mathrm{Ab}$ & $\mathrm{Au}$ & - & 4 \\
\hline $\begin{array}{l}\text { Chamaedorea stolonifera H. Wendl. ex Hook. f. GUR54A, GUR54-bis, } \\
\text { JAEJ631, MAPFs/n, OFS1068 A, Chis, Gue }\end{array}$ & $\mathrm{Ab}$ & $\mathrm{Au}$ & $\mathrm{Fl}, \mathrm{Fr}$ & $1,2,3$ \\
\hline \multicolumn{5}{|l|}{ Bromeliaceae } \\
\hline Aechmea lueddmanniana (K. Koch) Mez JAEJ699, WAC26 & $\mathrm{Hi}$ & Ep, Ru & $\mathrm{Fl}$ & 3,4 \\
\hline Billbergia pallidiflora Liebm. GUR32, JAEJ776 & $\mathrm{Hi}$ & $\mathrm{Au}, \mathrm{Ep}$ & $\mathrm{Fr}$ & 2,6 \\
\hline Bromelia karatas L. GUR54, JAEJ595 & $\mathrm{Hi}$ & $\mathrm{Au}$ & $\mathrm{Fr}$ & 1,2 \\
\hline Bromelia pinguin L. AM, GUR \& EMS50 & $\mathrm{Hi}$ & $\mathrm{Au}$ & - & 2 \\
\hline Catopsis berteroniana (Schult. \& Schult. f.) Mez. GUR1 Pr & $\mathrm{Hi}$ & Ep & - & 2 \\
\hline Catopsis paniculata E. Morren GD29736 & $\mathrm{Hi}$ & Ep & - & 11 \\
\hline Catopsis sessiliflora (Ruiz \& Pav.) Mez MAPF831 & $\mathrm{Hi}$ & Ep & - & 11 \\
\hline Catopsis subulata L.B. Sm. DEB27170 & $\mathrm{Hi}$ & Ep & - & 8 \\
\hline Hechtia glomerata Zucc. DEB23916 & $\mathrm{Hi}$ & $\mathrm{Ru}$ & - & 2 \\
\hline Hechtia meziana L.B. Sm. JAEJ Y JMM191, JAEJ641 & $\mathrm{Hi}$ & $\mathrm{Au}, \mathrm{Ru}$ & $\mathrm{Fl}, \mathrm{Fr}$ & 2,7 \\
\hline $\begin{array}{l}\text { Pitcairnia breedlovei L.B. Sm. AM, GUR \& EMS45, } \\
\text { DEB27152,28173, JAEJ350 Chis }\end{array}$ & $\mathrm{Hi}$ & $\mathrm{Ru}$ & $\mathrm{Fl}, \mathrm{Fr}$ & $2,8,9$ \\
\hline Pitcairnia chiapensis Miranda DLA27, JAEJ182, 216 Chis & $\mathrm{Hi}$ & $\mathrm{Ru}$ & $\mathrm{Fl}$ & 5,7 \\
\hline Pitcairnia heterophylla (Lindl.) Beer. JAEJ677, NMM1250 & $\mathrm{Hi}$ & Ep & $\mathrm{Fl}$ & 1,2 \\
\hline Pitcairnia recurvata (Scheidw.) K. Koch WAC27 & $\mathrm{Hi}$ & $\mathrm{Ru}$ & - & 4 \\
\hline Tillandsia balbisiana Schult. f. GKGR24 & $\mathrm{Hi}$ & Ep & $\mathrm{Fl}$ & 5 \\
\hline
\end{tabular}


Apéndice 1. Continuación

\begin{tabular}{|c|c|c|c|c|}
\hline Familia/Especie/ Colector & FC & FN & FE & MC \\
\hline Tillandsia beutelspacheri Matuda NMM802 Chis & $\mathrm{Hi}$ & Ep & $\mathrm{Fl}$ & 5 \\
\hline Tillandsia capitata Griseb. AM, GUR \& EMS47 & $\mathrm{Hi}$ & $\mathrm{Au}$ & $\mathrm{Fl}$ & 2 \\
\hline Tillandsia capitata var guzmanioides L.B. Sm. JAEJ185 & $\mathrm{Hi}$ & $\mathrm{Ru}$ & $\mathrm{Fl}$ & 7 \\
\hline $\begin{array}{l}\text { Tillandsia caput-medusae E. Morren AM, GUR \& EMS46, } \\
\text { JAEJ325, 611, JGH1, NMM801 }\end{array}$ & $\mathrm{Hi}$ & Ep & $\mathrm{FI}$ & $2,4,5,9$ \\
\hline Tillandsia concolor L.B. Sm. VAGL20 A & $\mathrm{Hi}$ & Ep & $\mathrm{Fl}$ & 11 \\
\hline Tillandsia fasciculata Sw. AM, GUR \& EMS49, 53, JAEJ614 & $\mathrm{Hi}$ & Ep & $\mathrm{Fl}, \mathrm{Fr}$ & 2,8 \\
\hline Tillandsia filifolia Schltdl. \& Cham. RPLS/N & $\mathrm{Hi}$ & Ep & $\mathrm{Fl}$ & 7 \\
\hline $\begin{array}{l}\text { Tillandsia flabellata Baker AJUB26, AM, GUR \& EMS31, DEB27180, } \\
\text { 37215, GUR31, JAEJ Y ALP278, JAEJ390, 593, JLC10, JLOH35, OFS } \\
\text { y MAPF1000 }\end{array}$ & $\mathrm{Hi}$ & Ep & $\mathrm{Fl}, \mathrm{Fr}$ & $1,2,4,8,9,12$ \\
\hline Tillandsia guatemalensis L.B. Sm.GUR59, JAEJ615 & $\mathrm{Hi}$ & Ep & $\mathrm{Fl}, \mathrm{Fr}$ & 2 \\
\hline Tillandsia heterophylla E. Morren GUR61 Chis, Ver & $\mathrm{Hi}$ & Ep & $\mathrm{FI}$ & 2 \\
\hline Tillandsia ionantha var. vanhyningii M.B. Foster JAEJ655 & $\mathrm{Hi}$ & $\mathrm{Ru}$ & $\mathrm{Fl}$ & 2 \\
\hline $\begin{array}{l}\text { Tillandsia juncea (Ruiz \& Pav.) Poir. DEB49060, GUR51, JAEJ613, } \\
\text { MFC, AES, ARLF \&FRs/n. }\end{array}$ & $\mathrm{Hi}$ & Ep & $\mathrm{Fl}, \mathrm{Fr}$ & $1,2,9$ \\
\hline Tillandsia limbata Schltdl. FVH2968 & $\mathrm{Hi}$ & Ep & - & 4 \\
\hline Tillandsia polystachia (L.) L.DEB51526, GD29702, GUR53 & $\mathrm{Hi}$ & Ep & $\mathrm{Fr}$ & $2,7,11$ \\
\hline $\begin{array}{l}\text { Tillandsia schiedeana Steud. AM, GUR \& EMS48, JAEJ Y ALP307, } \\
\text { JAEJ612, 679, JM23 }\end{array}$ & $\mathrm{Hi}$ & Ep & $\mathrm{Fl}$ & $2,8,11$ \\
\hline Tillandsia seleriana Mez DEB9572, GKGR30, GUR4, RMC625 A & $\mathrm{Hi}$ & Ep & $\mathrm{Fl}, \mathrm{Fr}$ & $2,4,5,8$ \\
\hline Tillandsia streptophylla Scheidw. ex C. Morren EM151419 & $\mathrm{Hi}$ & Ep & - & 4 \\
\hline Tillandsia tricolor Schltdl. \& Cham.GUR49, 52 A & $\mathrm{Hi}$ & Ep & $\mathrm{Fl}$ & 2 \\
\hline \multicolumn{5}{|l|}{ Cannaceae } \\
\hline Canna edulis Ker Gawl. VAGL32 & $\mathrm{Hi}$ & $\mathrm{Au}$ & $\mathrm{FI}$ & 11 \\
\hline Canna indica L. MGRG101, LFMJ25 & $\mathrm{Ab}, \mathrm{Hi}$ & $\mathrm{Au}$ & $\mathrm{FI}$ & 2,10 \\
\hline \multicolumn{5}{|l|}{ Commelinaceae } \\
\hline $\begin{array}{l}\text { Callisia gentlei var. macdougallii (Miranda) D.R. Hunt DEB \& FSK s/n, } \\
\text { DEB14688, 27147, } 39961 \text { Chis }\end{array}$ & $\mathrm{Hi}$ & $\mathrm{Au}$ & $\mathrm{FI}$ & 8,9 \\
\hline Commelina diffusa Burm. F. JAEJ150, 209, MAPF2110 & $\mathrm{Hi}$ & $\mathrm{Au}$ & $\mathrm{FI}$ & 7,12 \\
\hline Commelina erecta L. DEB19984, MAPF2105 & $\mathrm{Hi}$ & $\mathrm{Au}$ & $\mathrm{FI}$ & $8,9,10,12$ \\
\hline $\begin{array}{l}\text { Tradescantia aff. deficiens Brandegee DEB69725,JAEJ \& ALP290, } \\
\text { JAEJ333, } 420\end{array}$ & $\mathrm{Hi}$ & $\mathrm{Au}$ & $\mathrm{FI}$ & 8,9 \\
\hline Tradescantia huehueteca (Standl. \& Steyerm.) A. Hunt MAP11 & $\mathrm{Hi}$ & $\mathrm{Au}$ & $\mathrm{Fl}$ & 1 \\
\hline Tradescantia zanonia (L.) Sw. JAEJ368, JLC7, OFS1069 & $\mathrm{Hi}$ & $\mathrm{Au}$ & $\mathrm{Fl}$ & 9 \\
\hline Tradescantia zebrina Heynh. DEB28167,37169, FMG7669, MAPF2106 & $\mathrm{Hi}$ & $\mathrm{Au}$ & $\mathrm{Fl}, \mathrm{Fr}$ & $1,9,12$ \\
\hline Tripogandra grandiflora (Donn. Sm.) Woodson DEB39951 Chis, C & $\mathrm{Hi}$ & $\mathrm{Au}$ & $\mathrm{Fl}$ & 9 \\
\hline Tripogandra montana Handlos DEB37177 & $\mathrm{Hi}$ & $\mathrm{Au}$ & $\mathrm{FI}$ & 9 \\
\hline Tripogandra serrulata (Vahl) Handlos AES \& MPRs/n & $\mathrm{Hi}$ & $\mathrm{Au}$ & $\mathrm{Fl}$ & 8 \\
\hline \multicolumn{5}{|l|}{ Cyperaceae } \\
\hline $\begin{array}{l}\text { Carex polystachya Sw. ex Wahlenb. DEB, PRH \& Ers/n, } \\
\text { DEB\& ARS21580, DEB13920 }\end{array}$ & $\mathrm{Hi}$ & $\mathrm{Au}$ & $\mathrm{FI}$ & 10,11 \\
\hline Carex scabrella Wahlenb. DEB, ER \& PH13396 & $\mathrm{Hi}$ & $\mathrm{Au}$ & $\mathrm{Fl}$ & 10 \\
\hline Cyperus articulatus L. JAEJ428 & $\mathrm{Hi}$ & $\mathrm{Au}$ & $\mathrm{Fl}$ & 8 \\
\hline Cyperus bipartitus Torr. GD29752 & $\mathrm{Hi}$ & $\mathrm{Au}$ & - & 11 \\
\hline Cyperus canus Presl. \& C. Presl. DEB33792 & $\mathrm{Hi}$ & $\mathrm{Au}$ & $\mathrm{FI}$ & 2 \\
\hline Cyperus hermaphroditus (Jacq.) Standl. ERB17 & $\mathrm{Hi}$ & $\mathrm{Au}$ & $\mathrm{Fl}$ & 10 \\
\hline Cyperus odoratus L. GD29753 & $\mathrm{Hi}$ & $\mathrm{Au}$ & $\mathrm{Fl}$ & 11 \\
\hline Cyperus tenerrimus J. PresI \& C. PresI JAEJ Y JMM193 & $\mathrm{Hi}$ & $\mathrm{Ru}$ & $\mathrm{Fl}$ & 7 \\
\hline Scleria lithosperma (L.) Sw. DEB39973 & $\mathrm{Hi}$ & $\mathrm{Au}$ & $\mathrm{Fl}$ & 9 \\
\hline
\end{tabular}


Apéndice 1. Continuación

\begin{tabular}{|c|c|c|c|c|}
\hline Familia/Especie/ Colector & FC & FN & FE & MC \\
\hline Dioscoreaceae & $\mathrm{Li}$ & $\mathrm{Au}$ & $\mathrm{Fl}, \mathrm{Fr}$ & 6,8 \\
\hline $\begin{array}{l}\text { Dioscorea carpomaculata O. Téllez \& B.G. Schub.FMG7912, } \\
\text { JAEJ Y JMM200, JAEJ120, } 321\end{array}$ & $\mathrm{Li}$ & $\mathrm{Au}$ & $\mathrm{Fl}, \mathrm{Fr}$ & $1,6,7,9$ \\
\hline Dioscorea composita Hemsl. JAEJ Y ALP312, JAEJ545 & $\mathrm{Li}$ & $\mathrm{Au}$ & $\mathrm{Fl}, \mathrm{Fr}$ & 8,12 \\
\hline Dioscorea convolvulacea Schltdl. \& Cham. DEB, ER \& PH13490,13545 & $\mathrm{Li}$ & $\mathrm{Au}$ & $\mathrm{Fl}$ & 10 \\
\hline Dioscorea cymosula Hemsl. TAS99 Chis, C & $\mathrm{Li}$ & $\mathrm{Au}$ & $\mathrm{Fl}$ & 7 \\
\hline Dioscorea densiflora HFs/n, OT, MSS\& EMS 7896, OT, MSS, EM, GD\& JD & $\mathrm{Li}$ & $\mathrm{Au}$ & $\mathrm{FI}$ & $1,10,11$ \\
\hline $\begin{array}{l}\text { Dioscorea floribunda M. Martens \& Galeotti DEB13925, 37258, 39957, } \\
\text { 51656, 52202, HF s/n, JAEJ \& ALP311 }\end{array}$ & $\mathrm{Li}$ & $\mathrm{Au}$ & $\mathrm{Fl}, \mathrm{Fr}$ & $7,8,9,10$ \\
\hline $\begin{array}{l}\text { Dioscorea gomez-pompae O. Téllez DEB, ER \& PH13524, DEB41513, } \\
\text { ARG, EMS, GUR \& AM161 Chis }\end{array}$ & $\mathrm{Li}$ & $\mathrm{Au}$ & $\mathrm{Fl}$ & 10,11 \\
\hline $\begin{array}{l}\text { Dioscorea liebmanii Uline HFs/n, JAEJ508, OT\&MSS\&EMS\&GD\&JD, } \\
\text { RTC, EC \& MH }\end{array}$ & $\mathrm{Li}$ & $\mathrm{Au}$ & $\mathrm{Fl}$ & 10,11 \\
\hline Dioscorea matagalpensis Uline JAEJ178 Chis, C & $\mathrm{Li}$ & $\mathrm{Au}$ & $\mathrm{Fl}$ & 7 \\
\hline Dioscorea nelsonii Uline ex R. Knuth HFs/n, JAEJ432 & $\mathrm{Li}$ & $\mathrm{Au}$ & $\mathrm{FI}$ & 8,10 \\
\hline Dioscorea pilosiuscula Bertero ex Spreng. JAEJ \& ALP301 & $\mathrm{Li}$ & $\mathrm{Au}$ & $\mathrm{Fl}$ & 8 \\
\hline Dioscorea sparsiflora Hemsl. FMG7912 & $\mathrm{Li}$ & $\mathrm{Au}$ & - & 10 \\
\hline Dioscorea subtomentosa Miranda DEB\&EMC34032, JAEJ212, DN5479 & $\mathrm{Li}$ & $\mathrm{Au}$ & $\mathrm{Fl}, \mathrm{Fr}$ & 3,7 \\
\hline Dioscorea sumiderensis B.G. Schub. \& O. Téllez C., DKC \& G, NMM798 Chis & $\mathrm{Li}$ & $\mathrm{Au}$ & $\mathrm{Fl}, \mathrm{Fr}$ & $5,8,10$ \\
\hline \multicolumn{5}{|l|}{ Heliconiaceae } \\
\hline Heliconia librata Griggs SAM23 & $\operatorname{Ar}$ & $\mathrm{Au}$ & $\mathrm{FI}$ & 5 \\
\hline Heliconia schiedeana Klotzsch JAEJ764 & $\mathrm{Hi}$ & $\mathrm{Au}$ & $\mathrm{Fl}$ & 4 \\
\hline \multicolumn{5}{|l|}{ Iridaceae } \\
\hline Cipura paludosa subsp. mexicana Ravenna OFS1058 & $\mathrm{Hi}$ & $\mathrm{Au}$ & $\mathrm{Fl}$ & 1 \\
\hline Sisyrinchium longispathum Conz. DEB27156 & $\mathrm{Hi}$ & $\mathrm{Au}$ & $\mathrm{FI}$ & 8 \\
\hline \multicolumn{5}{|l|}{ Lemnaceae } \\
\hline Lemna aequinoctialis Welw. DEB, ER \& PH8455 & $\mathrm{Hi}$ & Ac & - & 1 \\
\hline \multicolumn{5}{|l|}{ Liliaceae } \\
\hline Allium сера L. JHC1 & $\mathrm{Hi}$ & $\mathrm{Au}$ & - & 7 \\
\hline $\begin{array}{l}\text { Beaucarnea goldmanii Rose DEB9043, 28171, 39945, JAEJ344, } \\
\text { MAV1, OT\&LVR6682 A, Chis, C }\end{array}$ & Á, $\mathrm{Hi}$ & $\mathrm{Au}$ & $\mathrm{Fl}, \mathrm{Fr}$ & $1,2,6,7,9$ \\
\hline Echeandia breedlovei Cruden DEB41481 Chis, Oax & $\mathrm{Hi}$ & $\mathrm{Au}$ & - & - \\
\hline Echeandia chiapensis Cruden Chis, Oax & $\mathrm{Hi}$ & $\mathrm{Au}$ & - & - \\
\hline Echeandia parviflora Baker 52149, 27187, 37224 & $\mathrm{Hi}$ & $\mathrm{Au}$ & $\mathrm{Fl}$ & 8,9 \\
\hline Echeandia reflexa (Cav.) Rose EUVP19, JAEJ Y ALP264, JAEJ338, 380 & $\mathrm{Hi}$ & $\mathrm{Au}$ & $\mathrm{Fl}, \mathrm{Fr}$ & $8,9,10$ \\
\hline Hippeastrum puniceum (Lam.) Urb. JAEJ685 & $\mathrm{Hi}$ & $\mathrm{Au}$ & $\mathrm{Fl}$ & 1 \\
\hline $\begin{array}{l}\text { Schoenocaulon officinale (Schltdl. \& Cham.) A. Gray ex Benth. } \\
\text { AGP2581, OFC233 }\end{array}$ & $\mathrm{Hi}$ & $\mathrm{Au}$ & $\mathrm{Fl}$ & 8 \\
\hline \multicolumn{5}{|l|}{ Marantaceae } \\
\hline Calathea atropurpurea Matuda DEB \& MB68272 & $\mathrm{Hi}$ & $\mathrm{Au}$ & $\mathrm{Fl}$ & 5 \\
\hline Calathea coccinea Standl. \& Steyerm. CAPB28 & $\mathrm{Hi}$ & $\mathrm{Au}$ & $\mathrm{Fl}$ & 4 \\
\hline Maranta arundinacea L. DEB14681 & $\mathrm{Hi}$ & $\mathrm{Au}$ & $\mathrm{Fl}$ & 8 \\
\hline Maranta divaricata Roscoe JAEJ \& ALP249 & $\mathrm{Hi}$ & $\mathrm{Au}$ & $\mathrm{Fl}$ & 8 \\
\hline Maranta gibba Sm. DEB69737 & $\mathrm{Hi}$ & $\mathrm{Au}$ & $\mathrm{FI}$ & 9 \\
\hline \multicolumn{5}{|l|}{ Thalia geniculata L. VAGL31, AML5, ARG899, MLRP4 } \\
\hline \multicolumn{5}{|l|}{ Musaceae } \\
\hline Musa paradisiaca L. JAEJ722, RML918, 919 & $\operatorname{Ar}$ & $\mathrm{Au}$ & - & 4 \\
\hline \multicolumn{5}{|l|}{ Orchidaceae } \\
\hline Bletia purpurea (Lam.) DC.NMM795, JAEJ750 & $\mathrm{Hi}$ & $\mathrm{Ru}$ & $\mathrm{Fl}$ & 4,5 \\
\hline Brassavola cucullata (L.) R. Br. RLH7 & $\mathrm{Hi}$ & Ep & $\mathrm{Fl}$ & 1 \\
\hline
\end{tabular}


Apéndice 1. Continuación

\begin{tabular}{|c|c|c|c|c|}
\hline Familia/Especie/ Colector & FC & FN & FE & MC \\
\hline Cranichis apiculata Lindl. JAEJ525 & $\mathrm{Hi}$ & $\mathrm{Au}$ & $\mathrm{Fr}$ & 11 \\
\hline Cranichis cochleata Dressler DEB21546 Chis, G, Ver & $\mathrm{Hi}$ & - & $\mathrm{Fl}$ & 11 \\
\hline $\begin{array}{l}\text { Cyrtopodium macrobulbon (La Llave \& Lex.) G.A. Romero-Gonzalez } \\
\quad \text { \& Carnevali JAEJ101, 721, WAC25 }\end{array}$ & $\mathrm{Hi}$ & $\mathrm{Au}, \mathrm{Ru}$ & $\mathrm{Fl}, \mathrm{Fr}$ & $3,4,6$ \\
\hline Cyrtopodium punctatum (L.) Lindl. AM54 & $\mathrm{Hi}$ & $\mathrm{Au}$ & $\mathrm{Fl}$ & 2 \\
\hline Dichaea sp. JAEJ422 & $\mathrm{Hi}$ & Ep & $\mathrm{Fl}$ & 8 \\
\hline $\begin{array}{l}\text { Dichromanthus cinnabarinus subsp. cinnabarinus (La Llave \& Lex.) } \\
\text { Garay JAEJ \& ALP259 }\end{array}$ & $\mathrm{Hi}$ & $\mathrm{Au}$ & $\mathrm{FI}$ & 8 \\
\hline Dinema polybulbon (Sw.) Lindl. EMS, OT, MSS \& GDs/n & $\mathrm{Hi}$ & Ep & - & 11 \\
\hline Encyclia cf. diota (Lindl.) Schltr. RLH5 & $\mathrm{Hi}$ & Ep & - & 1 \\
\hline Encyclia bractescens (Lindl.) Hoehne AES, RR, BPG y CTs/n, NMM799 & $\mathrm{Hi}$ & Ep & $\mathrm{Fl}$ & 5 \\
\hline Epidendrum cardiochilum L.O. Williams JAEJ619 & $\mathrm{Hi}$ & Ep & $\mathrm{Fl}$ & 2 \\
\hline Epidendrum cardiphorum Schltr. JAEJ423 & $\mathrm{Hi}$ & Ep & $\mathrm{Fl}$ & 8 \\
\hline Epidendrum caroli Schltr. JWD35545 & $\mathrm{Hi}$ & Ep & - & 9 \\
\hline Epidendrum ciliare L. JAEJ786 & $\mathrm{Hi}$ & Ep & $\mathrm{Fl}$ & 6 \\
\hline Epidendrum clowesii Bateman ex Lindl. JAEJ747 & $\mathrm{Hi}$ & $\mathrm{Au}$ & $\mathrm{Fl}$ & 4 \\
\hline Epidendrum erectifolium Hágsater \& L. Sánchez S. JLPEs/n & $\mathrm{Hi}$ & Ep & - & - \\
\hline Epidendrum eustirum Ames, F.T. Hubb. \& C. Schweinf. GUR57 & $\mathrm{Hi}$ & Ep & - & 2 \\
\hline Epidendrum myrianthum Lindl. NMM796 & $\mathrm{Hi}$ & $\mathrm{Ru}$ & $\mathrm{FI}$ & 5 \\
\hline Epidendrum ramosum Jacq. DEB70026 & $\mathrm{Hi}$ & Ep & $\mathrm{FI}$ & 9 \\
\hline Epidendrum skutchii Ames, F.T. Hubb. \& C. Schweinf. EH6259, EMS8607 Pr & $\mathrm{Hi}$ & Ep & - & 11 \\
\hline Erycina pusilla (L.) N.H. Williams \& M.W. Chase JAEJ688 & $\mathrm{Hi}$ & Ep & $\mathrm{FI}$ & 3 \\
\hline Govenia alba A. Rich. \& Galeotti JAEJ401 & $\mathrm{Hi}$ & $\mathrm{Au}$ & $\mathrm{Fr}$ & 8 \\
\hline Govenia superba (La Llave \& Lex.) Lindl. JAEJ772 & $\mathrm{Hi}$ & $\mathrm{Au}$ & $\mathrm{FI}$ & 6 \\
\hline Guarianthe aurantiaca (Bateman ex Lindl.) Dressler \& W.E. Higgins ARG1455 & $\mathrm{Hi}$ & Ep & $\mathrm{Fl}$ & 1 \\
\hline Guarianthe skinneri (Bateman) Dressler \& W.E. Higgins JAEJ600 A, Chis, C & $\mathrm{Hi}$ & Ep & $\mathrm{Fl}$ & 1 \\
\hline Habenaria matudae Salazar AGP2583 & $\mathrm{Hi}$ & $\mathrm{Au}$ & $\mathrm{Fl}$ & 8 \\
\hline Habenaria quinqueseta (Michx.) Eaton JAEJ351 & $\mathrm{Hi}$ & $\mathrm{Au}$ & $\mathrm{FI}$ & 9 \\
\hline Isochilus carnosiflorus Lindl. JAEJ621 & $\mathrm{Hi}$ & Ep & $\mathrm{Fl}$ & 2 \\
\hline $\begin{array}{l}\text { Lycaste aromatica (Graham ex Hook.) Lindl. GUR23, NMM1251, } \\
\text { JAEJ617, } 698\end{array}$ & $\mathrm{Hi}$ & Ep & $\mathrm{Fl}$ & $1,2,3$ \\
\hline Lycaste consobrina Rchb. F. G.A. Salazar RL, GC \& GAS3665 & $\mathrm{Hi}$ & Ep & $\mathrm{FI}$ & 1 \\
\hline Maxillaria densa Lindl. JAEJ591 & $\mathrm{Hi}$ & Ep & $\mathrm{Fl}$ & 1 \\
\hline $\begin{array}{l}\text { Maxillaria variabilis Bateman ex Lindl. AM, GUR \& EMS42, } \\
\text { GUR42, JAEJ589, } 618\end{array}$ & $\mathrm{Hi}$ & Ep & $\mathrm{Fl}$ & 1,2 \\
\hline Mesadenus lucayanus (Britton) Schlecht NMM797 & $\mathrm{Hi}$ & $\mathrm{Au}$ & $\mathrm{Fl}$ & 5 \\
\hline Nageliella purpurea (Lindl.) L.O. Williams GKGR31 & $\mathrm{Hi}$ & Ep & $\mathrm{Fl}$ & 5 \\
\hline Nidema boothii (Lindl.) Schltr. GUR33 & $\mathrm{Hi}$ & Ep & $\mathrm{Fl}$ & 2 \\
\hline Notylia barkeri Lindl. JAEJ702, 703 & $\mathrm{Hi}$ & Ep & $\mathrm{Fl}$ & 3 \\
\hline Oeceoclades maculata (Lindl.) Lindl. JAEJ336 & $\mathrm{Hi}$ & $\mathrm{Au}$ & $\mathrm{Fl}$ & 9 \\
\hline Oncidium microchilum Bateman ex Lindl. NB1748 & $\mathrm{Hi}$ & Ep & - & 11 \\
\hline Oncidium ornithorhynchum Kunth DEB21562 & $\mathrm{Hi}$ & Ep & - & 11 \\
\hline Ornithocephalus tripterus Schlecht. OFS1064 & $\mathrm{Hi}$ & Ep & $\mathrm{Fl}$ & 1 \\
\hline Pleurothallis grobyi Bateman ex Lindl. RLD1387 & $\mathrm{Hi}$ & Ep & - & 7 \\
\hline Pleurothallis tubata (G. Lodd.) Steud. JAEJ620 & $\mathrm{Hi}$ & Ep & $\mathrm{FI}$ & 2 \\
\hline Polystachya cerea Lindl. GUR20 & $\mathrm{Hi}$ & Ep & $\mathrm{Fl}$ & 2 \\
\hline Ponera striata Lindl. JAEJ585, 697 & $\mathrm{Hi}$ & Ep & $\mathrm{Fl}$ & 1,3 \\
\hline Ponthieva parvula Schltr. JAEJ397 & $\mathrm{Hi}$ & $\mathrm{Au}$ & $\mathrm{Fl}$ & 8 \\
\hline $\begin{array}{l}\text { Prosthechea chacaoensis (Rchb. F.) W.E. Higgins AES, BPG, } \\
\text { MM, LP \& CTs/n }\end{array}$ & $\mathrm{Hi}$ & Ep & $\mathrm{FI}$ & 5 \\
\hline
\end{tabular}


Apéndice 1. Continuación

\begin{tabular}{|c|c|c|c|c|}
\hline Familia/Especie/ Colector & FC & FN & FE & MC \\
\hline $\begin{array}{l}\text { Prosthechea cochleata (L.) W.E. Higgins AES, RR, BPG y } \\
\text { CT, JAEJ590, 671,751 }\end{array}$ & $\mathrm{Hi}$ & Ep & $\mathrm{Fl}$ & $1,2,4,5$ \\
\hline Prosthechea livida (Lindl.) W.E. Higgins JAEJ406 & $\mathrm{Hi}$ & Ep & $\mathrm{Fl}$ & 8 \\
\hline $\begin{array}{l}\text { Prosthechea ochracea (Lindl.) W.E. Higgins AES, RR, BPG y CTs/n, GD, } \\
\text { MSS, OT, EMS \& JD, GKGR32, GUR50, NMM803, OFS1063 }\end{array}$ & $\mathrm{Hi}$ & $\mathrm{Au}, \mathrm{Ep}$ & $\mathrm{Fl}, \mathrm{Fr}$ & 5 \\
\hline Prosthechea radiata JAEJ680, 684, 695 & $\mathrm{Hi}$ & $\mathrm{Au}, \mathrm{Ep}$ & $\mathrm{Fl}$ & 2,3 \\
\hline $\begin{array}{l}\text { Pseudogoodyera pseudogoodyeroides (L.O. Williams) } \\
\quad \text { R. González \& Szlach. JAEJ633 }\end{array}$ & $\mathrm{Hi}$ & $\mathrm{Ru}$ & $\mathrm{Fr}$ & 2 \\
\hline Restrepiella ophiocephala (Lindl.) Garay \& Dunst. EMS5150 & $\mathrm{Hi}$ & Ep & - & 4 \\
\hline Rhyncholaelia glauca (Lindl.) Schltr. JAEJ616 & $\mathrm{Hi}$ & Ep & $\mathrm{Fl}$ & 2 \\
\hline Sarcoglottis cerina (Lindl.) P.N. Don JAEJ639 Pr & $\mathrm{Hi}$ & $\mathrm{Au}$ & $\mathrm{Fl}$ & 2 \\
\hline Sarcoglottis rosulata (Lindl.) P.N. Don GUR3 & $\mathrm{Hi}$ & $\mathrm{Au}$ & $\mathrm{Fl}, \mathrm{Fr}$ & 2 \\
\hline Sarcoglottis sceptrodes (Reichb.f.) Schltr. NMM1249 & $\mathrm{Hi}$ & $\mathrm{Au}$ & $\mathrm{Fl}$ & 1 \\
\hline Spiranthes hemichrea Lindl. EMS \& MSS \& ARGs/n & $\mathrm{Hi}$ & $\mathrm{Au}$ & - & 2 \\
\hline Stanhopea graveolens Lindl. EH1295 & $\mathrm{Hi}$ & Ep & - & 1 \\
\hline Stanhopea inodora G. Lodd. ex Lindl.EH1295 & $\mathrm{Hi}$ & Ep & - & 12 \\
\hline Stanhopea saccata Bateman MAPF1706-bis & $\mathrm{Hi}$ & Ep & - & 9 \\
\hline Stelis emarginata (Lindl.) Soto Arenas \& R. Solano GUR47 & $\mathrm{Hi}$ & Ep & $\mathrm{Fr}$ & 2 \\
\hline Stelis hymenantha Schltr. JAEJ757, OFS1065 & $\mathrm{Hi}$ & Ep & $\mathrm{Fl}$ & 1 \\
\hline Trichocentrum andreanum (Cogn.) R. Jiménez \& Carnevali JAEJ357, 424 & $\mathrm{Hi}$ & Ep & $\mathrm{Fl}$ & 8,9 \\
\hline $\begin{array}{l}\text { Trichocentrum carthagenense (Jacq.) M.W. Chase \& N.H. } \\
\text { Williams JAEJ514, } 361\end{array}$ & $\mathrm{Hi}$ & $\mathrm{Au}$ & $\mathrm{Fl}$ & 9,11 \\
\hline $\begin{array}{l}\text { Trichocentrum cebolleta (Jacq.) M.W. Chase \& N.H. } \\
\text { Williams AM51, JAEJ609 }\end{array}$ & $\mathrm{Hi}$ & Ep & $\mathrm{FI}$ & 2 \\
\hline Trichocentrum luridum ( Lindl.) M.W. Chase \& N.H. William RLH3 & $\mathrm{Hi}$ & Ep & $\mathrm{FI}$ & 1 \\
\hline $\begin{array}{l}\text { Trichocentrum microchilum (Bateman ex Lindley) M. W. } \\
\text { Chase et N.H. Williams OFS1054 }\end{array}$ & $\mathrm{Hi}$ & $\mathrm{Au}$ & $\mathrm{Fl}$ & 1 \\
\hline Trichopilia tortilis Lindl. JAEJ \& CAPB784 & $\mathrm{Hi}$ & Ep & $\mathrm{Fl}$ & 1 \\
\hline \multicolumn{5}{|l|}{ Poaceae } \\
\hline Andropogon fastigiatus Sw. GD29695 & $\mathrm{Hi}$ & $\mathrm{Au}$ & $\mathrm{Fl}$ & 11 \\
\hline Aristida gibbosa (Nees) Kunth DEB \& BB55490 & $\mathrm{Hi}$ & $\mathrm{Au}$ & $\mathrm{FI}$ & 11 \\
\hline Aristida ternipes Cav. GD29679 & $\mathrm{Hi}$ & $\mathrm{Au}$ & $\mathrm{FI}$ & 11 \\
\hline Bouteloua media (E. Fourn.) Gould \& Kapadia DEB, PRH \& Ers/n & $\mathrm{Hi}$ & $\mathrm{Au}$ & $\mathrm{Fl}$ & 10 \\
\hline Bouteloua triaena (Trin. ex Spreng.) Scribn. DEB \& FSKs/n, DEBs/n & $\mathrm{Hi}$ & $\mathrm{Au}$ & $\mathrm{FI}$ & 10 \\
\hline Cenchrus ciliaris L. JAEJ \& ALP262 & $\mathrm{Hi}$ & $\mathrm{Au}$ & $\mathrm{Fl}$ & 8 \\
\hline Cenchrus multiflorus J. Presl SDK74209 & $\mathrm{Hi}$ & $\mathrm{Au}$ & - & 10 \\
\hline Chloris ciliata Sw. DEB \& FSK & $\mathrm{Hi}$ & $\mathrm{Au}$ & $\mathrm{Fl}$ & 7 \\
\hline Chondrosum elatum (Reeder \& C. Reeder) Clayton DEBs/n & $\mathrm{Hi}$ & $\mathrm{Au}$ & $\mathrm{Fl}$ & 10 \\
\hline Chusquea sp. JAEJ518 & $\mathrm{Hi}$ & $\mathrm{Au}$ & E & 11 \\
\hline Eragrostis mexicana (Hornem.) Link DEB \& FSKs/n & $\mathrm{Hi}$ & $\mathrm{Au}$ & $\mathrm{FI}$ & 7 \\
\hline Eriochloa nelsonii Scribn. \& J.G. Sm. DEB \& FSKs/n & $\mathrm{Hi}$ & $\mathrm{Au}$ & $\mathrm{Fl}$ & 10 \\
\hline Hackelochloa granularis (L.) Kuntze DEB, ER \& PH13536 & $\mathrm{Hi}$ & $\mathrm{Au}$ & $\mathrm{Fl}$ & 10 \\
\hline $\begin{array}{l}\text { Heteropogon contortus (L.) P. Beauv. ex Roem. \& Schult. DEB, ER } \\
\quad \text { \& PH13382, DEB, PRH \& ER s/n, DEB \& B55485 }\end{array}$ & $\mathrm{Hi}$ & $\mathrm{Au}$ & $\mathrm{Fl}$ & 10,11 \\
\hline Hyparrhenia rufa (Nees) Stapf AM109, 110, JAEJ533 & $\mathrm{Hi}$ & $\mathrm{Au}$ & $\mathrm{Fl}$ & 2,12 \\
\hline Lasiacis aff. divaricata (L.) Hitcht. FHN87 & $\mathrm{Hi}$ & $\mathrm{Au}$ & - & 1 \\
\hline Lasiacis divaricata (L.) Hitchc. ERB12 & $\mathrm{Hi}$ & $\mathrm{Au}$ & $\mathrm{Fl}$ & 9 \\
\hline Lasiacis linearis Swallen JAEJ534 Chis, C & $\mathrm{Hi}$ & $\mathrm{Au}$ & $\mathrm{Fr}$ & 12 \\
\hline Lasiacis nigra Davidse EMSs/n, GD. Et al29711, JAEJ329, RMC640 & $\mathrm{Hi}$ & $\mathrm{Au}$ & $\mathrm{Fl}, \mathrm{Fr}$ & $8,9,11$ \\
\hline Lasiacis oaxacensis var. oaxacensis GD. Et al29758 & $\mathrm{Li}$ & $\mathrm{Au}$ & $\mathrm{Fl}$ & 11 \\
\hline
\end{tabular}


Apéndice 1. Continuación

\begin{tabular}{|c|c|c|c|c|}
\hline Familia/Especie/ Colector & FC & FN & FE & MC \\
\hline Lasiacis procerrima (Hack.) Hitchc. DEB13905 & $\mathrm{Hi}$ & $\mathrm{Au}$ & - & 10 \\
\hline Lasiacis rhizophora (E. Fourn.) Hitchc. GD. Et a/29728 & $\mathrm{Hi}$ & $\mathrm{Au}$ & $\mathrm{FI}$ & 11 \\
\hline Lasiacis ruscifolia (Kunth) Hitchc. DEB \& FSKs/n & $\mathrm{Hi}$ & $\mathrm{Au}$ & $\mathrm{FI}$ & 10 \\
\hline Leersia ligularis Trin. GD.29748 & $\mathrm{Hi}$ & $\mathrm{Au}$ & $\mathrm{Fl}$ & 11 \\
\hline Leptochloa virgata (L.) P. Beauv. GD. Et al29762 & $\mathrm{Hi}$ & $\mathrm{Au}$ & $\mathrm{FI}$ & 11 \\
\hline Melinis minutiflora P. Beauv JAEJ535 & $\mathrm{Hi}$ & $\mathrm{Au}$ & $\mathrm{Fl}$ & 12 \\
\hline Muhlenbergia robusta (E. Fourn.) Hitchc. AR215 & $\mathrm{Hi}$ & $\mathrm{Au}$ & - & 12 \\
\hline Muhlenbergia xanthodas Soderstr. AJZ43 & $\mathrm{Hi}$ & $\mathrm{Au}$ & - & 11 \\
\hline Oplismenus burmanni (Retz.) P. Beauv. GD. et a/29747 & $\mathrm{Hi}$ & $\mathrm{Au}$ & $\mathrm{Fl}$ & 11 \\
\hline $\begin{array}{l}\text { Otatea fimbriata Soderstrom AT,GM \& IP1009, GCR } \\
\text { \& HGs/n, JAEJ102, TRSs/n }\end{array}$ & $\mathrm{Ab}$ & $\mathrm{Au}$ & $\mathrm{Fl}, \mathrm{Fr}$ & $1,5,6,10$ \\
\hline Panicum arundinariae Trin. ex E. Fourn. DEB51676, JAEJ \& ALP291 & $\mathrm{Hi}$ & $\mathrm{Au}$ & $\mathrm{FI}$ & 7,8 \\
\hline Panicum cordovense E. Fourn. JAEJ \& ALP280 & $\mathrm{Hi}$ & $\mathrm{Au}$ & $\mathrm{FI}$ & 8 \\
\hline Panicum parcum Hitchc. \& Chase DEB, ER \& PH13492 & $\mathrm{Hi}$ & $\mathrm{Au}$ & $\mathrm{FI}$ & 10 \\
\hline $\begin{array}{l}\text { Panicum parviglume Hack. DEB51978, DEB, PRH \& Ers/n, DEB } \\
\text { \& BB55465, GD et al29727, GD.29765 }\end{array}$ & $\mathrm{Hi}$ & $\mathrm{Au}$ & $\mathrm{FI}$ & $8,10,11$ \\
\hline Paspalum botterii (E. Fourn.) Chase GD.29739, GD. et a/29757 & $\mathrm{Hi}$ & $\mathrm{Au}$ & $\mathrm{FI}$ & 11 \\
\hline Paspalum caespitosum Flüggé GD.29751 & $\mathrm{Hi}$ & $\mathrm{Au}$ & $\mathrm{FI}$ & 11 \\
\hline Paspalum cymbiforme E. Fourn. GD.29694 & $\mathrm{Hi}$ & $\mathrm{Au}$ & $\mathrm{FI}$ & 11 \\
\hline Paspalum paniculatum L. JAEJ \& ALP292 & $\mathrm{Hi}$ & $\mathrm{Au}$ & $\mathrm{FI}$ & 8 \\
\hline Pennisetum polystachion (L.) Schult. GD.29697 & $\mathrm{Hi}$ & $\mathrm{Au}$ & $\mathrm{FI}$ & 11 \\
\hline Pennisetum purpureum Schumach. JAEJ532 & $\mathrm{Hi}$ & $\mathrm{Au}$ & $\mathrm{Fl}$ & 12 \\
\hline Pereilema ciliatum E. Fourn. DEBs/n, RTCs/n, EC \& MH, RTC. et al.s/n & $\mathrm{Hi}$ & $\mathrm{Au}$ & $\mathrm{FI}$ & 10 \\
\hline Pereilema crinitum J. PresI GD. et a/29767 & $\mathrm{Hi}$ & $\mathrm{Au}$ & $\mathrm{Fl}$ & 11 \\
\hline Pharus lappulaceus Aubl. DEB70270, DEB70270a, JAEJ709 & $\mathrm{Hi}$ & $\mathrm{Au}$ & $\mathrm{Fl}, \mathrm{Fr}$ & $1,3,9$ \\
\hline Pharus parvifolius Nash FMG7910 & $\mathrm{Hi}$ & $\mathrm{Au}$ & $\mathrm{Fl}$ & 1 \\
\hline Rhipidocladum bartlettii (McClure) McClure HGs/n & $\mathrm{Ab}$ & $\mathrm{Au}$ & $\mathrm{Fl}$ & 7 \\
\hline Rhipidocladum pittieri (Hack.) McClure GM, AT \& IP1010 & $\mathrm{Li}$ & $\mathrm{Au}$ & $\mathrm{Fl}$ & 1 \\
\hline Rhynchelytrum repens (Willd.) C.E. Hubb. DLA32, JAEJ232, RMLs/n & $\mathrm{Hi}$ & $\mathrm{Au}$ & $\mathrm{Fl}, \mathrm{Fr}$ & 5,7 \\
\hline Schizachyrium sanguineum var. sanguineum GD.29674 & $\mathrm{Hi}$ & $\mathrm{Au}$ & $\mathrm{Fl}$ & 11 \\
\hline Setariopsis latiglumis (Vasey) Scribn. DEBs/n, DEB \& FSKs/n, RTC et al.s/n & $\mathrm{Hi}$ & $\mathrm{Au}$ & $\mathrm{Fl}$ & 10 \\
\hline Triniochloa stipoides (Kunth) Hitchc. AM109A & $\mathrm{Hi}$ & $\mathrm{Au}$ & $\mathrm{FI}$ & 2 \\
\hline \multicolumn{5}{|l|}{ Pontederiaceae } \\
\hline Eichhornia crassipes (Mart.) Solms. AMGND & $\mathrm{Hi}$ & Ac & $\mathrm{Fl}$ & 6 \\
\hline \multicolumn{5}{|l|}{ Smilacaceae } \\
\hline Smilax domingensis Willd. JAEJ638 & $\mathrm{Li}$ & $\mathrm{Au}$ & $\mathrm{Fr}$ & 2 \\
\hline Smilax mollis Humb. \& Bonpl. ex Willd. AM106, CMGH38 & $\mathrm{Li}$ & $\mathrm{Au}$ & $\mathrm{Fr}$ & 2,5 \\
\hline \multicolumn{5}{|l|}{ Typhaceae } \\
\hline Typha latifolia L. HPU74 & $\mathrm{Hi}$ & Ac & $\mathrm{FI}$ & 7 \\
\hline \multicolumn{5}{|l|}{ MAGNOLIOPSIDA } \\
\hline \multicolumn{5}{|l|}{ Acanthaceae } \\
\hline $\begin{array}{l}\text { Aphelandra scabra (Vahl) Sm. ARG957, DEB13879, D.E.B \& FSKs/n, } \\
\text { DLA24, EGA20, GD, MSS,OT,EMS \& DJ29721, GUR17, HB \& } \\
\text { MM15605, JAEJ103, 371, 509, JCM4, JICs/n, MAV2, MDRS6, } \\
\text { RTC6374, TPR1310, 1374, VMSL1114, YN9999, YNG\&GDM4 }\end{array}$ & $\mathrm{Ab}, \mathrm{Hi}$ & $\mathrm{Au}$ & $\mathrm{Fl}, \mathrm{Fr}$ & $\begin{array}{l}1,2,5,6,8 \\
9,10,11,12\end{array}$ \\
\hline $\begin{array}{l}\text { Barleria oenotheroides Dum. Cours. GD, MSS,OT,EMS } \\
\quad \text { \& DJ29716, JAEJ515, LFMJ15, JRV9 }\end{array}$ & $\mathrm{Hi}$ & $\mathrm{Au}$ & $\mathrm{Fl}$ & 10,11 \\
\hline Blechum grandiflorum Oerst. JAEJ694 & $\mathrm{Hi}$ & $\mathrm{Au}$ & $\mathrm{Fl}$ & 3 \\
\hline Blechum pyramidatum (Lam.) Urb. JJVJ25, RCC27 & $\mathrm{Hi}$ & $\mathrm{Au}$ & $\mathrm{Fl}$ & 5 \\
\hline
\end{tabular}


Apéndice 1. Continuación

\begin{tabular}{|c|c|c|c|c|}
\hline Familia/Especie/ Colector & FC & FN & FE & MC \\
\hline Chaetothylax leucanthus Leonard. GUR10, JAEJ686 & $\mathrm{Ab}$ & $\mathrm{Au}$ & $\mathrm{Fl}$ & 2 \\
\hline Dicliptera assurgens (L.) Juss. AM, GUR \& EMS99 & $\mathrm{Hi}$ & $\mathrm{Au}$ & $\mathrm{Fl}$ & 2 \\
\hline $\begin{array}{l}\text { Dicliptera sciadephora Donn. Sm. DEB49061, DEB9049, DTF } \\
\text { \& BB5026, ADS, JGP, CHPs/n, ADS,JGP,CHP975, GUR14, 14A, } \\
\text { 14-bis, MAMA1103, OT,EM \& MF6464 }\end{array}$ & $\mathrm{Ab}, \mathrm{Hi}$ & $\mathrm{Au}$ & $\mathrm{Fl}, \mathrm{Fr}$ & $1,2,3$ \\
\hline $\begin{array}{l}\text { Elytraria imbricata (Vahl) Pers. DCSas/n, DEB9047, DEB, ER } \\
\quad \text { \& PH13525, JAEJ196 }\end{array}$ & $\mathrm{Hi}$ & $\mathrm{Au}, \mathrm{Ru}$ & $\mathrm{Fl}, \mathrm{Fr}$ & $3,7,10$ \\
\hline Henrya insularis Nees. DCSs/n, ADS, JGP, CHPs/n, JJVJ23,OT6497 & $\mathrm{Ab}, \mathrm{Hi}$ & $\mathrm{Au}$ & $\mathrm{Fl}$ & $2,3,5$ \\
\hline Justicia brevifolia Lindau ex R. Knuth ADS974, JAEJ662 & $\mathrm{Hi}$ & $\mathrm{Au}$ & $\mathrm{Fl}$ & 2,3 \\
\hline Justicia campechiana Standl. ex Lundell LDP203 & $\mathrm{Hi}$ & $\mathrm{Au}$ & - & 6 \\
\hline Odontonema callistachyum (Schltdl. \& Cham.) Kuntze JAEJ707, JLC3 & $\mathrm{Hi}$ & $\mathrm{Au}$ & $\mathrm{Fl}$ & 3 \\
\hline Pseuderanthemum alatum (Nees) Radlk. JAEJ398 & $\mathrm{Hi}$ & $\mathrm{Au}$ & $\mathrm{Fl}$ & 8 \\
\hline Razisea spicata Oerst. JAEJ780 & $\mathrm{Hi}$ & $\mathrm{Au}$ & $\mathrm{Fl}$ & 1,6 \\
\hline $\begin{array}{l}\text { Ruellia breedlovei T.F. Daniel ALAC11, DEB9046, DTF5025, } \\
\text { JAEJ222, JJVJ30, LIDs/n Chis }\end{array}$ & $\mathrm{Hi}$ & $\mathrm{Au}, \mathrm{Ru}$ & $\mathrm{Fl}$ & $1,2,3,4,5$ \\
\hline Ruellia donnell-smithii Leonard JLNs/n & $\mathrm{Ab}$ & $\mathrm{Au}$ & $\mathrm{Fl}$ & 4 \\
\hline Ruellia hookeriana (Nees) Hemsl. DEB27264, JAEJ455 & $\mathrm{Hi}$ & $\mathrm{Au}$ & $\mathrm{Fl}$ & 8,10 \\
\hline Ruellia imbricata Forssk.JAEJ551 & $\mathrm{Hi}$ & $\mathrm{Au}$ & $\mathrm{Fl}$ & 12 \\
\hline $\begin{array}{l}\text { Ruellia inundata Kunth AM21, JAEJ138, 550, 690, MARR11, } \\
\text { JRV7, FDAL26 }\end{array}$ & $\mathrm{Ab}, \mathrm{Hi}$ & $\mathrm{Au}$ & $\mathrm{Fl}, \mathrm{Fr}$ & $\begin{array}{c}2,3,7 \\
10,11,12\end{array}$ \\
\hline $\begin{array}{l}\text { Ruellia jussieuoides Schltdl. \& Cham. AM, GUR \& EMS61, 61A, } \\
\text { 61-bis, 552, 689, 720, OHJL44 }\end{array}$ & $\mathrm{Hi}, \mathrm{Ab}$ & $\mathrm{Au}$ & $\mathrm{Fl}$ & $2,3,5,7,12$ \\
\hline Ruellia nudiflora (Engelm. \& A. Gray) Urb. DLA31 & $\mathrm{Hi}$ & $\mathrm{Au}$ & $\mathrm{Fl}$ & 5 \\
\hline Ruellia nudiflora var. nudiflora DEB \& FSKs/n & $\mathrm{Hi}$ & $\mathrm{Au}$ & $\mathrm{Fl}$ & 7 \\
\hline Ruellia pereducta Standl. ex Lundell MAMA1104, SZR11 & $\mathrm{Ab}$ & $\mathrm{Au}$ & $\mathrm{Fl}$ & 3,4 \\
\hline Tetramerium nervosum Nees AM, GUR \& EMS86, JAEJ538 JMM86 & $\mathrm{Hi}$ & $\mathrm{Au}$ & $\mathrm{Fl}, \mathrm{Fr}$ & $2,3,12$, \\
\hline Tetramerium tenuissimum Rose DCSs/n & $\mathrm{Hi}$ & $\mathrm{Au}$ & $\mathrm{FI}$ & 3 \\
\hline \multicolumn{5}{|l|}{ Amaranthaceae } \\
\hline Alternanthera laguroides (Standl.) Standl. EUVPs/n & $\mathrm{Hi}$ & $\mathrm{Au}$ & - & 10 \\
\hline Iresine arbuscula Uline \& W.L. Bray GUR159, JAEJ706 & Á, $\mathrm{Ab}$ & $\mathrm{Au}$ & $\mathrm{FI}$ & 3 \\
\hline Iresine calea (Ibáñez) Standl. HETR27, JAEJ136, JJVJ21 & $\mathrm{Ab}, \mathrm{Li}$ & $\mathrm{Au}$ & $\mathrm{FI}$ & 5,7 \\
\hline Iresine diffusa Humb. \& Bonpl. ex Willd. JAEJ512 & $\mathrm{Hi}$ & $\mathrm{Au}$ & $\mathrm{FI}$ & 11 \\
\hline Iresine nigra Uline \& W. L. Bray JGP580 & $\mathrm{Ab}$ & $\mathrm{Au}$ & $\mathrm{Fl}$ & 3 \\
\hline \multicolumn{5}{|l|}{ Anacardiaceae } \\
\hline Astronium graveolens Jacq. JAEJ76 A & Á & $\mathrm{Au}$ & $\mathrm{FI}$ & 6 \\
\hline Comocladia guatemalensis Donn. Sm. AM, GUR \& EMS34 Chis, G & Á & $\mathrm{Au}$ & $\mathrm{FI}$ & 2 \\
\hline Pistacia mexicana Kunth DEB9567 & Á & $\mathrm{Au}$ & $\mathrm{Fl}$ & 4 \\
\hline Rhus schiedeana Schltdl. DEB9569 & $\mathrm{Ab}$ & $\mathrm{Au}$ & $\mathrm{Fl}$ & 4 \\
\hline Rhus terebinthifolia Schltdl. \& Cham. JAEJ624, 775 & $\mathrm{Ab}, \mathrm{Li}$ & $\mathrm{Au}$ & $\mathrm{Fr}$ & 2,6 \\
\hline Spondias purpurea L. AMI, MO\& Ics/n, JAEJ125 & Á & $\mathrm{Au}$ & $\mathrm{Fr}$ & 6 \\
\hline \multicolumn{5}{|l|}{ Annonaceae } \\
\hline Annona diversifolia Saff. DEB37264 Chis, C & Á & $\mathrm{Au}$ & $\mathrm{Fr}$ & 9 \\
\hline Annona reticulata L. JAEJ78, 557 & Á & $\mathrm{Au}$ & $\mathrm{Fr}$ & 6,12 \\
\hline Guatteria amplifolia Triana \& Planch. JAEJ604, 758 & Á, $\mathrm{Ab}$ & $\mathrm{Au}$ & $\mathrm{Fl}, \mathrm{Fr}$ & 1,4 \\
\hline Malmea depressa (Baill.) R.E. Fr. GUR151-bis, GRZ105 & $\mathrm{Ab}$ & $\mathrm{Au}$ & - & 3 \\
\hline \multicolumn{5}{|l|}{ Apiaceae } \\
\hline $\begin{array}{l}\text { Donnellsmithia serrata (J.M. Coult. \& Rose) Mathias \& Constance } \\
\text { JAEJ \& ALP258 Chis, Oax }\end{array}$ & $\mathrm{Hi}$ & $\mathrm{Au}$ & $\mathrm{Fr}$ & 8 \\
\hline Eryngium carlinae F. Delaroche JMHJrs/n & $\mathrm{Hi}$ & $\mathrm{Au}$ & - & 5 \\
\hline
\end{tabular}


Apéndice 1. Continuación

\begin{tabular}{|c|c|c|c|c|}
\hline Familia/Especie/ Colector & FC & FN & FE & MC \\
\hline \multicolumn{5}{|l|}{ Apocynaceae } \\
\hline $\begin{array}{l}\text { Haplophyton cinereum (A. Rich.) Woodson DEB13850, } 20187 \text {, } \\
\text { DEB, ER \& PH13509, EE48, EUH48, JAEJ85, 172, JMM182 }\end{array}$ & $\mathrm{Hi}$ & $\mathrm{Au}$ & $\mathrm{Fl}, \mathrm{Fr}$ & $6,7,9,10$ \\
\hline Laubertia contorta (M. Martens \& Galeotti) Woodson DEB39960 & $\mathrm{Li}$ & $\mathrm{Au}$ & - & 9 \\
\hline Mandevilla aff. rosana (J. D. Smith) Woodson FMG7911 & $\mathrm{Li}$ & $\mathrm{Au}$ & - & 1 \\
\hline $\begin{array}{l}\text { Mandevilla acutiloba (A. DC.) Woodson DEB27268, DEB } \\
\quad \text { \& BB55471, FMG7911, JAEJ162 Chis, C }\end{array}$ & $\mathrm{Li}$ & $\mathrm{Au}$ & $\mathrm{Fl}$ & $7,8,10,11$ \\
\hline Mandevilla subsagittata (Ruiz \& Pav.) Woodson DEB37159, JAEJ335 & $\mathrm{Li}$ & $\mathrm{Au}$ & $\mathrm{FI}$ & 9 \\
\hline Mandevilla subsessilis (A. DC.) Woodson JLPE\&JIC\&CCC5756, JLPTs/n & $\mathrm{Li}$ & $\mathrm{Au}$ & $\mathrm{Fl}$ & 6 \\
\hline $\begin{array}{l}\text { Mandevilla tubiflora (M. Martens \& Galeotti) Woodson JAEJ346, } \\
\text { MF8579 Chis, C }\end{array}$ & $\mathrm{Li}$ & $\mathrm{Au}$ & $\mathrm{Fl}$ & 9,11 \\
\hline $\begin{array}{l}\text { Metastelma barbigerum Scheele var. liesnerianum (L. O. Williams) } \\
\text { Liede \& Meve. DEB14010 }\end{array}$ & $\mathrm{Li}$ & $\mathrm{Au}$ & - & 10 \\
\hline Metastelma brachymischum W. D. Stevens. DEB27183 Chis & $\mathrm{Li}$ & $\mathrm{Au}$ & - & 8 \\
\hline Metastelma longicoronatum (L.O. Williams) Liede DEB27183 & $\mathrm{Li}$ & $\mathrm{Au}$ & - & 8 \\
\hline Plumeria rubra L. DEB9611, L.D.E.B \& FSKs/n & Á & $\mathrm{Au}$ & $\mathrm{Fl}$ & 4 \\
\hline Prestonia mexicana A. DC. DEB69742 & $\mathrm{Li}$ & $\mathrm{Au}$ & $\mathrm{Fl}$ & 9 \\
\hline Rauvolfia tetraphylla L .AVTs/n, DEB37231 & $\mathrm{Ab}$ & $\mathrm{Au}$ & $\mathrm{Fl}$ & 9,10 \\
\hline Stemmadenia eubracteata Woodson DEB70252 & $\mathrm{Ab}$ & $\mathrm{Au}$ & $\mathrm{Fl}$ & 9 \\
\hline Stemmadenia grandiflora (Jacq.) Miers JAEJ158 & Á & $\mathrm{Au}$ & $\mathrm{Fl}$ & 7 \\
\hline Stemmadenia obovata K. Schum. CEMM19, DEB37248, JAEJ67, JAEJ392 & Á, $\mathrm{Ab}$ & $\mathrm{Au}$ & $\mathrm{Fl}$ & $6,8,9,10$ \\
\hline Tabernaemontana coronaria (Jacq.) Willd. UV29 & $\mathrm{Ab}$ & $\mathrm{Au}$ & $\mathrm{FI}$ & 1 \\
\hline Tabernaemontana amygdalifolia Jacq.JAEJ666 , JAEJ782 & Á & $\mathrm{Au}$ & $\mathrm{Fl}, \mathrm{Fr}$ & 2,6 \\
\hline $\begin{array}{l}\text { Thevetia ovata (Cav.) A. DC. AM, GUR \& EMS19, BRW29, DEB13868, } \\
\text { DEB \& EMC23457, DEB \& FSKs/n, MDRS9 }\end{array}$ & Á & $\mathrm{Au}$ & $\mathrm{Fl}, \mathrm{Fr}$ & $1,2,8,10$ \\
\hline $\begin{array}{l}\text { Tonduzia longifolia (A. DC.) Markgr. DEB30022, DEB, PRH } \\
\text { \& ER, FMG29, FMG7750, LDP537 }\end{array}$ & Á, $\mathrm{Ab}$ & $\mathrm{Au}$ & $\mathrm{FI}$ & $1,4,6,12$ \\
\hline Tonduzia macrantha Woodson DEB30022 Chis, G & $\mathrm{Ab}$ & $\mathrm{Au}$ & $\mathrm{FI}$ & 12 \\
\hline \multicolumn{5}{|l|}{ Araliaceae } \\
\hline Aralia humilis Cav. GLW17818 & $\mathrm{Ab}$ & $\mathrm{Au}$ & - & 8 \\
\hline Dendropanax arboreus (L.) Decne. \& Planch. DEB33761, 50652, RMC624 & Á & $\mathrm{Au}$ & - & $2,4,8$ \\
\hline $\begin{array}{l}\text { Oreopanax capitatus (Jacq.) Decne \& Planch. AES1723, GUR43, } \\
\text { 43-bis, GUR, EMS \& AM43 }\end{array}$ & Á & $\mathrm{Au}$ & $\mathrm{Fr}$ & 2,5 \\
\hline $\begin{array}{l}\text { Oreopanax peltatus Linden DEB9035, 9350, GUR60, 60A, 60-bis, GUR, } \\
\text { EMS \& AM60, JAEJ495, 583, JLOH33, MPMM6, VMM \& GVN7, VMs/n }\end{array}$ & Á & $\mathrm{Au}$ & $\mathrm{FI}$ & $1,2,5,11$ \\
\hline \multicolumn{5}{|l|}{ Aristolochiaceae } \\
\hline Aristolochia maxima Jacq. DEB37227 & $\mathrm{Li}$ & $\mathrm{Au}$ & $\mathrm{FI}$ & 9 \\
\hline \multicolumn{5}{|l|}{ Asclepiadaceae } \\
\hline Asclepias curassavica L. JMHJr10, SAM24, VWS1178 & $\mathrm{Hi}$ & $\mathrm{Au}$ & $\mathrm{Fl}, \mathrm{Fr}$ & $5,6,11$ \\
\hline Blepharodon mucronatum (Schltdl.) Decne. AESs/n, JAEJ502, OT6507 & $\mathrm{Li}$ & $\mathrm{Au}$ & $\mathrm{Fl}, \mathrm{Fr}$ & $2,8,11$ \\
\hline Gonolobus breedlovei L.O. Williams DEB14412 Chis & $\mathrm{Li}$ & $\mathrm{Au}$ & $\mathrm{Fl}$ & 7 \\
\hline Gonolobus uniflorus Kunth DEB \& FSKs/n, DEB10637, 51522 & $\mathrm{Li}$ & $\mathrm{Au}$ & $\mathrm{Fl}$ & 7 \\
\hline Marsdenia gualanensis Donn. Sm. JAEJ \& JMM204 & $\mathrm{Li}$ & $\mathrm{Au}$ & $\mathrm{Fl}, \mathrm{Fr}$ & 7,8 \\
\hline Marsdenia mexicana Decne. EMS20152, JAEJ \& ALP303 & $\mathrm{Li}$ & $\mathrm{Au}$ & - & 4 \\
\hline Matelea aspera (Mill.) W.D. Stevens DEB51686 & $\mathrm{Li}$ & $\mathrm{Au}$ & $\mathrm{Fl}$ & 7 \\
\hline Matelea inops Woodson JAEJ \& ALP260, 284, FMG7908, TAS102 Chis, Oax & $\mathrm{Li}$ & $\mathrm{Au}$ & $\mathrm{Fl}$ & 7,8 \\
\hline $\begin{array}{l}\text { Matelea quirosii (Standl.) Woodson ALH \& Ajs/n, DEB37187, } \\
\text { MHM966, RTC6336, 6342, } 6357\end{array}$ & $\mathrm{Li}$ & $\mathrm{Au}$ & $\mathrm{FI}$ & $7,9,10$ \\
\hline $\begin{array}{l}\text { Metastelma trichophyllum (L.O. Williams) W.D. } \\
\text { Stevens JCSN13336, RTC6334 }\end{array}$ & $\mathrm{Li}$ & $\mathrm{Au}$ & - & 10 \\
\hline
\end{tabular}


Inventario florístico del Parque Nacional Cañón del Sumidero, Chiapas, México

Apéndice 1. Continuación

Familia/Especie/ Colector

Asteraceae

Acmella radicans (Jacq.) R.K. Jansen JLPE, EAM \& YKM3095

Acourtia reticulata var. reticulata (Lag. ex D. Don) Reveal \& R.M.

King DEB \& FSK13906

Ageratina pichinchensis (Kunth) R.M. King \& H. Rob. JGP581

Ageratina pringlei (B.L. Rob. \& Greenm.) R.M. King \& H. Rob.

MAMA1111 Chis, G, Oax

Ageratina tomentella (Schrad.) R.M. King \& H. Rob. DEB \& FSK14019

Ageratum aff. rugosum J.M. Coult. JRV4

Ageratum conyzoides L. JAEJ326

Ageratum corymbosum Zuccagni DEB \& FSK13892

Aldama dentata La Llave DEB52153, JLPE3093

Baccharis trinervis Pers. JLNs/n, OT6459

Bidens alba var. radiata (Sch. Bip.) R.E. Ballard JAEJ446

Bidens pilosa L. DEB52196, ER, PRH \& DEB20130

Bidens squarrosa var. tereticaulis (DC.) Roseman DEB \& FSK41480

Brickellia diffusa (Vahl) A. Gray JLVR, JLPE \& ASTs/n

Brickellia oliganthes (Less.) A. Gray DEB \& BB55487

Brickellia paniculata (Mill.) B.L. Rob. OT6484, JLVR1170

Calea ternifolia Kunth DEB47044

Calea ternifolia var. ternifolia Kunth DEB \& FSK13907,

DEB \& FSK14512, DEB, PRH \& ER13542, LEU3336

Calea urticifolia (Mill.) DC. AM, GUR \& EMS103

Callistephus chinensis (L.) Nees EUVP2

Calyptocarpus wendlandii Sch. Bip. JAEJ \& ALP285

Carminatia tenuiflora DC. DEB, PRH \& ER13498

Chaptalia nutans (L.) Pol. DEB950, 39993

Chromolaena collina (DC.) R.M. King \& H. Rob. DEB47043, DEB \& FSK41495

Chromolaena quercetorum (L.O. Williams) R.M. King \& H. Rob. DEB \& ARS 21552, DEB \& FSK 13893, DEB \& FSK 41534, DEB, PRH \& ER13394

Conyza apurensis Kunth DEB52181

Cosmos caudatus Kunth DEB, PRH \& ER13373, JAEJ449

Cosmos crithmifolius Kunh DEB51663

Cosmos sulphureus Cav. DEB, PRH \& ER13547

Critonia hospitalis (B.L. Rob.) R. M. King \& H. Rob.

DEB21555, AJC10499 Chis, Hon, Ver

Critonia iltisii R.M. King \& H. Rob. DEB \& FSK, DEB49064 Chis, G

Dahlia coccinea Cav. DEB52192

Delilia biflora (L.) Kuntze DEB52169, DEB, PRH \& ER13494, JAEJ531

Desmanthodium perfoliatum Benth. DEB37157, 52180, JLPE2520,

JLPE \& CCC6234 Chis, Oax

Dyssodia porophyllum (Cav.) Cav. DEB13837

Dyssodia porophyllum var. cancellata (Cass.) Strother DEB \& FSK13500

Eupatoriastrum corvi (McVaugh) B.L. Turner DEB \& FSK41547JAEJ569 Chis

Eupatorium aschenbornianum S. SchauerFDAL24

Eupatorium crassirameum B.L. Rob. AM43, DEB \& FSK9573, MAMA1109, JLNs/n

Eupatorium galeottii B.L. Rob. JAEJ140

FC FN $\quad$ FE $\quad$ MC

$\mathrm{Hi}$

$\mathrm{Ab}$

$\begin{array}{llll}\mathrm{Ab} & \mathrm{Au} & \mathrm{Fl} & 3\end{array}$

$\mathrm{Ab}$

$\mathrm{Au}$

$\mathrm{Ab}$

$\mathrm{Ab}$

$\mathrm{Hi}$

$\mathrm{Hi}$

$\mathrm{Hi}$

$\mathrm{Ab}$

$\mathrm{Hi}$

$\mathrm{Hi}$

$\mathrm{Hi}$

$\mathrm{Hi}$

$\mathrm{Hi}$

$\mathrm{Hi}$

$A b$

$A b$

$\mathrm{Hi}$

$\mathrm{Hi}$

$\mathrm{Hi}$

$\mathrm{Hi}$

$\mathrm{Hi}$

$A b$

$\mathrm{Ab}$

$\mathrm{Au}$

$\mathrm{Au}$

$\mathrm{Au}$

$\mathrm{Au}$

$\mathrm{Au}$

$\mathrm{Au}$

$\mathrm{Au}$

$\mathrm{Au}$

$\mathrm{Au}$

$\mathrm{Au}$

$\mathrm{Au}$

$\mathrm{Au}$

$\mathrm{Au}$

$\mathrm{Au}$

$\mathrm{Au}$

$\mathrm{Au}$

$\mathrm{Au}$

$\mathrm{Au}$

$\mathrm{Au}$

$\mathrm{Au}$

$\mathrm{Hi}$

$\mathrm{Hi}$

$\mathrm{Au}$

$\mathrm{Au}$

$\mathrm{Hi}$

$\mathrm{Hi}$

Á

$\mathrm{Au}$

$\mathrm{Au}$

$\mathrm{Au}$

$\mathrm{Hi}$

$\mathrm{Hi}$

$\mathrm{Au}$

$\mathrm{Au}$

$\mathrm{Hi}$

$\mathrm{Au}$

$A b$

$\mathrm{Au}$

$A b$

$\mathrm{Ab}$

$\mathrm{Au}$

Au

$\mathrm{Au}$

$\mathrm{Au}$

$\mathrm{Hi}$

$\mathrm{Ab}$

$\mathrm{Au}$

$\mathrm{Ab}$

$\mathrm{Au}$

FI
FI

3

FI

FI $\quad 10$

$\mathrm{FI}$

$\mathrm{FI}$

$\mathrm{FI}$

$\mathrm{FI}$

$\mathrm{FI}$

$\mathrm{FI}$

$\mathrm{FI}$

$\mathrm{FI}$

12

MC

3

(1)

11

9

10

8,12

2,4

10

1,8

11

12

11

2,12

10

$7,10,11$

FI 2

FI 10

FI 8

FI 10

FI 9

FI 10, 11

FI 10,11

$\begin{array}{cc}\mathrm{FI} & 8 \\ \mathrm{FI} & 10 \\ \mathrm{FI} & 7 \\ \mathrm{FI} & 10 \\ \mathrm{FI} & 11\end{array}$

FI

$\mathrm{Fl}$

FI

$\mathrm{FI}$

$8,10,12$

$8,9,10$

FI $\quad 10$

FI 10

FI 11, 12

FI 10

FI 2, 3, 4 
Apéndice 1. Continuación

\begin{tabular}{|c|c|c|c|c|}
\hline Familia/Especie/ Colector & FC & FN & FE & MC \\
\hline $\begin{array}{l}\text { Eupatorium hospitale B.L. Rob. AJC10499, DEB \& ARS21555, DEB } \\
\quad \text { \& BB55476, DEB \& FA48473, DEB \& FSK13869, SBG \& SD2463 }\end{array}$ & $\mathrm{Ab}$ & $\mathrm{Au}$ & $\mathrm{Fl}$ & $1,10,11,12$ \\
\hline Eupatorium imitans B.L. Rob. JAEJ734 Chis, G, Oax & $\mathrm{Hi}$ & $\mathrm{Ru}$ & $\mathrm{Fl}$ & 4 \\
\hline Eupatorium leucocephalum Benth. JAEJ96,765, OHJL37 & Á, $\mathrm{Ab}, \mathrm{Hi}$ & $\mathrm{Au}$ & $\mathrm{Fl}$ & $4,5,6$ \\
\hline $\begin{array}{l}\text { Eupatorium odoratum L. AM57, BCS10, DEB \& FSK9040, } \\
\text { JAEJ554, JJVJ22, OHJL38 }\end{array}$ & $\mathrm{Ab}, \mathrm{Hi}$ & $\mathrm{Au}$ & $\mathrm{Fl}$ & $2,5,12$ \\
\hline Eupatorium selerianum B.L. Rob. SBG, ML, LAH, ATW \&KB & $\mathrm{Hi}$ & $\mathrm{Au}$ & $\mathrm{FI}$ & 1 \\
\hline Eupatorium shultzii Schnittsp. JGPs/n, JGP584, GUR12 & $\mathrm{Hi}$ & $\mathrm{Au}$ & $\mathrm{FI}$ & 2,3 \\
\hline Galeana pratensis (Kunth) Rydb. DEB52144 & $\mathrm{Hi}$ & $\mathrm{Au}$ & $\mathrm{Fl}$ & 8 \\
\hline Heterosperma pinnatum Cav. JAEJ441 & $\mathrm{Hi}$ & $\mathrm{Au}$ & $\mathrm{Fl}$ & 10 \\
\hline Hieracium gronovii L. DEB51664 & $\mathrm{Hi}$ & $\mathrm{Au}$ & $\mathrm{Fl}$ & 7 \\
\hline Hofmeisteria standleyi (S.F. Blake) R.M. King \& H. Rob.JAEJ733 & $\mathrm{Hi}$ & $\mathrm{Ru}$ & $\mathrm{Fl}$ & 4 \\
\hline Iostephane trilobata Hemsl. JAEJ476 & $\mathrm{Hi}$ & $\mathrm{Au}$ & $\mathrm{Fl}$ & 10 \\
\hline Isocarpha oppositifolia subsp. achyranthes (DC.) Borhidi DEB, PRH \& Ers/n & $\mathrm{Hi}$ & $\mathrm{Au}$ & $\mathrm{Fl}$ & 10 \\
\hline Koanophyllon ravenii R.M. King \& H. Rob.DEB \& FSKs/n, DEB \& FSKs/n & $\mathrm{Ab}$ & $\mathrm{Au}$ & $\mathrm{Fl}$ & 10,11 \\
\hline Koanophyllon solidaginoides (Kunth) R.M. King \& H. Rob. PAF \& EJLTTs/n & $\mathrm{Ab}$ & $\mathrm{Au}$ & $\mathrm{Fl}$ & 11 \\
\hline Lagascea helianthifolia Kunth AM, GUR \& EMS100, JAEJ529, TLW2376 & $\mathrm{Hi}$ & $\mathrm{Au}$ & $\mathrm{FI}$ & 2,12 \\
\hline Lagascea helianthifolia var. helianthifolia RML & $\mathrm{Hi}$ & $\mathrm{Au}$ & $\mathrm{FI}$ & 5 \\
\hline Lasianthaea fruticosa var. fruticosa (L.) K.M. Becker JAEJ157 & Á & $\mathrm{Au}$ & $\mathrm{Fl}$ & 7 \\
\hline Liabum andrieuxii (DC.) Benth. \& Hook. F. JAEJ367 & $\mathrm{Ab}$ & $\mathrm{Au}$ & $\mathrm{Fl}$ & 9 \\
\hline Liabum sublobatum B.L. Rob. JAEJ108, 580 & Á, $A b$ & $\mathrm{Au}$ & $\mathrm{FI}$ & 1,6 \\
\hline Melampodium divaricatum (Rich.) DC. DEB52154, DEB \& FSKs/n, RLH\&VAF & $\mathrm{Hi}$ & $\mathrm{Au}$ & $\mathrm{FI}$ & 8,9 \\
\hline Melampodium gracile Less. DEB \& FSKs/n, JBK \& CHP & $\mathrm{Hi}$ & $\mathrm{Au}$ & $\mathrm{FI}$ & 10 \\
\hline Melanthera nivea (L.) Small CSJs/n, DEB \& FSKs/n,DEB, PRH \& Ers/n, JLPEs/n & $\mathrm{Hi}$ & $\mathrm{Au}$ & $\mathrm{Fl}$ & $1,10,11$ \\
\hline Montanoa tomentosa Cerv.JAEJ558 & $\mathrm{Ab}$ & $\mathrm{Au}$ & $\mathrm{Fl}$ & 12 \\
\hline Neurolaena intermedia Rydb. JAEJ730 & $\mathrm{Hi}$ & $\mathrm{Au}$ & $\mathrm{FI}$ & 4 \\
\hline Otopappus scaber S. F. Blake AJC \& MSS s/n, DEB \& BB55468, JLPEs/n & $\mathrm{Li}$ & $\mathrm{Au}$ & $\mathrm{FI}$ & 10,11 \\
\hline $\begin{array}{l}\text { Otopappus verbesinoides Benth. AJC \& MSSs/n, DEB \& } \\
\text { BB55484, DEB \& FSKs/n }\end{array}$ & $\mathrm{Li}$ & $\mathrm{Au}$ & $\mathrm{FI}$ & 11 \\
\hline Parthenium hysterophorus L. JAEJ228, RMK2754 & $\mathrm{Hi}$ & $\mathrm{Au}$ & $\mathrm{Fl}$ & 6,7 \\
\hline Pectis prostrata Cav. DEB51657, 51994 & $\mathrm{Hi}$ & $\mathrm{Au}$ & $\mathrm{Fl}$ & 7,8 \\
\hline Pectis saturejaoides (Mill.) Sch. Bip. ex Seem. DEB \& FSKs/n & $\mathrm{Hi}$ & $\mathrm{Au}$ & $\mathrm{Fl}$ & 9 \\
\hline Pectis uniaristata var. holostemma A. Gray DEB52167 & $\mathrm{Hi}$ & $\mathrm{Au}$ & $\mathrm{Fl}$ & 8 \\
\hline Perityle microglossa var. microglossa Benth. GKGR33, JLPEs/n & $\mathrm{Hi}$ & $\mathrm{Au}$ & $\mathrm{FI}$ & 5,12 \\
\hline Perymenium grande Hemsl. JAEJ369 & $\mathrm{Ab}$ & $\mathrm{Au}$ & $\mathrm{Fl}$ & 8 \\
\hline $\begin{array}{l}\text { Perymenium grande var. nelsonii (B.L. Rob. \& Greenm.) J.J. Fay } \\
\text { DEB, PRH \& ER, JAEJ536, RLH\&VAFs/n }\end{array}$ & $\mathrm{Ab}, \mathrm{Hi}$ & $\mathrm{Au}$ & $\mathrm{FI}$ & $8,10,12$ \\
\hline $\begin{array}{l}\text { Philactis nelsonii (Greenm.) S.F. Blake DEB51971, 70044, DEB \& ARSs/n, } \\
\text { DSS \& GHs/n, ERB9, JAEJ \& ALP28, JLPEs/n, RLH\&VAFs/n }\end{array}$ & $\mathrm{Ab}$ & $\mathrm{Au}$ & $\mathrm{FI}$ & $8,9,10,11$ \\
\hline Pinaropappus spathulatus var. chiapensis McVaugh ER, PRH \& DEB20129 & $\mathrm{Hi}$ & $\mathrm{Au}$ & $\mathrm{Fl}$ & 1 \\
\hline Pittocaulon praecox (Cav.) H. Rob. \& Brettell JGPs/n & $\mathrm{Ab}$ & $\mathrm{Au}$ & $\mathrm{Fl}$ & 3 \\
\hline Pluchea carolinensis (Jacq.) G. Don JLNs/n, JP 4180 & $\mathrm{Ab}$ & $\mathrm{Au}$ & $\mathrm{Fl}$ & 2,4 \\
\hline Podachaenium eminens (Lag.) Sch. Bip.DEB9041, JGP \& JRWs/n, FMG7714 & $\mathrm{Ab}$ & $\mathrm{Au}$ & $\mathrm{Fl}$ & $1,2,3$ \\
\hline Pseudognaphalium elegans (Kunth) Kartesz JMHJrs/n & $\mathrm{Hi}$ & $\mathrm{Au}$ & $\mathrm{FI}$ & 9 \\
\hline Pseudokyrsteniopsis perpetiolata R.M. King \& H. Rob. PAF \& EJLTTs/n & $\mathrm{Ab}$ & $\mathrm{Au}$ & $\mathrm{Fl}$ & 11 \\
\hline Salmea orthocephala Standl. \& Steyerm. DEB \& FSKs/n & $\mathrm{Ab}$ & $\mathrm{Au}$ & $\mathrm{Fl}$ & 10 \\
\hline Sanvitalia procumbens Lam. DEB52146, JAEJ210 & $\mathrm{Hi}$ & $\mathrm{Au}$ & $\mathrm{Fl}$ & 7,8 \\
\hline Schkuhria pinnata (Lam.) Kuntze ex Thell. JAEJ520 & $\mathrm{Hi}$ & $\mathrm{Au}$ & $\mathrm{Fl}$ & 11 \\
\hline Senecio cf. oaxacanus Hemsl. DTR30 & $\mathrm{Ab}$ & $\mathrm{Au}$ & $\mathrm{Fl}$ & 5 \\
\hline Senecio chenopodioides Kunth AM, GUR \& EMS87 & $\mathrm{Hi}, \mathrm{Li}$ & $\mathrm{Au}$ & $\mathrm{FI}$ & $1,2,3,5,12$ \\
\hline
\end{tabular}


Inventario florístico del Parque Nacional Cañón del Sumidero, Chiapas, México

Apéndice 1. Continuación

\begin{tabular}{l} 
Familia/Especie/ Colector \\
\hline ARG, RH \&PJSs/n, JGPs/n, JAEJ537, MAMAs/n, OT, EMS \& FMs/n, \\
OT, PTL \& PMs/n, RCC29, SBG, ML, LAH, ATW \&KBs/n
\end{tabular}

Senecio deppeanus Hemsl JLNs/n, OT, PTL \& PMs/n

Simsia annectens var. grayi (Sch. Bip. ex S.F. Blake)

D.M. Spooner DEB \& JLS47040

Simsia foetida (Cav.) S.F. Blake JLVR

Simsia foetida var. foetida (Cav.) S.F. Blake AJC\&MSS s/n, DEB \&

FSKs/n, JLPEs/n, JLVR, JLPE \& ASTs/n, RLH\&VAFs/n

Sinclairia discolor Hook. \& Arn. JGP

Sinclairia glabra (Hemsl.) Rydb. ER, PRH \& DEB19763

Sinclairia polyantha (Klatt) Rydb. JAEJ700

Smallanthus maculatus (Cav.) H. Rob. DEB69721

Smallanthus oaxacanus (Sch. Bip. ex Klatt) H. Rob. DEB70029

Spilanthes ocymifolia (Lam.) A.H. Moore JAEJ530

Stevia elatior Kunth DEB51672

Stevia polycephala Bertol. JMHJr

Tagetes erecta L. JAEJ519, MELM128

Taraxacum officinale Weber ex F.H. Wigg.MELM151

Tithonia diversifolia (Hemsl.) A. Gray AM, GUR \& EMSNDs/n

Tithonia longiradiata (Bertol) S.F. Blake AM25, EMS, OT, MSS \& GDs/n

Tithonia rotundifolia (Mill.) S.F. Blake HMC5, JAEJ524, JCM2

Tithonia tubiformis (Jacq.) Cass. DEB \& FSKs/n, DEB \& FSKs/n

Tridax procumbens L. DLA30

Trixis chiapensis C.E. Anderson.DEB \& FSKs/n, DEB \& FSKs/n,

LEU \& Aps/n Chis, G

Trixis inula Crantz JGPs/n, JAEJ623

Verbesina breedlovei B.L. Turner AJCs/n, DEB \& BBs/n, DEB \& BB55467,

DEB \& FSKs/n, DEB \& JLS47042, s/n, JLPEs/n Chis, Gue, Mich

Verbesina crocata (Cav.) Less. DEB \& FA48474, EMS, OT, MSS, GD

\& JDs/n,JAEJ563, TLW, EJLTT \& JGPs/n

Verbesina gigantea Jacq. DEB \& FSKs/n, JAEJ156, 501, JLPEs/n

Verbesina hypargyrea B.L. Rob. \& Greenm. DEB47521, GUR2s/n, LEU

\& Aps/n, SBG, ML, LAH, ATW \&KBs/n

Verbesina neriifolia Hemsl. DEB \& FA48455, JLPEs/n Chis, Oax

Verbesina perymenioides Sch. Bip. ex Klatt JAEJ453, PAF \& EJLTT3247

Verbesina punctata B.L. Rob. \& Greenm. DEB \& FSK

Verbesina turbacensis Kunth JM6, JAEJ155

Vernonia canescens Kunth JAEJ749

Vernonia heydeana J.M. Coult. DEB \& KBT49599, SBG, ML, LAH, ATW \&KB

Vernonia patens Kunth. JAEJ135, JLNs/n

Viguiera aff. cordata (Hook. \& Arn.) D'Arcy PRZs/n

Viguiera cordata (Hook. \& Arn.) D'Arcy AJC\&M.MSSs/n, DEB

\& BB55473, DEB \& FSKs/n

Viguiera dentata (Cav.) Spreng. DEB \& BB55466

Viguiera dentata var. canescens (DC.) S.F. Blake EMS, OT, MSS \& GDs/n,

JLPE, \&EAM \&YKMs/n

Wedelia hispida var. ramosissima (Greenm.) K.M. Becker JAEJ323

Wedelia tehuantepecana B.L. Turner DEB, PRH \& Ers/n

Zexmenia aurantiaca Klatt DEB51980

Zinnia americana (Mill.) Olorode \& A.M. Torres EUH41

FC

FN

FE

MC

\begin{tabular}{|c|c|c|c|}
\hline$A b$ & $\mathrm{Au}$ & $\mathrm{Fl}$ & 3,4 \\
\hline $\mathrm{Hi}$ & $\mathrm{Au}$ & $\mathrm{FI}$ & 10 \\
\hline $\mathrm{Ab}$ & $\mathrm{Au}$ & $\mathrm{FI}$ & 12 \\
\hline $\mathrm{Hi}$ & $\mathrm{Au}$ & $\mathrm{FI}$ & 11 \\
\hline $\mathrm{Li}$ & Ep & $\mathrm{FI}$ & 3 \\
\hline $\mathrm{Ab}$ & $\mathrm{Au}$ & $\mathrm{Fl}$ & 1 \\
\hline $\mathrm{Hi}$ & Ep & $\mathrm{FI}$ & 3 \\
\hline $\mathrm{Ab}$ & $\mathrm{Au}$ & $\mathrm{FI}$ & 9 \\
\hline $\mathrm{Ab}$ & $\mathrm{Au}$ & $\mathrm{FI}$ & 9 \\
\hline $\mathrm{Hi}$ & $\mathrm{Au}$ & $\mathrm{Fl}$ & 12 \\
\hline $\mathrm{Hi}$ & $\mathrm{Au}$ & $\mathrm{FI}$ & 7 \\
\hline $\mathrm{Ab}$ & $\mathrm{Au}$ & $\mathrm{FI}$ & 9 \\
\hline $\mathrm{Hi}$ & $\mathrm{Au}$ & $\mathrm{FI}$ & 11,12 \\
\hline $\mathrm{Hi}$ & $\mathrm{Au}$ & $\mathrm{FI}$ & 6 \\
\hline $\mathrm{Hi}$ & $\mathrm{Au}$ & $\mathrm{Fl}$ & 10 \\
\hline $\mathrm{Hi}$ & $\mathrm{Au}$ & $\mathrm{FI}$ & 10,11 \\
\hline $\mathrm{Ab}, \mathrm{Hi}$ & $\mathrm{Au}$ & $\mathrm{FI}$ & 8,11 \\
\hline $\mathrm{Hi}$ & $\mathrm{Au}$ & $\mathrm{Fl}$ & 10,11 \\
\hline $\mathrm{Hi}$ & $\mathrm{Au}$ & $\mathrm{Fl}$ & 5 \\
\hline $\mathrm{Ab}$ & $\mathrm{Au}$ & $\mathrm{FI}$ & $1,10,11$ \\
\hline $\mathrm{Ab}$ & $\mathrm{Au}$ & $\mathrm{Fl}$ & 2,3 \\
\hline $\mathrm{Ab}$ & $\mathrm{Au}$ & $\mathrm{FI}$ & 10,11 \\
\hline $\mathrm{Hi}$ & $\mathrm{Au}$ & $\mathrm{FI}$ & 11,12 \\
\hline $\mathrm{Ab}$ & $\mathrm{Au}$ & $\mathrm{Fl}$ & $7,10,11$ \\
\hline $\mathrm{Ab}$ & $\mathrm{Au}$ & $\mathrm{FI}$ & $1,8,11$ \\
\hline $\mathrm{Ab}$ & $\mathrm{Au}$ & $\mathrm{FI}$ & 10,12 \\
\hline $\mathrm{Ab}$ & $\mathrm{Au}$ & $\mathrm{FI}$ & 10,11 \\
\hline $\mathrm{Hi}$ & $\mathrm{Au}$ & $\mathrm{FI}$ & 11 \\
\hline $\mathrm{Ab}$ & $\mathrm{Au}$ & $\mathrm{FI}$ & 7,11 \\
\hline $\mathrm{Ab}$ & $\mathrm{Au}$ & $\mathrm{FI}$ & 4 \\
\hline $\mathrm{Ab}$ & $\mathrm{Au}$ & $\mathrm{FI}$ & 1 \\
\hline$A b$ & $\mathrm{Au}$ & $\mathrm{FI}$ & 4,7 \\
\hline $\mathrm{Hi}$ & $\mathrm{Au}$ & $\mathrm{FI}$ & 11 \\
\hline $\mathrm{Hi}$ & $\mathrm{Au}$ & $\mathrm{FI}$ & 11,10 \\
\hline $\mathrm{Hi}$ & $\mathrm{Au}$ & $\mathrm{FI}$ & 11 \\
\hline $\mathrm{Hi}$ & $\mathrm{Au}$ & $\mathrm{FI}$ & 11,12 \\
\hline $\mathrm{Hi}$ & $\mathrm{Au}$ & $\mathrm{FI}$ & 9 \\
\hline $\mathrm{Hi}$ & $\mathrm{Au}$ & $\mathrm{FI}$ & 10 \\
\hline $\mathrm{Ab}$ & $\mathrm{Au}$ & $\mathrm{FI}$ & 8 \\
\hline $\mathrm{Ab}$ & $\mathrm{Au}$ & $\mathrm{FI}$ & 1 \\
\hline
\end{tabular}


Apéndice 1. Continuación

\begin{tabular}{|c|c|c|c|c|}
\hline Familia/Especie/ Colector & FC & FN & FE & MC \\
\hline Zinnia peruviana (L.) L. JAEJ451 & $\mathrm{Hi}$ & $\mathrm{Au}$ & $\mathrm{FI}$ & 10 \\
\hline \multicolumn{5}{|l|}{ Balanophoraceae } \\
\hline Helosis cayennensis (Sw.) Spreng. DEB27276 & $\mathrm{Hi}$ & Sa & - & 8 \\
\hline \multicolumn{5}{|l|}{ Balsaminaceae } \\
\hline Impatiens balsamina L. DEB14003 & $\mathrm{Hi}$ & $\mathrm{Au}$ & $\mathrm{FI}$ & 10 \\
\hline \multicolumn{5}{|l|}{ Begoniaceae } \\
\hline Begonia fusca Liebm. JAEJ761, JAEJ762 & $\mathrm{Hi}$ & $\mathrm{Au}$ & $\mathrm{Fl}$ & 4 \\
\hline Begonia heracleifolia Schltdl. \& Cham. JAEJ668 & $\mathrm{Hi}$ & $\mathrm{Au}$ & $\mathrm{Fl}$ & 2 \\
\hline Begonia oaxacana A. DC.LFMJ22 & $\mathrm{Hi}$ & $\mathrm{Ru}$ & $\mathrm{FI}$ & 10 \\
\hline Begonia philodendroides Ziesenh JAEJ413 Chis, Oax & $\mathrm{Hi}$ & $\mathrm{Au}$ & $\mathrm{Fr}$ & 8 \\
\hline Begonia pinetorum A. DC. GUR42, JAEJ404, 640 & $\mathrm{Hi}$ & $\mathrm{Ru}$ & $\mathrm{Fl}$ & $2,3,8$ \\
\hline $\begin{array}{l}\text { Begonia plebeja Liebm. ARG1198, DEB14004, 16001, DEB \& ARS21553, } \\
\text { DEB \& FSKs/n, GD29710, 8582, JG419 }\end{array}$ & $\mathrm{Hi}$ & $\mathrm{Au}$ & $\mathrm{FI}$ & $9,10,11$ \\
\hline Begonia sartorii Liebm.MAPF2113 & $\mathrm{Hi}$ & $\mathrm{Au}$ & $\mathrm{FI}$ & 12 \\
\hline \multicolumn{5}{|l|}{ Bignoniaceae } \\
\hline Adenocalymma inundatum Mart. ex DC. AES1698, JJVJ 19 & $\mathrm{Li}$ & $\mathrm{Au}$ & $\mathrm{FI}$ & 5 \\
\hline $\begin{array}{l}\text { Amphilophium paniculatum var molle (Schltdl. \& Cham.) Standl. AM, GUR } \\
\text { \& EMS89, DEB39926, GUR34, JAEJ111, OFC229 }\end{array}$ & $\mathrm{Li}$ & $\mathrm{Au}$ & $\mathrm{FI}$ & $2,6,8,9$, \\
\hline Amphitecna breedlovei A.H. Gentry DEB \& MB68270 Chis, G, S & Á & $\mathrm{Au}$ & $\mathrm{FI}$ & 5 \\
\hline Anemopaegma chrysanthum Dugand CRB263, GKGR25, JAEJ109, OHJL3 & $\mathrm{Li}$ & $\mathrm{Au}$ & $\mathrm{FI}$ & $5,6,10$ \\
\hline $\begin{array}{l}\text { Anemopaegma puberulum (Seibert) Miranda DEB9031, 24548, } \\
\text { ERB7, GUR29, WAC23 }\end{array}$ & $\mathrm{Li}$ & $\mathrm{Au}$ & $\mathrm{FI}$ & $2,4,5,9$ \\
\hline Arrabidaea corallina (Jacq.) Sandwith ARGs/n, DEB39956 & $\mathrm{Li}$ & $\mathrm{Au}$ & $\mathrm{FI}$ & 4,9 \\
\hline Arrabidaea erecta Miranda OGE7492 Chis & - & - & $\mathrm{F}$ & 5 \\
\hline Arrabidaea floribunda (Kunth) Loes. RTC, CM, \& GMs/n & $\mathrm{Li}$ & $\mathrm{Au}$ & $\mathrm{FI}$ & 4 \\
\hline $\begin{array}{l}\text { Arrabidaea mollissima (Kunth) Bureau \& K. Schum. GUR, } \\
\text { EMS \& AM23, JAEJ89 }\end{array}$ & $\mathrm{Ab}, \mathrm{Li}$ & $\mathrm{Au}$ & $\mathrm{Fl}$ & 2 \\
\hline Arrabidaea patellifera (Schltdl.) Sandwith DEB14693, JAEJ226 & $\mathrm{Li}$ & $\mathrm{Au}$ & $\mathrm{FI}$ & 7,8 \\
\hline Cydista aequinoctialis (L.) Miers JJVJ24 & $\mathrm{Li}$ & $\mathrm{Au}$ & $\mathrm{Fl}$ & 5 \\
\hline Cydista diversifolia (Kunth) Miers AM, GUR \& EMS24, 26 & $\mathrm{Li}$ & $\mathrm{Au}$ & $\mathrm{FI}$ & 2 \\
\hline Godmania aescutifolia (Kunth) Standl. JAEJ215 & Á & $\mathrm{Au}$ & $\mathrm{FI}$ & 7 \\
\hline Mansoa hymenaea (DC.) A.H. Gentry AM23, AM, GUR \& EMS23-bis & $\mathrm{Li}$ & $\mathrm{Au}$ & $\mathrm{FI}$ & 2 \\
\hline Melloa quadrivalvis (Jacq.) A.H. Gentry RTC, CM \& GMs/n & $\mathrm{Li}$ & $\mathrm{Au}$ & $\mathrm{FI}$ & 4 \\
\hline Pithecoctenium crucigerum (L.) A.H. Gentry JAEJ213 & $\mathrm{Li}$ & $\mathrm{Au}$ & $\mathrm{Fl}$ & 7 \\
\hline Podranea ricasoliana DEB \& EMC23558 & $\mathrm{Li}$ & $\mathrm{Au}$ & $\mathrm{FI}$ & 1 \\
\hline Pseudocalymma alliaceum (Lam.) Sandwith AM, GUR \& EMS3, JAEJ650 & $\mathrm{Li}$ & $\mathrm{Au}$ & $\mathrm{Fl}, \mathrm{Fr}$ & 2 \\
\hline Pyrostegia venusta (Ker Gawl.) Miers DEB \& EMC23552 & $\mathrm{Li}$ & $\mathrm{Au}$ & $\mathrm{Fl}$ & 1 \\
\hline Spathodea campanulata DEB20056 & Á & $\mathrm{Au}$ & $\mathrm{FI}$ & 10 \\
\hline Tabebuia chrysantha (Jacq.) G. Nicholson JAEJ648 A & Á & $\mathrm{Au}$ & $\mathrm{FI}$ & 2,3 \\
\hline Tabebuia chrysantha spp. chrysantha DN5488 & Á & $\mathrm{Au}$ & $\mathrm{FI}$ & 3 \\
\hline Tabebuia rosea (Bertol.) A. DC. JAEJ659 & Á & $\mathrm{Au}$ & $\mathrm{FI}$ & 2 \\
\hline $\begin{array}{l}\text { Tecoma stans (L.) Juss. ex. Kunth AM, GUR \& EMS8, DEB20031, } \\
\text { GRZ11, JAEJ152 }\end{array}$ & Á, Ab & $\mathrm{Au}$ & $\mathrm{FI}$ & $2,3,7,10$ \\
\hline \multicolumn{5}{|l|}{ Bixaceae } \\
\hline $\begin{array}{l}\text { Cochlospermum vitifolium (Willd.) Spreng. RMC628, JAEJ572, } \\
\text { JAEJ \& JMM205 }\end{array}$ & Á & $\mathrm{Au}$ & $\mathrm{Fl}, \mathrm{Fr}$ & $1,7,8$ \\
\hline \multicolumn{5}{|l|}{ Bombacaceae } \\
\hline Bernoullia flammea Oliver DEB9036, EMS, ARG, AM \& GUR & Á & $\mathrm{Au}$ & $\mathrm{Fl}$ & 2 \\
\hline $\begin{array}{l}\text { Ceiba aesculifolia (Kunth) Britten \& Baker f. AM,GUR \& EMS22, } \\
\text { JAEJ608, SAM29 }\end{array}$ & Á & $\mathrm{Au}$ & $\mathrm{Fl}, \mathrm{Fr}$ & 2,5 \\
\hline
\end{tabular}


Apéndice 1. Continuación

\begin{tabular}{|c|c|c|c|c|}
\hline Familia/Especie/ Colector & FC & FN & FE & MC \\
\hline Ceiba pentandra (L.) Gaertn. JAEJ430 & Á & $\mathrm{Au}$ & $\mathrm{E}$ & 8 \\
\hline Ochroma pyramidale (Cav. ex Lam.) Urb. JCP, LG \& LPZs/n & Á & $\mathrm{Au}$ & - & 7 \\
\hline Pseudobombax ellipticum (Kunth) Dugand JAEJ719 & Á & $\mathrm{Au}$ & - & 3 \\
\hline Quararibea funebris (La Llave) Vischer JAEJ710 & Á & $\mathrm{Au}$ & $\mathrm{E}$ & 3 \\
\hline Quararibea yunckeri subsp. sessiliflora Miranda ex W.S. Alverson JCP1998 & Á & $\mathrm{Au}$ & $\mathrm{Fl}$ & 8 \\
\hline \multicolumn{5}{|l|}{ Boraginaceae } \\
\hline Bourreria huanita (Lex.) Hemsl. DEB14406 & Á & $\mathrm{Au}$ & - & 7 \\
\hline $\begin{array}{l}\text { Cordia alliodora (Ruiz \& Pav.) Oken DEB13885, DEB \& FSKs/n, } \\
\text { ER, PRH20120, FDAL29 }\end{array}$ & Á & $\mathrm{Au}$ & $\mathrm{FI}$ & 1,10 \\
\hline Cordia dentata Poir. LFMJ13, DEB20069, JAEJ \& JMM195, LAJV4 & Á & $\mathrm{Au}, \mathrm{Ru}$ & $\mathrm{Fl}, \mathrm{Fr}$ & $6,7,10$ \\
\hline Cordia dodecandra A. DC. DEB \& EMC34037, ECQ3, MNMs/n, UV11 & Á & $\mathrm{Au}$ & $\mathrm{Fl}, \mathrm{Fr}$ & $3,5,7$ \\
\hline Cordia foliosa M. Martens \& Galeotti DEB20105, 37196, 52184 & Á, $A b$ & $\mathrm{Au}$ & $\mathrm{Fl}$ & $8,9,10$ \\
\hline $\begin{array}{l}\text { Cordia spinescens L. DEB10668, 37201, 37246, 51680, DEB } \\
\quad \text { \& FSKs/n, JAEJ \& ALP298 }\end{array}$ & $\mathrm{Ab}$ & $\mathrm{Au}$ & $\mathrm{Fl}$ & $7,8,9$ \\
\hline Ehretia latifolia DC. FMG7752 & $\mathrm{Ab}$ & $\mathrm{Au}$ & - & 1 \\
\hline Ehretia tinifolia L. DEB \& EMC34039 & Á & $\mathrm{Au}$ & - & 3 \\
\hline Heliotropium angiospermum Murray DEB37244 & $\mathrm{Hi}$ & $\mathrm{Au}$ & $\mathrm{Fl}$ & 9 \\
\hline Heliotropium fallax I.M. Johnst. DEB27148 & $\mathrm{Ab}$ & $\mathrm{Au}$ & $\mathrm{Fl}$ & 8 \\
\hline Heliotropium fruticosum L. DEB20086 & $\mathrm{Hi}$ & $\mathrm{Au}$ & $\mathrm{Fl}$ & 10 \\
\hline Heliotropium indicum L. JAEJ148 & $\mathrm{Ab}$ & $\mathrm{Au}$ & $\mathrm{Fl}$ & 7 \\
\hline Heliotropium pringlei B.L. Rob. DEB39982, DEB \& FSKs/n & $\mathrm{Hi}$ & $\mathrm{Au}$ & $\mathrm{Fl}$ & 9 \\
\hline Heliotropium rufipilum (Benth.) I.M. Johnst. DEB41553 & $\mathrm{Hi}$ & $\mathrm{Au}$ & $\mathrm{Fl}$ & 11 \\
\hline Heliotropium ternatum Vahl WCH, JWY \& WWS3452 & $\mathrm{Ab}$ & $\mathrm{Au}$ & $\mathrm{Fl}$ & 5 \\
\hline $\begin{array}{l}\text { Tournefortia densiflora M. Martens \& Galeotti ARG167, } \\
\text { DEB37267, EMS22319 }\end{array}$ & $\mathrm{Ab}$ & $\mathrm{Au}$ & $\mathrm{Fl}, \mathrm{Fr}$ & $2,9,12$ \\
\hline Tournefortia glabra L. DEB37238, 51515, 51961 & $\mathrm{Ab}$ & $\mathrm{Au}$ & $\mathrm{FI}$ & $7,8,9$ \\
\hline Tournefortia hirsutissima L. DEB51513 & $\mathrm{Ab}$ & $\mathrm{Au}$ & $\mathrm{Fl}$ & 7 \\
\hline Tournefortia petiolaris DC. DEB41548 & $\mathrm{Ab}$ & $\mathrm{Au}$ & $\mathrm{Fl}$ & 11 \\
\hline Tournefortia volubilis L. JAEJ71 & $\mathrm{Li}$ & $\mathrm{Au}$ & $\mathrm{Fl}$ & 6 \\
\hline \multicolumn{5}{|l|}{ Brassicaceae } \\
\hline Lepidium virginicum var. centrali-americanum Thell. DEB39988 & $\mathrm{Hi}$ & $\mathrm{Au}$ & - & 9 \\
\hline \multicolumn{5}{|l|}{ Burseraceae } \\
\hline Bursera arborea (Rose) L. Riley CT, BPG,MM, LP \& AESs/n A & Á & $\mathrm{Au}$ & - & 5 \\
\hline Bursera ariensis (Kunth) McVaugh \& Rzed. AM, GUR \& EMS35 & Á & $\mathrm{Au}$ & $\mathrm{Fl}$ & 2 \\
\hline $\begin{array}{l}\text { Bursera bipinnata (DC.) Engl. DEB \& RFTs/n, JAEJ \& ALP250, } \\
\text { LFMJ12, RMcVs/n, RMC626 }\end{array}$ & Á & $\mathrm{Au}$ & $\mathrm{Fr}$ & 8,10 \\
\hline $\begin{array}{l}\text { Bursera excelsa (Kunth) Engl. JAEJ167 AMT \& MCMLs/n, DEB37265, } \\
\text { 37266, 39962, DEB \& ARSs/n, DEB \& ARS21556, DEB, ER \& PH13533, } \\
\text { DEB, PRH \& Ers/n, JAEJ94, LFMJ10, MDRS14, RFT \& Els/n, RPM40 }\end{array}$ & $\mathrm{Ab}$ & $\mathrm{Au}$ & Fr & $\begin{array}{c}5,6,7,9 \\
10,11\end{array}$ \\
\hline $\begin{array}{l}\text { Bursera longipes (Rose) Standl. CATM185, 186, 187, CT, AES, MM, } \\
\qquad \text { LP \& BPGs/n, CT, BPG, MM, LP, AESs/n }\end{array}$ & Á, $A b$ & $\mathrm{Au}$ & - & 5 \\
\hline Bursera schlechtendalii Engl. DEB39934, DN5556 & $\mathrm{Ab}$ & $\mathrm{Au}$ & $\mathrm{Fl}$ & $3,5,7,9$ \\
\hline $\begin{array}{l}\text { Bursera simaruba (L.) Sarg. AMT \& MCMLs/n, ARG881, 884, 938, CT, } \\
\text { AES, MM, LP \& BPGs/n, DEB20073, JAEJ154, WCH, JWY \& WWSs/n }\end{array}$ & Á & $\mathrm{Au}$ & $\mathrm{Fl}, \mathrm{Fr}$ & $5,9,11$ \\
\hline Bursera tomentosa (Jacq.) Triana \& Planch.ARG909, EUVP9 & Á & $\mathrm{Au}$ & $\mathrm{Fr}$ & 9,10 \\
\hline Protium copal (Schltdl. \& Cham.) Engl. JAEJ394 & Á & $\mathrm{Au}$ & $\mathrm{E}$ & 8 \\
\hline \multicolumn{5}{|l|}{ Cactaceae } \\
\hline Cephalocereus apicicephalium E.Y. Dawson HBHs/n Chis, Oax & $\mathrm{Cl}$ & $\mathrm{Au}$ & - & 3 \\
\hline Cephalocereus totolapensis (Bravo \& T. MacDoug.) Buxb. HBHs/n & $\operatorname{Ar}$ & $\mathrm{Au}$ & - & 5 \\
\hline Disocactus macranthus (Alexander) Kimnach \& Hutchison & $\mathrm{Hi}$ & Ep & $\mathrm{Fl}$ & 5 \\
\hline
\end{tabular}


Apéndice 1. Continuación

\begin{tabular}{|c|c|c|c|c|}
\hline Familia/Especie/ Colector & FC & FN & FE & MC \\
\hline \multicolumn{5}{|l|}{ NMM800 Chis, Oax, Ver } \\
\hline Disocactus ramulosus (Salm-Dyck) Kimnach GUR \& EMS6, JAEJ247 & $\mathrm{Hi}$ & Ep & $\mathrm{Fl}$ & 2,3 \\
\hline Epiphyllum aff. crenatum MHPs/n & $\mathrm{Hi}$ & Ep & $\mathrm{E}$ & 7 \\
\hline Hylocereus minutiflorus Britton \& Rose NMM488 & $\mathrm{Hi}$ & Ep & $\mathrm{E}$ & 4 \\
\hline Hylocereus undatus (Haw.) Britton \& Rose DEB51588 & $\mathrm{Hi}$ & Ep & - & 7 \\
\hline Nopalea karwinskiana (Salm-Dyck) K. Schum. JAEJ571, 642 & $\mathrm{Hi}$ & $\mathrm{Au}, \mathrm{Ru}$ & $\mathrm{Fl}$ & 2,12 \\
\hline Opuntia deamii Rose DEB \& EMC34035 Chis, Hon, G & $\mathrm{Hi}$ & $\mathrm{Au}$ & - & 3 \\
\hline Opuntia pubescens J.C. Wendl. ex Pfeiff. DEB \& EMC34034 & $\mathrm{Hi}$ & $\mathrm{Au}$ & - & 3 \\
\hline $\begin{array}{l}\text { Peniocereus chiapensis (Bravo) Gomez-Hin. \& H.M. Hern. } \\
\quad \text { EPE2020, } 2024 \text { Chis }\end{array}$ & $\mathrm{Ab}$ & $\mathrm{Au}$ & - & 1 \\
\hline Pilosocereus leucocephalus (Poselger) Byles \& G.D. Rowley JAEJ181 & $\mathrm{Cl}$ & $\mathrm{Au}$ & $\mathrm{Fr}$ & 7 \\
\hline Rhipsalis baccifera (J.S. Muell.) Stearn JAEJ419 & $\mathrm{Hi}$ & Ep & $\mathrm{E}$ & 8 \\
\hline Selenicereus coniflorus (Weing.) Britton \& Rose JAEJ785 & $\mathrm{Cl}$ & $\mathrm{Au}$ & $\mathrm{E}$ & 4 \\
\hline $\begin{array}{l}\text { Stenocereus eichlamii (Britton \& Rose) Buxb. ex Bravo } \\
\text { HBHs/n Chis, Hon, Nic }\end{array}$ & $\operatorname{Ar}$ & $\mathrm{Au}$ & - & 1 \\
\hline Stenocereus pruinosus (Otto) Buxb. FBT4 & $\mathrm{Cl}$ & $\mathrm{Au}$ & $\mathrm{Fr}$ & 4 \\
\hline Weberocereus glaber (Eichlam) G.D. Rowley JAEJ665 & $\mathrm{Hi}$ & Ep & $\mathrm{Fl}$ & 2 \\
\hline Weberocereus glaber var. mirandae (Bravo) U. Eliasson TBMcA222 Chis & $\mathrm{Hi}$ & Ep & - & 11 \\
\hline \multicolumn{5}{|l|}{ Capparaceae } \\
\hline Capparis flexuosa (L.) L. JAEJ214, RTC, CM \& GMs/n & $\mathrm{Ab}$ & $\mathrm{Au}$ & $\mathrm{Fr}$ & 4,7 \\
\hline Capparis incana Kunth JAEJ93, RTC, CM \& GMs/n & Á & $\mathrm{Au}$ & $\mathrm{Fr}$ & 4,6 \\
\hline Capparis pringlei Briq. JAEJ219 & Á & $\mathrm{Ru}$ & $\mathrm{Fr}$ & 7 \\
\hline Capparis superba Miranda RTC, CM \& GM20088 & Á & $\mathrm{Au}$ & $\mathrm{Fr}$ & 4 \\
\hline Cleome spinosa Jacq. DEB9193, 39984, JMHJr.s/n, RTC, CM \& GMs/n, SAM34 & $\mathrm{Hi}$ & $\mathrm{Au}$ & $\mathrm{Fl}, \mathrm{Fr}$ & $2,4,5,9$ \\
\hline Cleome viscosa L. DEB26226 & $\mathrm{Hi}$ & $\mathrm{Au}$ & - & 7 \\
\hline \multicolumn{5}{|l|}{ Caprifoliaceae } \\
\hline Viburnum disjunctum C.V. Morton JAEJ129 & Á & $\mathrm{Au}$ & $\mathrm{Fl}$ & 6 \\
\hline \multicolumn{5}{|l|}{ Caricaceae } \\
\hline Jacaratia dolichaula (J.D. Smith) Woodson JLC11 & Á & $\mathrm{Au}$ & $\mathrm{Fr}$ & 4 \\
\hline Jacaratia mexicana A. DC.JAEJ568, 683 & Á & $\mathrm{Au}$ & $\mathrm{Fr}$ & 2,12 \\
\hline \multicolumn{5}{|l|}{ Caryophyllaceae } \\
\hline Arenaria lanuginosa (Michx.) Rohrb. DEB27270, 37170 & $\mathrm{Hi}$ & $\mathrm{Au}$ & - & 8,9 \\
\hline Drymaria glandulosa Bartl. DEB27271 & $\mathrm{Hi}$ & $\mathrm{Au}$ & - & 8 \\
\hline \multicolumn{5}{|l|}{ Cecropiaceae } \\
\hline Cecropia peltata L. JAEJ756 & Á & $\mathrm{Au}$ & $\mathrm{E}$ & 1 \\
\hline \multicolumn{5}{|l|}{ Celastraceae } \\
\hline $\begin{array}{l}\text { Crossopetalum parvifolium L.O. Williams ARG274, DEB27146, } \\
\text { 37226, } 39943 \text { Chis }\end{array}$ & $\mathrm{Hi}$ & $\mathrm{Au}$ & - & $2,8,9$ \\
\hline Crossopetalum tonduzii (Loes.) Lundell JAEJ748 & Á & $\mathrm{Au}$ & - & 4 \\
\hline $\begin{array}{l}\text { Crossopetalum uragoga (Jacq.) Kuntze CCC \& JIC123, DEB20108, } \\
\text { JAEJ118, JJVJ20 }\end{array}$ & $\mathrm{Ab}$ & $\mathrm{Au}$ & $\mathrm{FI}$ & $5,6,7,10$ \\
\hline $\begin{array}{l}\text { Elaeodendron trichotomum (Turcz.) Lundell DEB27175, 30028, } \\
\text { DEB, PRH \& Ers/n }\end{array}$ & Á & $\mathrm{Au}$ & - & 8,12 \\
\hline Maytenus schippii Lundell. JAEJ113 & Á & $\mathrm{Au}$ & $\mathrm{Fr}$ & 6 \\
\hline $\begin{array}{l}\text { Schaefferia frutescens Jacq. EC5970, DEB27167, 30032, DEB \& FSKs/n, } \\
\text { GD29709, GD, MSS, OT, EMS \& JDs/n, GUR, EMS \& AM26, GUR26, } \\
\text { JAEJ499, 692, JLPE, JIC \& CCC5757, PCM12 }\end{array}$ & Á & $\mathrm{Au}$ & $\mathrm{Fl}, \mathrm{Fr}$ & $\begin{array}{l}1,2,3,6 \\
8,11,12\end{array}$ \\
\hline Wimmeria bartlettii Lundell FMG7903 & Á & $\mathrm{Au}$ & - & 10 \\
\hline Wimmeria montana Lundell DEB49600, DEB \& BTKs/n Chis & Á & $\mathrm{Au}$ & - & 1 \\
\hline Wimmeria sternii Lundell CCC \& JIC124, CCC \& JLP153 & Á & $\mathrm{Au}$ & - & 7,10 \\
\hline
\end{tabular}


Apéndice 1. Continuación

\begin{tabular}{|c|c|c|c|c|}
\hline Familia/Especie/ Colector & FC & FN & FE & MC \\
\hline Zinowiewia matudae Lundell JAEJ625 & Á & $\mathrm{Au}$ & - & 2 \\
\hline \multicolumn{5}{|l|}{ Chrysobalanaceae } \\
\hline Licania platypus (Hemsl.) Fritsch. LFMJ1 & Á & $\mathrm{Au}$ & $\mathrm{Fl}$ & 10 \\
\hline \multicolumn{5}{|l|}{ Clethraceae } \\
\hline Clethra suaveolens Turcz. JAEJ681 & Á & $\mathrm{Au}$ & $\mathrm{Fl}$ & 2 \\
\hline \multicolumn{5}{|l|}{ Clusiaceae } \\
\hline Clusia flava Jacq. EPO19, JAEJ169 & Á & $\mathrm{Au}$ & $\mathrm{Fr}$ & 4,7 \\
\hline Clusia guatemalensis Hemsley MA\&MA61356, 61357 & Á & $\mathrm{Au}$ & - & 8 \\
\hline Clusia lundellii Standl. DEB28177 & Á & $\mathrm{Au}$ & - & 9 \\
\hline Clusia salvinii Donn. Sm.AM, GUR \& EMS62 & Á & $\mathrm{Au}$ & - & 2 \\
\hline Clusia mexicana Vesque FMG7753 & Á & $\mathrm{Au}$ & - & 1 \\
\hline \multicolumn{5}{|l|}{ Combretaceae } \\
\hline Bucida buceras L. JAEJ206 & Á & $\mathrm{Au}$ & $\mathrm{Fl}$ & 7 \\
\hline Bucida macrostachya Standl. LDPs/n, MAPFs/n & Á & $\mathrm{Au}$ & - & 9 \\
\hline $\begin{array}{l}\text { Combretum fruticosum (Loefl.) Stuntz CSJ; et al13245, DEB14001, } \\
\text { DEB \& ER, PRH \& DEB19767, FSKs/n, JAEJ570, JGP579, JLP } \\
\text { \&CCC6230, JLPs/n, PRH \& DEB19767 }\end{array}$ & $\mathrm{Li}$ & $\mathrm{Au}$ & $\mathrm{Fl}$ & $1,3,10,12$ \\
\hline \multicolumn{5}{|l|}{ Convolvulaceae } \\
\hline Evolvulus alsinoides (L.) L. DEB52005 & $\mathrm{Hi}$ & $\mathrm{Au}$ & - & 9 \\
\hline Evolvulus sericeus Sw. DEB41471, DEB52175 & $\mathrm{Hi}$ & $\mathrm{Au}$ & - & 11 \\
\hline Ipomoea alba L. DEB33816, 41555 & $\mathrm{Li}$ & $\mathrm{Au}$ & $\mathrm{FI}$ & 2,11 \\
\hline Ipomoea costellata Torr. DEB13875, JAEJ427 & $\mathrm{Li}$ & $\mathrm{Au}$ & $\mathrm{Fl}$ & 8,10 \\
\hline Ipomoea dimorphophylla Greenm. DDLO42 & $\mathrm{Li}$ & $\mathrm{Au}$ & - & 11 \\
\hline Ipomoea hederifolia L. DEB13856, JAEJ431, JCM13 & $\mathrm{Li}$ & $\mathrm{Au}$ & $\mathrm{Fl}$ & 8,10 \\
\hline Ipomoea heterodoxa Standl. \& Steyerm. DEB37254 & $\mathrm{Li}$ & $\mathrm{Au}$ & - & 9 \\
\hline Ipomoea indica (Burm.) Merr. JAEJ345 & $\mathrm{Li}$ & $\mathrm{Au}$ & $\mathrm{Fl}$ & 9 \\
\hline Ipomoea leucotricha Donn. Sm. AMD \&PEGs/n & $\mathrm{Li}$ & $\mathrm{Au}$ & $\mathrm{Fl}$ & 11 \\
\hline $\begin{array}{l}\text { Ipomoea murucoides Roem. \& Schult. DEB30017, DEB, } \\
\text { PRH \& Ers/n, JAEJ581 }\end{array}$ & Á & $\mathrm{Au}$ & $\mathrm{Fl}$ & 1,12 \\
\hline $\begin{array}{l}\text { Ipomoea nil (L.) Roth DEB13899, 39920, DDLO42-BIS, } \\
\text { DTR27, JAEJ373, JCM3 }\end{array}$ & $\mathrm{Li}$ & $\mathrm{Au}$ & $\mathrm{Fl}$ & $5,8,9,10,11$ \\
\hline $\begin{array}{l}\text { Ipomoea pauciflora M. Martens \& Galeotti DEB41492, DEB } \\
\text { \& FSKs/n, JAEJ566 }\end{array}$ & $\mathrm{Li}$ & $\mathrm{Au}$ & $\mathrm{Fl}$ & 11,12 \\
\hline Ipomoea pedicellaris Benth. DEB13871 & $\mathrm{Li}$ & $\mathrm{Au}$ & - & 10 \\
\hline Ipomoea purpurea (L.) Roth. JAEJ314, 340, JLOH42 & $\mathrm{Li}$ & $\mathrm{Au}$ & $\mathrm{Fl}$ & 5,9 \\
\hline Ipomoea ramosissima (Poir.) Choisy JBZ18 & $\mathrm{Li}$ & $\mathrm{Au}$ & - & 11 \\
\hline Ipomoea seducta House AMDs/n & $\mathrm{Li}$ & $\mathrm{Au}$ & $\mathrm{Fl}$ & 1 \\
\hline Ipomoea setosa Ker Gawl. DEB41477, JAEJ352 & $\mathrm{Li}$ & $\mathrm{Au}$ & $\mathrm{FI}$ & 9,11 \\
\hline Ipomoea suffulta (Kunth) G. Don DEB52191 & $\mathrm{Li}$ & $\mathrm{Au}$ & $\mathrm{Fl}$ & 8 \\
\hline Ipomoea tiliacea (Willd.) Choisy DEB41491 & $\mathrm{Li}$ & $\mathrm{Au}$ & $\mathrm{Fl}$ & 11 \\
\hline Ipomoea villifera House DEB13898, DEB \& ARS21575, DEB \& FA48465 & $\mathrm{Li}$ & $\mathrm{Au}$ & $\mathrm{Fl}$ & $10,11,12$ \\
\hline Jacquemontia nodiflora (Desr.) G. Don DEB13849 & $\mathrm{Li}$ & $\mathrm{Au}$ & $\mathrm{Fl}$ & 10 \\
\hline Jacquemontia pentantha (Jacq.) G. Don DEB13895, 37186 & $\mathrm{Li}$ & $\mathrm{Au}$ & $\mathrm{Fl}$ & 9,10 \\
\hline Jacquemontia polyantha (Schltdl. \& Cham.) Hallier f. EUVP11 & $\mathrm{Li}$ & $\mathrm{Au}$ & $\mathrm{Fl}$ & 10 \\
\hline Jacquemontia sphaerostigma (Cav.) Rusby JCM6 & $\mathrm{Li}$ & $\mathrm{Au}$ & $\mathrm{Fl}$ & 8 \\
\hline Merremia quinquefolia (L.) Hallier f. AM75, CSJ; et al13262 & $\mathrm{Li}$ & $\mathrm{Au}$ & $\mathrm{FI}$ & 2,10 \\
\hline $\begin{array}{l}\text { Merremia umbellata (L.) Hallieri f. AM65, DEB41527, DEB } \\
\quad \text { \& RFT30397, DTR28 }\end{array}$ & $\mathrm{Li}$ & $\mathrm{Au}$ & $\mathrm{Fl}$ & $2,5,11,12$ \\
\hline $\begin{array}{l}\text { Operculina pteripes (G. Don) O’Donell CRB266, CSJ; et al13300, } \\
\text { DEB41490, DEB, ER \& PH13504, EE43, JCM9 }\end{array}$ & $\mathrm{Li}$ & $\mathrm{Au}$ & $\mathrm{Fl}$ & $8,10,11$ \\
\hline
\end{tabular}


Apéndice 1. Continuación

\begin{tabular}{|c|c|c|c|c|}
\hline Familia/Especie/ Colector & FC & FN & FE & MC \\
\hline Quamoclit cholulensis (Kunth) G. Don DEB13902 & $\mathrm{Li}$ & $\mathrm{Au}$ & $\mathrm{Fl}$ & 10 \\
\hline Turbina corymbosa (L.) Raf. AM, GUR \& EMS101, CRB362, JAEJ549 & $\mathrm{Li}$ & $\mathrm{Au}$ & $\mathrm{Fl}$ & $2,11,12$ \\
\hline \multicolumn{5}{|l|}{ Crassulaceae } \\
\hline Echeveria prunina Kimn. \& Moran DEB30020 & $\mathrm{Li}$ & $\mathrm{Au}$ & - & 12 \\
\hline \multicolumn{5}{|l|}{ Cucurbitaceae } \\
\hline Cayaponia attenuata (Hook. \& Arn.) Cogn. RML905 & $\mathrm{Li}$ & $\mathrm{Au}$ & - & 5 \\
\hline Cayaponia racemosa (Mill.) Cogn. DEB \& JLS47039 & $\mathrm{Li}$ & $\mathrm{Au}$ & - & 10 \\
\hline Cyclanthera ribiflora (Schltdl.) Cogn. CCCs/n, DEB \& BB55474, JLPE \& CCC & $\mathrm{Li}$ & $\mathrm{Au}$ & - & 10,11 \\
\hline Echinopepon horridus Naudin DEB13896 & $\mathrm{Li}$ & $\mathrm{Au}$ & - & 10 \\
\hline Echinopepon milleflorus Naudin JAEJ386 & $\mathrm{Li}$ & $\mathrm{Au}$ & $\mathrm{Fl}, \mathrm{Fr}$ & 11 \\
\hline Luffa cylindrica M. Roem. DEB20194, DEB \& JLS47036 & $\mathrm{Li}$ & $\mathrm{Au}$ & - & 10 \\
\hline Melothria pendula L. AM, GUR \& EMS67, ARG947, HSG, JAEJ442 & $\mathrm{Li}$ & $\mathrm{Au}$ & $\mathrm{Fl}, \mathrm{Fr}$ & $2,9,10$ \\
\hline Polyclathra cucumerina Bertol. ARG1149 & $\mathrm{Li}$ & $\mathrm{Au}$ & - & 11 \\
\hline Rytidostylis ciliata (Cogn.) Kuntze DEB14685, DEB \& FSKs/n & $\mathrm{Li}$ & $\mathrm{Au}$ & - & 8 \\
\hline Rytidostylis gracilis Hook. \& Arn. ARG948, DEB39947, 41485, JAEJ372 & $\mathrm{Li}$ & $\mathrm{Au}$ & $\mathrm{Fl}, \mathrm{Fr}$ & $8,9,11$ \\
\hline Schizocarpum attenuatum Cogn. \& Rose DEB13872 & $\mathrm{Li}$ & $\mathrm{Au}$ & - & 10 \\
\hline \multicolumn{5}{|l|}{ Ericaceae } \\
\hline Arbutus xalapensis Kunth DEB50278 & Á & $\mathrm{Au}$ & - & 3 \\
\hline Cavendishia crassifolia (Benth.) HemsI. RML914 & $\mathrm{Ab}$ & $\mathrm{Au}$ & - & 5 \\
\hline Comarostaphylis discolor (Hook.) Diggs DEB50295 & $\mathrm{Ab}$ & $\mathrm{Au}$ & - & 3 \\
\hline \multicolumn{5}{|l|}{ Erythroxylaceae } \\
\hline Erythroxylum havanense Jacq.DEB39948, JAEJ90, JAEJ168, EL \& RFT7257 & $\mathrm{Ab}$ & $\mathrm{Au}$ & $\mathrm{Fr}$ & $6,7,9$ \\
\hline Erythroxylum rotundifolium Lunan DEB14015, 27142, 28176, JAEJ128 & Á & $\mathrm{Au}$ & $\mathrm{Fl}$ & $6,8,9,10$ \\
\hline \multicolumn{5}{|l|}{ Euphorbiaceae } \\
\hline Acalypha alopecuroides Jacq.DEB37205, JAEJ \& ALP286 & $\mathrm{Hi}$ & $\mathrm{Au}$ & $\mathrm{Fl}$ & 8,9 \\
\hline Acalypha arvensis Poepp.DEB51525 & $\mathrm{Hi}$ & $\mathrm{Au}$ & $\mathrm{Fl}$ & 7 \\
\hline $\begin{array}{l}\text { Acalypha chiapensis Brandegee DEB \& FSKs/n, DEB51516, } \\
\text { JAEJ224, 233, } 787 \text { Chis }\end{array}$ & $\mathrm{Ab}, \mathrm{Hi}$ & $\mathrm{Au}, \mathrm{Ru}$ & $\mathrm{Fr}$ & 7 \\
\hline Acalypha diversifolia Jacq.DEB51519, JAEJ \& ALP277 & $\mathrm{Hi}$ & $\mathrm{Au}$ & $\mathrm{FI}$ & 7,8 \\
\hline Acalypha langiana Müll. Arg.DEB10638, JAEJ265, JAEJ \& ALP134, SAM41 & $\mathrm{Hi}$ & $\mathrm{Au}$ & $\mathrm{Fl}$ & $5,7,8$ \\
\hline Acalypha macrostachya Jacq.AM88, DEB33793, JAEJ540 & $\mathrm{Ab}$ & $\mathrm{Au}$ & $\mathrm{Fl}$ & 2,12 \\
\hline Acalypha mollis Kunth EUVP13 & $\mathrm{Ab}$ & $\mathrm{Au}$ & - & 10 \\
\hline Acalypha setosa A. Rich. DEB52148 & $\mathrm{Hi}$ & $\mathrm{Au}$ & - & 8 \\
\hline Acalypha unibracteata Müll. Arg.JAEJ218, JAEJ783 & $\mathrm{Hi}$ & $\mathrm{Au}, \mathrm{Ru}$ & $\mathrm{Fl}$ & 1,7 \\
\hline Acalypha villosa Jacq.JG4 & $\mathrm{Hi}$ & $\mathrm{Au}$ & - & 9 \\
\hline Adelia barbinervis Schltdl. \& Cham.JAEJ366 & $\mathrm{Ab}$ & $\mathrm{Au}$ & $\mathrm{Fl}$ & 9 \\
\hline Argythamnia guatemalensis Müll. Arg.FMG7812 & $\mathrm{Ab}$ & $\mathrm{Au}$ & - & 1 \\
\hline $\begin{array}{l}\text { Chamaesyce densiflora (Klotzsch \& Garcke) Millsp.DEB, ER } \\
\quad \text { \& PH13503, GD, MSS, OT, EMS \& JDs/n }\end{array}$ & $\mathrm{Hi}$ & $\mathrm{Au}$ & - & 10 \\
\hline Chamaesyce hypericifolia (L.) Millsp. DEB10639, 41503. & $\mathrm{Hi}$ & $\mathrm{Au}$ & - & 7,11 \\
\hline Chamaesyce villifera (Scheele) Small DEB37218 & $\mathrm{Hi}$ & $\mathrm{Au}$ & - & 9 \\
\hline Cnidoscolus chayamansa McVaugh JAEJ91 & $\mathrm{Hi}$ & $\mathrm{Au}$ & $\mathrm{Fl}$ & 6 \\
\hline Cnidoscolus souzae McVaugh DTR32, RCC28 & $\mathrm{Ab}$ & $\mathrm{Au}$ & $\mathrm{Fl}$ & 5 \\
\hline Cnidoscolus tubulosus (Müll. Arg.) I.M. Johnst. DEB9024 & $\mathrm{Ab}$ & $\mathrm{Au}$ & - & 2 \\
\hline Croton aff. schiedeanus PRZ28 & Á & $\mathrm{Au}$ & - & 1 \\
\hline Croton arboreus Millsp.GUR9 & Á & $\mathrm{Au}$ & $\mathrm{Fr}$ & 2 \\
\hline Croton ciliatoglandulifer Ortega JAEJ211, JCM10 & $\mathrm{Hi}$ & $\mathrm{Au}$ & $\mathrm{Fl}$ & 7,8 \\
\hline Croton conspurcatus Schltdl.JAEJ159 & $\mathrm{Hi}$ & $\mathrm{Au}$ & - & 7 \\
\hline Croton draco Schltdl.AM, GUR \& EMS78 & Á & $\mathrm{Au}$ & $\mathrm{Fl}$ & 2 \\
\hline Croton guatemalensis Lotsy ARP114, JAEJ229 & Á & $\mathrm{Au}$ & $\mathrm{Fr}$ & 1,7 \\
\hline
\end{tabular}


Apéndice 1. Continuación

\begin{tabular}{|c|c|c|c|c|}
\hline Familia/Especie/ Colector & FC & FN & FE & MC \\
\hline Croton oerstedianus Müll. Arg.JAEJ116 & Á & $\mathrm{Au}$ & $\mathrm{Fl}$ & 6 \\
\hline Croton repens Schltdl. DEB52195 & $\mathrm{Hi}$ & $\mathrm{Au}$ & - & 8 \\
\hline Croton schiedeanus Schlecht. OFS1056 & Á & $\mathrm{Au}$ & - & 1 \\
\hline Croton xalapensis Kunth DEB52200, JAEJ227 & $\mathrm{Ab}$ & $\mathrm{Au}$ & $\mathrm{Fr}$ & 7,8 \\
\hline $\begin{array}{l}\text { Dalembertia triangularis Müll. Arg. DEB13835, 27141, 52162, 70043, } \\
\text { DEB \& ARS21572, DEB, ER \& PH13391,13502, DEB \& FSKs/n, } \\
\text { JAEJ \& ALP257 }\end{array}$ & $\mathrm{Ab}, \mathrm{Hi}$ & $\mathrm{Au}$ & $\mathrm{Fr}$ & $8,9,10,11$ \\
\hline Drypetes lateriflora (Sw.) Krug \& Urb. FMG7661 & Á & $\mathrm{Au}$ & - & 1 \\
\hline Euphorbia ariensis Kunth GUR31-bis & $\mathrm{Ab}$ & $\mathrm{Au}$ & $\mathrm{Fr}$ & 3 \\
\hline Euphorbia cyathophora Murray DEB13852, JAEJ \& ALP245 & $\mathrm{Hi}$ & $\mathrm{Au}$ & $\mathrm{Fr}$ & 10 \\
\hline $\begin{array}{l}\text { Euphorbia graminea Jacq. DEB10661, 13854, 13923, 37156, DEB \& } \\
\text { ARS21570, DEB, ER \& PH13392, 13527, EMS, OT, MSS \& GDs/n, } \\
\text { JAEJ507, MELV, ARG, JCS, RZ \& GDs/n, PRZ; et als/n, REGR55 }\end{array}$ & $\mathrm{Hi}$ & $\mathrm{Au}$ & $\mathrm{Fl}$ & $1,7,9,10,11$ \\
\hline Euphorbia heterophylla L. DEB14691, JAEJ295, JAEJ \& ALP293 & $\mathrm{Hi}$ & $\mathrm{Au}$ & $\mathrm{Fl}$ & 8 \\
\hline $\begin{array}{l}\text { Euphorbia leucocephala Lotsy CRB358, DEB13844,DEB, ER \& } \\
\text { PH13520, DEB \& FSKs/n, DEB, PRH \& Ers/n, EMS, OT, MSS, GD } \\
\text { \& JD, FDAL22, JAEJ565 }\end{array}$ & $\mathrm{Ab}, \mathrm{Hi}$ & $\mathrm{Au}$ & $\mathrm{Fl}$ & $10,11,12$ \\
\hline $\begin{array}{l}\text { Euphorbia macropus (Klotzsch \& Garcke) Boiss. DEB13870, DEB, } \\
\qquad \text { ER \& PH13496, DEB, PRH \& Ers/n }\end{array}$ & $\mathrm{Hi}$ & $\mathrm{Au}$ & - & 10 \\
\hline Euphorbia ocymoidea L. DEB, ER \& PH13513 & $\mathrm{Hi}$ & $\mathrm{Au}$ & - & 10 \\
\hline Euphorbia pseudofulva Miranda DEB39950 Chis & Á & $\mathrm{Au}$ & - & 9 \\
\hline Euphorbia pulcherrima Willd. ex Klotzsch ACH14, DEB41478, JRV6 & $\mathrm{Ab}$ & $\mathrm{Au}$ & $\mathrm{Fl}$ & 11,12 \\
\hline Euphorbia schlechtendalii Boiss. DEB14009, DEB \& FSKs/n, JAEJ715 & $\mathrm{Ab}$ & $\mathrm{Au}$ & $\mathrm{Fr}$ & 3,10 \\
\hline Euphorbia thymifolia L. AM, GUR \& EMS74 & $\mathrm{Hi}$ & $\mathrm{Au}$ & - & 2 \\
\hline Gymnanthes actinostemoides Müll. Arg.GUR9, 36, 38, JAEJ105, 412, 447 & Á & $\mathrm{Au}$ & $\mathrm{Fr}$ & $2,6,8,10$ \\
\hline Manihot aesculifolia (Kunth) Pohl JAEJ188, 256 & $\mathrm{Hi}$ & $\mathrm{Au}$ & $\mathrm{Fl}$ & 7,8 \\
\hline Manihot esculenta Crantz DEB51584 & $\mathrm{Ab}$ & $\mathrm{Au}$ & - & 7 \\
\hline Manihot rhomboidea Müll. Arg. DEB \& FSKs/n, LH, MM \& JJs/n & $\mathrm{Ab}$ & $\mathrm{Au}$ & - & 6,8 \\
\hline $\begin{array}{l}\text { Manihot rhomboidea subsp. microcarpa (Müll. Arg.) D.J. Rogers } \\
\quad \text { \& Appan AGP2589, DEB10633, 27262, 39931, 70253, OFC236 }\end{array}$ & $\mathrm{Ab}$ & $\mathrm{Au}$ & - & $7,8,9$ \\
\hline $\begin{array}{l}\text { Pedilanthus calcaratus Schltdl. DEB14012, 49601, 9023, GUR, EMS } \\
\quad \text { \& AM37, JAEJ635, } 743\end{array}$ & $\mathrm{Ab}$ & $\mathrm{Au}$ & $\mathrm{Fl}$ & $1,2,4,10$ \\
\hline Phyllanthus grandiflorus Spreng. DEB52187, DEB \& ARS21563, JAEJ324 & $\mathrm{Ab}$ & $\mathrm{Au}$ & $\mathrm{Fr}$ & $8,9,11$ \\
\hline Phyllanthus tequilensis B.L. Rob. \& Greenm. AGP2579 & $\mathrm{Ab}$ & $\mathrm{Au}$ & - & 8 \\
\hline Sapium schippii Croizat FMG7754 & Á & $\mathrm{Au}$ & - & 1 \\
\hline Sebastiania cruenta (Standl. \& Steyerm.) Miranda DEB70060, GD. Et al29720 & $\mathrm{Ab}$ & $\mathrm{Au}$ & - & 9,11 \\
\hline Stillingia acutifolia (Benth.) Benth. ex Hemsl. JMHJrs/n & $\mathrm{Ab}$ & $\mathrm{Au}$ & - & 9 \\
\hline Tragia nepetifolia Cav. DEB39968 & $\mathrm{Hi}$ & $\mathrm{Au}$ & - & 9 \\
\hline \multicolumn{5}{|l|}{ Fabaceae } \\
\hline Acacia angustissima (Mill.) Kuntze VMSL1106, JAEJ \& JMM269,ARG935 & Á, $\mathrm{Ab}$ & $\mathrm{Au}$ & $\mathrm{Fl}$ & $1,8,9$ \\
\hline Acacia cochliacantha Humb. \& Bonpl. ex Willd.AM, GUR \& EMS17, RCC24 & Á & $\mathrm{Au}$ & $\mathrm{Fl}, \mathrm{Fr}$ & 2,5 \\
\hline Acacia collinsii Saff. MSS, EMS \& ARG13153, MGRG112 & Á & $\mathrm{Au}$ & - & 2,4 \\
\hline Acacia cornigera (L.) Willd. ARG516, JLOH48 & $\mathrm{Ab}$ & $\mathrm{Au}$ & $\mathrm{Fl}, \mathrm{Fr}$ & 4,5 \\
\hline Acacia costaricensis Schenck JAEJ153 & Á & $\mathrm{Au}$ & $\mathrm{Fl}$ & 7 \\
\hline Acacia farnesiana (L.) Willd.AM, GUR \& EMS64, JAEJ86 & Á & $\mathrm{Au}$ & $\mathrm{Fr}$ & 2,6 \\
\hline $\begin{array}{l}\text { Acacia pennatula (Schltdl. \& Cham.) Benth. DTR29, JAEJ548, } \\
\text { JLOH34, PTL5884 }\end{array}$ & $\mathrm{Ab}$ & $\mathrm{Au}$ & $\mathrm{Fl}, \mathrm{Fr}$ & $3,5,12$ \\
\hline Acacia pringlei Rose EMS20067 & Á & $\mathrm{Au}$ & $\mathrm{Fl}, \mathrm{Fr}$ & 2 \\
\hline Acaciella villosa (Sw.) Britton \& Rose EE30 & $\mathrm{Ab}$ & $\mathrm{Au}$ & $\mathrm{Fl}$ & 8 \\
\hline Aeschynomene americana var. americana L. DEB13919, 41468 & $\mathrm{Hi}$ & $\mathrm{Au}$ & - & 10,11 \\
\hline
\end{tabular}


Apéndice 1. Continuación

\begin{tabular}{|c|c|c|c|c|}
\hline Familia/Especie/ Colector & FC & FN & FE & MC \\
\hline Aeschynomene compacta Rose DEB39932, 41515 & $\mathrm{Ab}$ & $\mathrm{Au}$ & - & 9,11 \\
\hline Albizia adinocephala (Donn. Sm.) Britton \& Rose ex Record ERB27 & Á & $\mathrm{Au}$ & - & 10 \\
\hline Albizia tomentosa (Micheli) Standl. ARG521 & Á & $\mathrm{Au}$ & $\mathrm{Fl}, \mathrm{Fr}$ & 5 \\
\hline Ateleia chicoasensis J. Linares JLL, HI \& PTL4333 Dep & Á & $\mathrm{Au}$ & $\mathrm{Fr}$ & 2 \\
\hline Bauhinia cookii Rose JAEJ496 & Á & $\mathrm{Au}$ & $\mathrm{E}$ & 11 \\
\hline $\begin{array}{l}\text { Bauhinia divaricata L. CHP3045, DEB13839, 39955, 9021, EC5956, } \\
\text { EMS20078, JAEJ547, MRV16 }\end{array}$ & $\mathrm{Ab}$ & $\mathrm{Au}$ & $\mathrm{Fl}, \mathrm{Fr}$ & $\begin{array}{l}1,2,3,9 \\
10,11,12\end{array}$ \\
\hline Caesalpinia pulcherrima (L.) Sw. AM, GUR \& EMS16, JAEJ68, MDRS1 & Á & $\mathrm{Au}$ & $\mathrm{Fl}, \mathrm{Fr}$ & $2,6,8$ \\
\hline Calliandra calothyrsus Meisn. JAEJ117, JAEJ \&ALP294 & $\mathrm{Ab}$ & $\mathrm{Au}$ & $\mathrm{Fl}$ & 6,8 \\
\hline Calliandra formosa (Kunth) Benth. DEB10635 & $\mathrm{Ab}$ & $\mathrm{Au}$ & - & 7 \\
\hline Calliandra grandiflora (L' Hér.) Benth. RCC25 & $\mathrm{Ab}$ & $\mathrm{Au}$ & $\mathrm{Fl}$ & 5 \\
\hline Calliandra hirsuta (G. Don) Benth. SD2465 & & $\mathrm{Au}$ & - & 1 \\
\hline $\begin{array}{l}\text { Calliandra houstoniana (Mill.) Standl.ACH19, ARG892, } \\
\text { DDLO41, DN5482, RORRs/n }\end{array}$ & $\mathrm{Ab}$ & $\mathrm{Au}$ & $\mathrm{Fl}$ & $1,3,5,9,11$ \\
\hline Canavalia glabra (M. Martens \& Galeotti) J.D. Sauer DEB \& FA48457 & $\mathrm{Li}$ & $\mathrm{Au}$ & - & 12 \\
\hline $\begin{array}{l}\text { Canavalia villosa Benth. DEB11610, EMS20157, JAEJ503, MSS, JD, } \\
\text { OT \& EMSs/n, MSS, TPR \& PB11403 }\end{array}$ & $\mathrm{Li}$ & $\mathrm{Au}$ & $\mathrm{Fl}$ & $4,10,11,12$ \\
\hline Centrosema galeotii Fantz DEB41511, 70055 & $\mathrm{Li}$ & $\mathrm{Au}$ & - & 9,11 \\
\hline Centrosema plumieri (Turpin ex Pers.) Benth. DEB33809 & $\mathrm{Li}$ & $\mathrm{Au}$ & - & 2 \\
\hline Centrosema pubescens Benth. DEB 41511, MSS11622 & $\mathrm{Li}$ & $\mathrm{Au}$ & - & - \\
\hline $\begin{array}{l}\text { Centrosema schottii (Millsp.) K. Schum. DEB13838, 41514, DEB } \\
\text { \& JLS47037, DEB, ER \& PH13519, MSS 11406, MSS, EMS } \\
\text { \& ARG13146, MSS, EMS, ARG \& CL11617, SAM38 }\end{array}$ & $\mathrm{Li}$ & $\mathrm{Au}$ & - & $\begin{array}{c}2,5,10 \\
11,12\end{array}$ \\
\hline Centrosema virginianum (L.) Benth. JAEJ342, 444 & $\mathrm{Li}$ & $\mathrm{Au}$ & $\mathrm{Fl}$ & 10 \\
\hline Chamaecrista desvauxii DEB39976 & $\mathrm{Hi}$ & $\mathrm{Au}$ & - & 9 \\
\hline Chamaecrista nictitans (L.) Moench CSJ, DS, RH \& ARG13310, ERB18 & $\mathrm{Ab}, \mathrm{Hi}$ & $\mathrm{Au}$ & - & 10 \\
\hline Clitoria macrophylla Wall. EUVP22 & $\mathrm{Li}$ & $\mathrm{Au}$ & $\mathrm{Fl}$ & 10 \\
\hline Cologania glabrior Rose JAEJ468 & $\mathrm{Li}$ & $\mathrm{Au}$ & $\mathrm{Fl}$ & 10 \\
\hline Crotalaria cajanifolia Kunth JAEJ370 & $\mathrm{Hi}$ & $\mathrm{Au}$ & $\mathrm{Fl}$ & 8 \\
\hline Crotalaria filifolia DEB, ER \& PH13375 & $\mathrm{Hi}$ & $\mathrm{Au}$ & $\mathrm{Fl}$ & 10 \\
\hline Crotalaria pumila DEB13842, 13843, 39979 & $\mathrm{Hi}$ & $\mathrm{Au}$ & - & 10 \\
\hline Crotalaria sagittalis L. DEB13873, JAEJ433 & $\mathrm{Hi}$ & $\mathrm{Au}$ & $\mathrm{Fr}$ & 8,10 \\
\hline Crotalaria vitellina Ker Gawl. DEB13878 & $\mathrm{Hi}$ & $\mathrm{Au}$ & - & 10 \\
\hline Cynometra retusa Britton \& Rose ARG528 & Á & $\mathrm{Au}$ & - & 5 \\
\hline Dalbergia calycina Benth. DEB9571, JAEJ582 & Á & $\mathrm{Au}$ & $\mathrm{FI}$ & 1,4 \\
\hline Dalbergia glabra (Mill.) Standl.JAEJ767 & $\mathrm{Li}$ & $\mathrm{Au}$ & $\mathrm{FI}$ & 4 \\
\hline Dalea annua Kuntze JAEJ440 & $\mathrm{Hi}$ & $\mathrm{Au}$ & $\mathrm{Fl}$ & 10 \\
\hline Dalea carthagenensis (Jacq.) J.F. Macbr. MSS, JD, OT \& EMS & $\mathrm{Hi}$ & $\mathrm{Au}$ & - & 11 \\
\hline Dalea leporina (Aiton) Hemsl. JCM5 & $\mathrm{Hi}$ & $\mathrm{Au}$ & - & 8 \\
\hline Dalea spiciformis (Rose) Bullock DEB, ER \& PH13376 & $\mathrm{Hi}$ & $\mathrm{Au}$ & - & 10 \\
\hline Desmanthus virgatus (I.) Willd. AM108 & $\mathrm{Ab}$ & $\mathrm{Au}$ & - & 2 \\
\hline Desmodium infractum DC. JAEJ365 & $\mathrm{Li}$ & $\mathrm{Au}$ & $\mathrm{Fl}$ & 9 \\
\hline Desmodium intortum (Mill.) Urb. DEB \& FSs/n & $\mathrm{Hi}$ & $\mathrm{Au}$ & - & 10 \\
\hline Desmodium nicaraguense Oerst. DEB13841, DEB \& BB55486, DEB \& FSKs/n & $\mathrm{Ab}$ & $\mathrm{Au}$ & $\mathrm{FI}$ & 10,11 \\
\hline $\begin{array}{l}\text { Desmodium plicatum Schltdl. \& Cham. DEB33770, 41529, } \\
\text { MSS, EMS \& ARG13145 }\end{array}$ & $\mathrm{Ab}$ & $\mathrm{Au}$ & $\mathrm{Fl}$ & 2,11 \\
\hline Desmodium prehensile Schltdl. JAEJ450 & $\mathrm{Hi}$ & $\mathrm{Au}$ & $\mathrm{Fl}$ & 10 \\
\hline Desmodium pringlei S. Watson DEB10645, 27265 & $\mathrm{Ab}$ & $\mathrm{Au}$ & - & 7,8 \\
\hline $\begin{array}{l}\text { Desmodium skinneri Benth. ex Hemsl. AM, GUR \& EMS105, } \\
\text { 105A, 105-bis, MAMAs/n }\end{array}$ & $\mathrm{Ab}$ & $\mathrm{Au}$ & $\mathrm{FI}$ & 2,3 \\
\hline
\end{tabular}


Apéndice 1. Continuación

\begin{tabular}{|c|c|c|c|c|}
\hline Familia/Especie/ Colector & FC & FN & FE & MC \\
\hline Desmodium tortuosum (Sw.) DC. DEB41484, JAEJ355, MSS, JD, OT, EMS & $\mathrm{Hi}$ & $\mathrm{Au}$ & $\mathrm{Fl}$ & 10,11 \\
\hline Diphysa floribunda Peyr. DEB33739, 9029 & Á & $\mathrm{Au}$ & $\mathrm{Fl}$ & 2 \\
\hline Diphysa racemosa Rose AM, GUR \& EMS20, DEB \& BTK49597 & Á & $\mathrm{Au}$ & $\mathrm{Fl}, \mathrm{Fr}$ & 1,2 \\
\hline Diphysa spinosa Rydb. JAEJ \& ALP194, JAEJ131 & $\mathrm{Ab}$ & $\mathrm{Au}$ & $\mathrm{Fl}$ & 6,7 \\
\hline Enterolobium cyclocarpum (Jacq.) Griseb. JAEJ72 & Á & $\mathrm{Au}$ & $\mathrm{Fr}$ & 6 \\
\hline Erythrina americana Mill. ARG538 & Á & $\mathrm{Au}$ & $\mathrm{Fr}$ & 5 \\
\hline Erythrina chiapasana Krukoff DEB9612 & Á & $\mathrm{Au}$ & - & 4 \\
\hline Erythrina folkersii Krukoff \& Moldenke JAEJ75, MDRS4 & Á & $\mathrm{Au}$ & $\mathrm{Fr}$ & 6,8 \\
\hline $\begin{array}{l}\text { Erythrina goldmani Standl. DEB39981, DEB, ER \& PH13511, } \\
\text { JAEJ577 Chis, G, Oax }\end{array}$ & Á & $\mathrm{Au}$ & $\mathrm{Fr}$ & $1,9,10$ \\
\hline Eysenhardtia adenostylis Baill. DEB13853 Chis, C & Á & $\mathrm{Au}$ & $\mathrm{Fl}$ & 10 \\
\hline Gliricidia ehrenbergii (Schltdl.) Rydb. DEB39954 & Á & - & - & 9 \\
\hline $\begin{array}{l}\text { Gliricidia sepium (Jacq.) Kunth ex Walp AM, GUR \& EMS2, EMS, } \\
\text { ARG, GR \& GUR20102 }\end{array}$ & Á & $\mathrm{Au}$ & $\mathrm{Fl}, \mathrm{Fr}$ & 2,4 \\
\hline Haematoxylum brasiletto H. Karst. GUR, EMS \& AM10, JAEJ643, 644 & Á, $A b$ & $\mathrm{Au}$ & $\mathrm{Fl}, \mathrm{Fr}$ & 2 \\
\hline $\begin{array}{l}\text { Hybosema robustum M. Sousa \& Lavin CEH1186, 1765, DEB39954, } \\
\text { EMS \& ARG22047, EMS, ARG \& CL13152, EMS \& MASA24178, } \\
\text { MSSNRM \& MJDG475 Dep }\end{array}$ & Á & $\mathrm{Au}$ & $\mathrm{Fl}, \mathrm{Fr}$ & $\begin{array}{c}2,4,5 \\
9,12\end{array}$ \\
\hline Indigofera mucronata Lam. DEB, ER \& PH13495, EE29,JAEJ267 & $\mathrm{Hi}$ & $\mathrm{Au}$ & $\mathrm{Fl}$ & 8,10 \\
\hline Indigofera suffruticosa Mill. DEB41516 & $\mathrm{Ab}$ & $\mathrm{Au}$ & - & 11 \\
\hline Inga chiapensis Miranda ex M. Sousa FMG7662, OT6690, 7578 Chis, Ver & - & - & - & - \\
\hline Inga micheliana Harms JAEJ494 & Á & $\mathrm{Au}$ & $\mathrm{E}$ & 11 \\
\hline Inga sapindoides Willd JAEJ375 & Á & $\mathrm{Au}$ & $\mathrm{E}$ & 8 \\
\hline $\begin{array}{l}\text { Leucaena collinsii Britton \& Rose ARG888, CSJ, DS , RH } \\
\quad \text { \& ARG13265, DEB13889, } 13890\end{array}$ & Á & $\mathrm{Au}$ & $\mathrm{Fl}$ & 9,10 \\
\hline Leucaena esculenta (Moc. \& Sessé ex DC.) Benth. JBZ19 & Á & $\mathrm{Au}$ & - & 11 \\
\hline Leucaena glauca Benth. ARG888 & Á & $\mathrm{Au}$ & $\mathrm{Fl}$ & 9 \\
\hline $\begin{array}{l}\text { Lonchocarpus acuminatus Benth. (Schltdl.) M. Sousa DEB10664, } \\
\text { GUR, EMS \& AMG19 }\end{array}$ & Á & $\mathrm{Au}$ & $\mathrm{Fr}$ & $1,2,7$ \\
\hline $\begin{array}{l}\text { Lonchocarpus guatemalensis Benth. EMS, ARG, AM \& GUR 20114, } \\
\text { 20116, 20118, MSS, EMS \& ARG13142 }\end{array}$ & Á & $\mathrm{Au}$ & $\mathrm{Fl}, \mathrm{Fr}$ & 2,4 \\
\hline Lonchocarpus lanceolatus Benth. JAEJ127 & Á & $\mathrm{Au}$ & $\mathrm{Fl}$ & 4,6 \\
\hline Lonchocarpus martinezii M. Sousa JAEJ744, PTL19758 Dep & Á & $\mathrm{Au}$ & - & - \\
\hline Lonchocarpus michelianus Pittier DEB \& FSKs/n & Á & $\mathrm{Au}$ & - & 7 \\
\hline Lonchocarpus minimiflorus Donn. Sm. CSJ, DS, RH \& ARG13301, JAEJ126 & Á & $\mathrm{Au}$ & $\mathrm{Fl}$ & 6,10 \\
\hline Lonchocarpus parviflorus Benth. PAF \& EJLTT3280 & Á & $\mathrm{Au}$ & - & 11 \\
\hline Lonchocarpus rugosus Benth. RGOs/n, ARG, RH \& PJSs/n, JAEJ313 & Á & $\mathrm{Au}$ & $\mathrm{Fr}$ & 1 \\
\hline Lonchocarpus rugosus subsp. apricus (Lundell) M. Sousa JAEJ313 & Á & $\mathrm{Au}$ & $\mathrm{Fr}$ & 9 \\
\hline Lysiloma acapulcense (Kunth) Benth. MSS, EMS \& ARG13156 & Á & $\mathrm{Au}$ & $\mathrm{Fl}$ & 2 \\
\hline Lysiloma auritum (Schltdl.) Benth. CMGH40, DN5520 & Á & $\mathrm{Au}$ & $\mathrm{Fl}$ & 5 \\
\hline $\begin{array}{l}\text { Lysiloma divaricatum (Jacq.) J.F. Macbr. ARG945,JAEJ559, JAEJ } \\
\qquad \& \text { ALP142, } 190\end{array}$ & Á, $A b$ & $\mathrm{Au}$ & Fr & $7,9,12$ \\
\hline Machaerium biovulatum Micheli DEB13908, MSS, EMS \& ARG13147 & Á & $\mathrm{Au}$ & $\mathrm{Fl}$ & 2,10 \\
\hline Machaerium chiapense Brandegee DEB \& JLS47041, JAEJ95 & Á & $\mathrm{Au}, \mathrm{Ru}$ & $\mathrm{Fl}$ & 6,10 \\
\hline Macroptilium gibbosifolium (Ortega) A. Delgado JMHJr11 & $\mathrm{Li}$ & $\mathrm{Au}$ & $\mathrm{Fl}$ & 6 \\
\hline Marina scopa Barneby MSS, OT, GD, JD \& EMS12826, 12830 & $\mathrm{Hi}$ & $\mathrm{Au}$ & - & 11 \\
\hline Mimosa albida Humb. \& Bonpl. ex Willd. AM69, HMC6, JAEJ448 & $\mathrm{Ab}$ & $\mathrm{Au}$ & $\mathrm{Fl}$ & $2,10,11$ \\
\hline $\begin{array}{l}\text { Mimosa hondurana Britton AM, GUR \& EMS85, JAEJ435, MSS, EMS } \\
\quad \text { \& ARG13149, MSS, TPR \& PB11609 Chis, C }\end{array}$ & $\mathrm{Ab}, \mathrm{Li}$ & $\mathrm{Au}$ & $\mathrm{Fl}$ & $2,10,12$ \\
\hline Mimosa microphylla Dryand. ex Sm. LFMJ17 & $\mathrm{Hi}$ & $\mathrm{Au}$ & $\mathrm{Fl}$ & 10 \\
\hline
\end{tabular}


Apéndice 1. Continuación

\begin{tabular}{|c|c|c|c|c|}
\hline Familia/Especie/ Colector & FC & FN & FE & MC \\
\hline Mimosa pigra L. JAEJ82 & $\mathrm{Ab}$ & $\mathrm{Au}$ & $\mathrm{Fr}$ & 6 \\
\hline Mimosa psilocarpa B.L. Rob. DEB27184 Chis, Oax & $\mathrm{Ab}$ & $\mathrm{Au}$ & $\mathrm{Fl}$ & 8 \\
\hline Minkelersia galactioides M. Martens \& Galeotti GUR, EMS \& AM92 & $\mathrm{Li}$ & $\mathrm{Au}$ & - & 2 \\
\hline Mucuna argyrophylla Standl. DEB51521 & $\mathrm{Li}$ & $\mathrm{Au}$ & $\mathrm{Fl}$ & 7 \\
\hline Nissolia fruticosa Jacq. ARG, RH \& LH1729, DEB \& FSKs/n & $\mathrm{Li}$ & $\mathrm{Au}$ & $\mathrm{FI}$ & 1,10 \\
\hline $\begin{array}{l}\text { Nissolia fruticosa var. fruticosa Jacq. ARG; et al.1729, DEB10666, } \\
\text { 13861, 39958, JAEJ305 }\end{array}$ & $\mathrm{Li}$ & $\mathrm{Au}$ & $\mathrm{Fl}$ & $7,8,9,10$ \\
\hline Pachyrhizus erosus (L.) Urb. ARG; et al.927, CEMM16, LFMJ7 & Á, $\mathrm{Hi}, \mathrm{Li}$ & $\mathrm{Au}$ & $\mathrm{Fl}, \mathrm{Fr}$ & 9,10 \\
\hline Phaseolus lunatus L. MSS, TPR, PB \& CL11611 & $\mathrm{Li}$ & $\mathrm{Au}$ & $\mathrm{Fl}$ & 12 \\
\hline Phaseolus microcarpus Mart. ARG \& et al.1285 & $\mathrm{Li}$ & $\mathrm{Au}$ & $\mathrm{Fl}$ & 10 \\
\hline Phaseolus vulgaris L. JAEJ472 & $\mathrm{Li}$ & $\mathrm{Au}$ & $\mathrm{Fl}$ & 9 \\
\hline Piscidia carthagenensis Jacq. MSS, EMS \& ARG13151,ARG, MSS \& EMS278 & Á & $\mathrm{Au}$ & $\mathrm{Fl}, \mathrm{Fr}$ & 2 \\
\hline Piscidia piscipula (L.) Sarg. DEB \& FSKs/n & Á & $\mathrm{Au}$ & - & 4 \\
\hline Pithecellobium albicaule Britton \& Rose MSS, EMS \& ARG13144 & Á & $\mathrm{Au}$ & $\mathrm{Fl}$ & 2 \\
\hline Pithecellobium dulce (Roxb.) Benth AM, GUR \& EMS32, JAEJ70, SAM21 & Á & $\mathrm{Au}$ & $\mathrm{Fr}$ & $2,5,6$ \\
\hline $\begin{array}{l}\text { Pithecellobium lanceolatum (Humb. \& Bonpl. ex Willd.) Benth. } \\
\text { DEB51951, EMS, ARG, AM \& GUR20119 }\end{array}$ & Á & $\mathrm{Au}$ & $\mathrm{Fr}$ & 4,8 \\
\hline Pterocarpus rohrii Vahl AM, GUR \& EMS49 & Á & $\mathrm{Au}$ & - & 2 \\
\hline Ramirezella strobilophora (B.L. Rob.) Rose JAEJ261 & $\mathrm{Li}$ & $\mathrm{Au}$ & $\mathrm{Fl}$ & 8 \\
\hline Rhynchosia edulis Griseb. MSS, EMS, ARG \& CL11621 & $\mathrm{Li}$ & $\mathrm{Au}$ & - & 12 \\
\hline Rhynchosia latifolia Nutt. ex Torr. \& A. Gray EMS, ARG, AM \& GUR20123 & $\mathrm{Li}$ & $\mathrm{Au}$ & $\mathrm{Fr}$ & 4 \\
\hline $\begin{array}{l}\text { Rhynchosia longeracemosa M. Martens \& Galeotti AM, GUR \& EMS80, } \\
\text { 80-bis, AM, GUR \& EMS92, DEB33788, MSS, JJD, OT Y\& EMSs/n }\end{array}$ & $\mathrm{Li}$ & $\mathrm{Au}$ & $\mathrm{FI}$ & 2,11 \\
\hline $\begin{array}{l}\text { Rhynchosia minima (L.) DC. DEB39970, 39971, DEB, ER \& PH13535, } \\
\text { DEB, PRH \& Ers/n, MSS, EMS \& ARG13148, MSS, TPR, PB } \\
\text { \& CL11614, LFMJ5 }\end{array}$ & $\mathrm{Li}$ & $\mathrm{Au}$ & $\mathrm{Fl}, \mathrm{Fr}$ & $2,9,10,12$ \\
\hline $\begin{array}{l}\text { Senna atomaria (L.) H.S. Irwin \& Barneby AM, GUR \& EMS7, AM, GUR } \\
\text { \& EMS27, EMS20106, ERB26, JAEJ560, JAEJ \& JMM69, LFMJ18, MDRS2 }\end{array}$ & Á, Ab & $\mathrm{Au}$ & $\mathrm{Fl}, \mathrm{Fr}$ & $\begin{array}{c}2,4,7,8 \\
10,12\end{array}$ \\
\hline Senna holwayana (Rose) H.S. Irwin \& Barneby AM41 & Á & $\mathrm{Au}$ & $\mathrm{FI}$ & 2 \\
\hline Senna incarnata (Pav. ex Benth.) H.S. Irwin \& Barneby MSS, TPR \& PB11607 & $\mathrm{Ab}$ & $\mathrm{Au}$ & $\mathrm{Fl}$ & 12 \\
\hline $\begin{array}{l}\text { Senna nicaraguensis (Benth.) H.S. Irwin \& Barneby AM, GUR \& EMS15, } \\
\text { CSJ DS, RH \& ARG13263, DEB, ER \& PH13515, JAEJ564 }\end{array}$ & Á & $\mathrm{Au}$ & $\mathrm{Fl}, \mathrm{Fr}$ & $2,10,12$ \\
\hline Senna occidentalis (L.) Link ERB27, JAEJ407, 467 & Á, $A b$ & $\mathrm{Au}$ & $\mathrm{Fl}$ & 8,10 \\
\hline Senna pallida (Vahl) H.S. Irwin \& Barneby AM, GUR \& EMS72, LFMJ16 & $\mathrm{Ab}$ & $\mathrm{Au}$ & $\mathrm{Fl}, \mathrm{Fr}$ & 2,10 \\
\hline Senna pallida var. pallida DEB28174 & $\mathrm{Ab}$ & $\mathrm{Au}$ & - & 9 \\
\hline Senna pentagonia var. pentagonia DEB13848 & $\mathrm{Ab}$ & $\mathrm{Au}$ & $\mathrm{Fl}$ & 10 \\
\hline Senna reticulata (Willd.) H.S. Irwin \& Barneby EMS, ARG, GR \& GUR20094 & $\mathrm{Ab}$ & $\mathrm{Au}$ & $\mathrm{Fr}$ & 4 \\
\hline $\begin{array}{l}\text { Senna skinneri (Benth.) H.S Irwin \& Barneby DEB, ER \& PH13534, } \\
\text { JAEJ254,VAGL22 }\end{array}$ & Á & $\mathrm{Au}$ & $\mathrm{FI}$ & $8,10,11$ \\
\hline Senna tonduzii (Standl.) H.S. Irwin \& Barneby CSJ DS, RH \& ARG13306 & Á & $\mathrm{Au}$ & $\mathrm{FI}$ & 10 \\
\hline $\begin{array}{l}\text { Senna uniflora (Mill.) H.S. Irwin \& Barneby DEB, ER \& PH13514, } \\
\text { JAEJ354, OFC } 231\end{array}$ & $\mathrm{Hi}$ & $\mathrm{Au}$ & $\mathrm{FI}$ & $8,9,10$ \\
\hline Stizolobium pruriens var. pruriens DEB41487, 41528 & $\mathrm{Li}$ & $\mathrm{Au}$ & $\mathrm{Fl}$ & 11 \\
\hline Stylosanthes humilis Kunth DEB, ER \& PH13377 & $\mathrm{Hi}$ & $\mathrm{Au}$ & $\mathrm{Fl}$ & 10 \\
\hline Tephrosia cinerea (L.) Pers. EMS, et al.s/n, MSS11616 & $\mathrm{Hi}$ & $\mathrm{Au}$ & $\mathrm{Fl}, \mathrm{Fr}$ & 4,12 \\
\hline Tephrosia sinapou (Buc'hoz) A. Chev. DEB39980 & $\mathrm{Ab}$ & $\mathrm{Au}$ & $\mathrm{Fl}$ & 9 \\
\hline Teramnus uncinatus (L.) Sw. MSS12825 & $\mathrm{Li}$ & $\mathrm{Au}$ & - & 11 \\
\hline $\begin{array}{l}\text { Vigna adenantha (G. Mey.) Maréchal, Mascherpa \& Stainier DEB41539, } \\
\text { DEB \& JLS47045 }\end{array}$ & $\mathrm{Li}$ & $\mathrm{Au}$ & $\mathrm{FI}$ & 10,11 \\
\hline Vigna speciosa (Kunth) Verdc. DEB13913, 41542 & $\mathrm{Li}$ & $\mathrm{Au}$ & $\mathrm{Fl}$ & 10,11 \\
\hline
\end{tabular}


Apéndice 1. Continuación

\begin{tabular}{|c|c|c|c|c|}
\hline Familia/Especie/ Colector & FC & FN & FE & MC \\
\hline Zapoteca formosa subsp. formosa DEB10635 & - & $\mathrm{Au}$ & - & 7 \\
\hline Zapoteca portoricensis var. portoricensis RML915 & $\mathrm{Ab}$ & $\mathrm{Au}$ & $\mathrm{Fr}$ & 5 \\
\hline \multicolumn{5}{|l|}{ Fagaceae } \\
\hline Quercus acutifolia Née JAEJ528 & Á & $\mathrm{Au}$ & $\mathrm{E}$ & 10 \\
\hline Quercus benthamii A. DC. JAEJ437 Chis, G, Oax & Á & $\mathrm{Au}$ & $\mathrm{E}$ & 10 \\
\hline Quercus castanea Née DEB33751, 35066 & Á & $\mathrm{Au}$ & - & 2,5 \\
\hline Quercus crassifolia Humb. \& Bonpl. LMGVs/n & Á & $\mathrm{Au}$ & - & 7 \\
\hline Quercus elliptica Née.JAEJ479, 485 & Á & $\mathrm{Au}$ & $\mathrm{Fr}$ & 10 \\
\hline Quercus magnoliifolia Née JAEJ741 & Á & $\mathrm{Au}$ & $\mathrm{Fl}$ & 4 \\
\hline Quercus opaca Trel. DEB \& ARSs/n & Á & $\mathrm{Au}$ & - & 11 \\
\hline Quercus peduncularis Née DEB33787, 35063, 51682, 51689, 70022 & Á & $\mathrm{Au}$ & - & $2,5,7,9$ \\
\hline Quercus polymorpha Schltdl. \& Cham. DEB33764 & Á & $\mathrm{Au}$ & - & 2 \\
\hline Quercus purulhana Trel. DEB70256 Chis, C & Á & $\mathrm{Au}$ & - & 9 \\
\hline Quercus rugosa Née JAEJ438 & $\mathrm{Ab}$ & $\mathrm{Au}$ & $\mathrm{E}$ & 10 \\
\hline $\begin{array}{l}\text { Quercus sebifera Trel. DEB70255, DEB \& ARS21548, DEB \& FA48454, } \\
\text { JAEJ331, OT \& JLVR6685 }\end{array}$ & Á, $\mathrm{Ab}$ & $\mathrm{Au}$ & $\mathrm{Fl}, \mathrm{Fr}$ & $4,9,11,12$ \\
\hline $\begin{array}{l}\text { Quercus segoviensis Liebm. DEB27185, DEB \& ARS21554, 235163, } \\
\text { ER, PRH \& DEB19764 }\end{array}$ & Á & $\mathrm{Au}$ & $\mathrm{Fl}, \mathrm{Fr}$ & $1,8,11$ \\
\hline \multicolumn{5}{|l|}{ Flacourtiaceae } \\
\hline Casearia corymbosa Kunth.DEB26218, 39965, Els/n, JAEJ73, 88 & $\mathrm{Ab}$ & $\mathrm{Au}$ & $\mathrm{Fl}, \mathrm{Fr}$ & $6,7,8,9$ \\
\hline Casearia sanchezii J. Linares \& D. Angulo F. FMG7717 Chis, S & Á & $\mathrm{Au}$ & $\mathrm{Fl}$ & 3 \\
\hline Neopringlea viscosa (Liebm.) Rose DEB \& FSKs/n & Á & $\mathrm{Au}$ & - & 8 \\
\hline Olmediella betschleriana (Göpp.) Loes. JAEJ632 & $\mathrm{Ab}$ & $\mathrm{Au}$ & $\mathrm{E}$ & 2 \\
\hline Xylosma chlorantha Donn. Sm. GUR, EMS \& AM39 & $\mathrm{Ab}$ & $\mathrm{Au}$ & $\mathrm{Fr}$ & 2 \\
\hline $\begin{array}{l}\text { Xylosma flexuosa (Kunth) Hemsl. DEB30018, 33752, 33784, DEB } \\
\quad \text { \& FA48462, GUR39, JAEJ553 }\end{array}$ & $\mathrm{Ab}$ & $\mathrm{Au}$ & $\mathrm{Fr}$ & 2,12 \\
\hline Xylosma intermedia (Seem.) Triana \& Planch. DEB51509, DEB \& FSKs/n & Á & $\mathrm{Au}$ & - & 6,7 \\
\hline Xylosma velutina (Tul.) Triana \& Planch. DEB35076, 41550 & Á & $\mathrm{Au}$ & - & 5,11 \\
\hline Zuelania guidonia (Sw.) Britton \& Millsp. JAEJ759 & Á & $\mathrm{Au}$ & $\mathrm{Fl}$ & 4 \\
\hline \multicolumn{5}{|l|}{ Gentianaceae } \\
\hline Lisianthius nigrescens Schltdl. \& Cham. RORRs/n & $\mathrm{Ab}$ & $\mathrm{Au}$ & $\mathrm{Fl}$ & 5 \\
\hline $\begin{array}{l}\text { Lisianthus nigrescens var. chiapensis Cham. \& Schltdl. DEB14006, 27164, } \\
\quad \text { 41536, 51589, 69740, SDK77284, REW2168, PCLs/n }\end{array}$ & $\mathrm{Hi}$ & $\mathrm{Au}, \mathrm{Ru}$ & $\mathrm{Fl}, \mathrm{Fr}$ & $\begin{array}{l}4,7,8,9 \\
11,10\end{array}$ \\
\hline \multicolumn{5}{|l|}{ Gesneriaceae } \\
\hline Achimenes cettoana H.E. Moore JAEJ347 Chis & $\mathrm{Hi}$ & $\mathrm{Ru}$ & $\mathrm{Fl}$ & 9 \\
\hline Achimenes erecta (Lam.) H.P. Fuchs RTC, EC \& MHs/n & $\mathrm{Hi}$ & $\mathrm{Ru}$ & $\mathrm{Fl}$ & 10 \\
\hline Achimenes grandiflora (Schltdl.) DC. JAEJ317 & $\mathrm{Hi}$ & $\mathrm{Ru}$ & $\mathrm{Fl}$ & 9 \\
\hline Achimenes longiflora DC. JAEJ \& ALP255 Chis, C & $\mathrm{Hi}$ & $\mathrm{Ru}$ & $\mathrm{Fl}$ & 8 \\
\hline Achimenes misera Lindl. DEB \& FSKs/n, JAEJ322, 349 Chis, C & $\mathrm{Hi}$ & $\mathrm{Ru}$ & $\mathrm{Fl}$ & 8,9 \\
\hline Achimenes pedunculata Benth. JAEJ \& ALP283, JAEJ376, 378, 379 & $\mathrm{Hi}$ & $\mathrm{Ru}$ & $\mathrm{Fl}$ & 8 \\
\hline Besleria sp.1 JAEJ656 & $\mathrm{Hi}$ & $\mathrm{Ru}$ & $\mathrm{Fl}$ & 2 \\
\hline Diastema racemiferum Benth. MDRS35 & $\mathrm{Hi}$ & $\mathrm{Ru}$ & $\mathrm{FI}$ & 8 \\
\hline Episcia punctata (Lindl.) Hanst. & $\mathrm{Hi}$ & $\mathrm{Ru}$ & $\mathrm{Fl}$ & 8 \\
\hline $\begin{array}{l}\text { Kohleria rugata (Scheidw.) L.P. Kvist \& L.E. Skog. DEB13901, 27137, } \\
\text { 39936,52150, DEB \& FSKs/n, JAEJ348, JAEJ \& ALP253, DEB14687 }\end{array}$ & $\mathrm{Hi}$ & $\mathrm{Ru}$ & $\mathrm{Fl}$ & $8,9,10$ \\
\hline Moussonia deppeana (S. \& C.) Fritch. DEB \& ARS21582 & $\mathrm{Ab}$ & $\mathrm{Au}$ & - & 1 \\
\hline Moussonia elegans Decne. DEB \& ARS21582 & $\mathrm{Ab}$ & $\mathrm{Au}$ & $\mathrm{Fl}$ & 11 \\
\hline $\begin{array}{l}\text { Sinningia incarnata (Aubl.) D.L. Denham DEB14690, 27261ª, } \\
\text { 52179, JAEJ \& ALP252 }\end{array}$ & $\mathrm{Hi}$ & $\mathrm{Au}$ & $\mathrm{Fl}$ & 8 \\
\hline
\end{tabular}


Apéndice 1. Continuación

\begin{tabular}{|c|c|c|c|c|}
\hline Familia/Especie/ Colector & FC & FN & FE & MC \\
\hline \multicolumn{5}{|l|}{ Hernandiaceae } \\
\hline Gyrocarpus americanus Jacq. DEB13930 & Á & $\mathrm{Au}$ & $\mathrm{Fl}$ & 10 \\
\hline \multicolumn{5}{|l|}{ Hippocrateaceae } \\
\hline Hippocratea excelsa Kunth ARG524, JAEJ339, 645 & $\mathrm{Ab}$ & $\mathrm{Au}$ & $\mathrm{Fl}, \mathrm{Fr}$ & $2,5,9$ \\
\hline Hippocratea volubilis L. DEB35079 & $\mathrm{Ab}$ & $\mathrm{Au}$ & $\mathrm{Fl}$ & 5 \\
\hline \multicolumn{5}{|l|}{ Hydrophyllaceae } \\
\hline Nama dichotomum (Ruiz \& Pav.) Choisy DEB27165 & $\mathrm{Hi}$ & $\mathrm{Au}$ & $\mathrm{Fl}$ & 8 \\
\hline Wigandia urens (Ruiz \& Pav.) Kunth CHP3048, FMG108, JAEJ574 & $\mathrm{Ab}$ & $\mathrm{Au}$ & $\mathrm{Fl}$ & 1 \\
\hline \multicolumn{5}{|l|}{ Lamiaceae } \\
\hline $\begin{array}{l}\text { Catopheria chiapensis A. Gray ex Benth. DEB14013, DEB \& ARS21568, } \\
\text { DEB \& BB55479, DEB, ER \& PH13393, EMS, OT, MSS \& GD8585, } \\
\text { EMS, OT, MSS \& GDs/n, GD, MSS, OT, EMS \& JDs/n, JLPE } \\
\text { \& CCCs/n Chis, C }\end{array}$ & $\mathrm{Ab}$ & $\mathrm{Au}$ & - & $1,10,11$ \\
\hline Hyptis mutabilis (Rich.) Briq. AM95, JAEJ445 & $\mathrm{Hi}$ & $\mathrm{Au}$ & $\mathrm{Fl}$ & 2,10 \\
\hline Hyptis tomentosa Poit. DEB9025, RTC,CM \& GMs/n, SGS10 & $\mathrm{Hi}$ & $\mathrm{Au}$ & $\mathrm{Fl}$ & $2,4,5$ \\
\hline Hyptis urticoides Kunth DEB13924 & $\mathrm{Hi}$ & $\mathrm{Au}$ & - & 10 \\
\hline Hyptis verticillata Jacq. DEB52197 & $\mathrm{Ab}$ & $\mathrm{Au}$ & - & 8 \\
\hline Lepechinia schiedeana (Schltdl.) VatkeJMHJrs/n & $\mathrm{Hi}$ & $\mathrm{Au}$ & $\mathrm{Fl}$ & 9 \\
\hline Marsypianthes chamaedrys (Vahl) Kuntze DEB70056 & $\mathrm{Hi}$ & $\mathrm{Au}$ & - & 9 \\
\hline Ocimum micranthum Willd. DEB52189, JAEJ400 & $\mathrm{Hi}$ & $\mathrm{Au}$ & $\mathrm{Fr}$ & 8 \\
\hline Ocimum selloi Benth. AFS14 & $\mathrm{Ab}$ & $\mathrm{Au}$ & $\mathrm{Fl}$ & 3 \\
\hline Salvia albiflora M. Martens \& Galeotti DEB41552, 69727 & $\mathrm{Hi}$ & $\mathrm{Au}$ & $\mathrm{Fl}$ & 9,11 \\
\hline Salvia amarissima Ortega DEB27161 & $\mathrm{Hi}$ & $\mathrm{Au}$ & $\mathrm{FI}$ & 8 \\
\hline Salvia chiapensis Fernald DEB27266, HME454 Chis & $\mathrm{Hi}$ & $\mathrm{Au}$ & $\mathrm{FI}$ & 6 \\
\hline Salvia cinnabarina M. Martens \& Galeotti JAEJ241 & $\mathrm{Hi}$ & $\mathrm{Au}$ & $\mathrm{FI}$ & 7 \\
\hline Salvia coccinea Buc'hoz ex Etl. DEB70018, JAEJ771 & $\mathrm{Hi}$ & $\mathrm{Au}$ & $\mathrm{FI}$ & 6,9 \\
\hline Salvia lasiantha Benth. CRB188, EMS14271, JAEJ523 & $\mathrm{Ab}$ & $\mathrm{Au}$ & $\mathrm{FI}$ & $9,10,11$ \\
\hline Salvia lavanduloides Kunth JAEJ475 & $\mathrm{Hi}$ & $\mathrm{Au}$ & $\mathrm{FI}$ & 10 \\
\hline Salvia longispicata M. Martens \& Galeotti JAEJ146 & $\mathrm{Hi}$ & $\mathrm{Au}$ & $\mathrm{Fl}$ & 7 \\
\hline Salvia miniata Fernald DEB10670, 27153, 28172, DEB \& FSKs/n Be, Chis, G & $\mathrm{Hi}$ & $\mathrm{Au}$ & $\mathrm{FI}$ & $7,8,9$ \\
\hline Salvia misella Kunth AM, GUR \& EMS107-bis, DEB41493 & $\mathrm{Hi}$ & $\mathrm{Au}$ & $\mathrm{FI}$ & 2,11 \\
\hline Salvia occidentalis Sw. AM, GUR \& EMS107 & $\mathrm{Hi}$ & $\mathrm{Au}$ & - & 2 \\
\hline Salvia polystachya Ortega EMSs/n, JAEJ510, TLW, EJLTT \& JGPs/n & $\mathrm{Hi}$ & $\mathrm{Au}$ & $\mathrm{FI}$ & 11,12 \\
\hline Salvia purpurea Cav. JAEJ454 & $\mathrm{Hi}$ & $\mathrm{Au}$ & $\mathrm{FI}$ & 10 \\
\hline Salvia tiliaefolia Vahl ARGs/n & $\mathrm{Hi}$ & $\mathrm{Au}$ & - & 11 \\
\hline Salvia tonalensis Brandegee DEB52188 & $\mathrm{Hi}$ & $\mathrm{Au}$ & - & 8 \\
\hline Scutellaria inflata Epling DEB39998 & $\mathrm{Hi}$ & $\mathrm{Au}$ & - & 9 \\
\hline Scutellaria seleriana Loes. DEB27174, DEB \& BB55481, DEB, ER \& PH13383 & $\mathrm{Hi}$ & $\mathrm{Au}$ & - & $8,10,11$ \\
\hline \multicolumn{5}{|l|}{ Lauraceae } \\
\hline Cassytha filiformis L. DEB10676 & $\mathrm{Li}$ & $\mathrm{Pa}$ & - & 7 \\
\hline Cinnamomum triplinerve (Ruiz \& Pav.) Kosterm. JAEJ746 & Á & $\mathrm{Au}$ & - & 4 \\
\hline Licaria caudata (Lundell) Kosterm. JAEJ98, 160, 723 & Á & $\mathrm{Au}$ & $\mathrm{Fr}$ & $4,6,7$ \\
\hline Litsea glaucescens Kunth JAEJ636 P, Chis, C & Á & $\mathrm{Au}$ & $\mathrm{FI}$ & 2 \\
\hline Ocotea tonii (Lundell) van der Werff DEB70059 & Á & $\mathrm{Au}$ & - & 9 \\
\hline Phoebe fruticosa Lundell DEB10671 & Á & $\mathrm{Au}$ & - & 7 \\
\hline \multicolumn{5}{|l|}{ Lentibulariaceae } \\
\hline Pinguicula moranensis Kunth DEB \& ARS21586, JAEJ630 & $\mathrm{Hi}$ & $\mathrm{Ru}$ & $\mathrm{Fl}$ & 2,11 \\
\hline \multicolumn{5}{|l|}{ Linaceae } \\
\hline Linum rupestre (A. Gray) Engelm. ex A. Gray DEB20163, 37220, 41537 & $\mathrm{Hi}$ & $\mathrm{Au}$ & - & $9,10,11$ \\
\hline
\end{tabular}


Apéndice 1. Continuación

\begin{tabular}{|c|c|c|c|c|}
\hline Familia/Especie/ Colector & FC & FN & FE & MC \\
\hline \multicolumn{5}{|l|}{ Loasaceae } \\
\hline Gronovia scandens L. JAEJ \& ALP263 & $\mathrm{Li}$ & $\mathrm{Au}$ & $\mathrm{Fl}$ & 8 \\
\hline $\begin{array}{l}\text { Mentzelia hispida Willd. DEB13900, 39923, DEB \& ARS21571, } \\
\text { DEB \& FA48467 }\end{array}$ & $\mathrm{Hi}$ & $\mathrm{Au}$ & - & $9,10,11,12$ \\
\hline \multicolumn{5}{|l|}{ Loganiaceae } \\
\hline Buddleja americana L. ARG, RH \& PJSs/n & $\mathrm{Ab}$ & $\mathrm{Au}$ & - & 1 \\
\hline Buddleja nitida Benth. JMHJrs/n & $\mathrm{Ab}$ & $\mathrm{Au}$ & - & 9 \\
\hline Spigelia humboldtiana Cham. \& Schltdl DEB14689,40005, JAEJ123, 377 & $\mathrm{Hi}$ & $\mathrm{Au}$ & $\mathrm{Fl}$ & $6,8,9$ \\
\hline Spigelia pygmaea D.N. Gibson DEB39969 & $\mathrm{Hi}$ & $\mathrm{Au}$ & - & 9 \\
\hline $\begin{array}{l}\text { Spigelia splendens H. Wendl. ex Hook. DEB10643, DEB \& FSKs/n, } \\
\text { FMG7807, JAEJ107, JLPE, JIC \& CCCs/n }\end{array}$ & $\mathrm{Hi}$ & $\mathrm{Au}$ & - & 6,7 \\
\hline \multicolumn{5}{|l|}{ Loranthaceae } \\
\hline Psittacanthus calyculatus (DC.) G. Don DEBs/n, JAEJ \& ALP268, RMKs/n & $\mathrm{Hi}$ & $\mathrm{Pa}$ & $\mathrm{Fl}$ & $6,8,10$ \\
\hline Psittacanthus mayanus Standl. \& Steyerm. DEB14017 & $\mathrm{Hi}$ & $\mathrm{Pa}$ & - & 10 \\
\hline Struthanthus deppeanus (Schltdl. \& Cham.) D. Don GUR28 & $\mathrm{Hi}$ & $\mathrm{Pa}$ & $\mathrm{Fl}$ & 2 \\
\hline Struthanthus quadrangularis Kuijt JAEJ487 & $\mathrm{Hi}$ & $\mathrm{Pa}$ & $\mathrm{Fr}$ & 10 \\
\hline Struthanthus quercicola (Schltdl. \& Cham.) Blume DEB, ER \& PH14018 & $\mathrm{Hi}$ & $\mathrm{Pa}$ & - & 10 \\
\hline Struthanthus tacanensis Lundell DEBs/n & $\mathrm{Hi}$ & $\mathrm{Pa}$ & - & 10 \\
\hline \multicolumn{5}{|l|}{ Lythraceae } \\
\hline Cuphea aequipetala Cav. JMHJrs/n, JAEJ469 & $\mathrm{Hi}$ & $\mathrm{Au}$ & $\mathrm{Fl}$ & 9,10 \\
\hline Cuphea appendiculata Benth. DEB10667, 51510, DEB \& FSKs/n & $\mathrm{Hi}$ & $\mathrm{Au}$ & $\mathrm{Fl}$ & 7 \\
\hline Cuphea carthagenensis (Jacq.) J.F. Macbr. FMG5490 & $\mathrm{Hi}$ & $\mathrm{Au}$ & $\mathrm{FI}$ & 9 \\
\hline Cuphea hookeriana Walp. JAEJ130 & $\mathrm{Hi}$ & $\mathrm{Au}$ & $\mathrm{Fl}$ & 6 \\
\hline Cuphea hyssopifolia Kunth JAEJ83, 598 & $\mathrm{Hi}$ & $\mathrm{Au}$ & $\mathrm{Fl}$ & 1,6 \\
\hline Cuphea ignea A. DC. DEB \& BB55480 & $\mathrm{Hi}$ & $\mathrm{Au}$ & $\mathrm{Fl}$ & 11 \\
\hline Cuphea intermedia HemsI. DEB14008, 28175, 52199, DEB \& FSKs/n & $\mathrm{Hi}$ & $\mathrm{Au}$ & $\mathrm{Fl}$ & $8,9,10$ \\
\hline Cuphea leptopoda Hemsl. DEB10634, 14692, 51517, 52143, DEB \& FSKs/n & $\mathrm{Hi}$ & $\mathrm{Au}$ & $\mathrm{FI}$ & 7,8 \\
\hline Cuphea lutea Rose DEB, ER \& PH13506 & $\mathrm{Hi}$ & $\mathrm{Au}$ & $\mathrm{Fl}$ & 10 \\
\hline Cuphea nitidula Kunth CRB192 & $\mathrm{Hi}$ & $\mathrm{Au}$ & $\mathrm{Fl}$ & 9 \\
\hline Cuphea wrightii subsp. wrightii DEB51694 & $\mathrm{Hi}$ & $\mathrm{Au}$ & - & 7 \\
\hline Ginoria nudiflora (Hemsl.) Koehne DEB35078, 50647, 51661, MGRG109 & Á & $\mathrm{Au}$ & - & $4,5,7$ \\
\hline \multicolumn{5}{|l|}{ Malpighiaceae } \\
\hline Bunchosia biocellata Schltdl. JAEJ724 & $\mathrm{Ab}$ & $\mathrm{Au}$ & $\mathrm{Fr}$ & 4 \\
\hline $\begin{array}{l}\text { Byrsonima crassifolia (L.) Kunth ARG522, DCSa, DEB \& FSKs/n, } \\
\text { JAEJ189, JHCs/n, SGS9 }\end{array}$ & Á & $\mathrm{Au}$ & $\mathrm{Fl}, \mathrm{Fr}$ & $3,4,5,7$ \\
\hline Callaeum malpighioides (Turcz.) D.M. Johnson DEB \& BB55469 & $\mathrm{Li}$ & $\mathrm{Au}$ & - & 11 \\
\hline Galphimia glauca Cav. DEB13911, JAEJ504, EC5957 & $\mathrm{Ab}$ & $\mathrm{Au}$ & $\mathrm{FI}$ & 10,11 \\
\hline $\begin{array}{l}\text { Gaudichaudia albida Schltdl. \& Cham. WRA \& ChWL4222, } \\
\text { WRA13236, JAEJ511 }\end{array}$ & $\mathrm{Li}$ & $\mathrm{Au}$ & $\mathrm{FI}$ & 10,11 \\
\hline Heteropterys brachiata (L.) DC. DEB27182, JAEJ \& ALP308 & $\mathrm{Li}$ & $\mathrm{Au}$ & $\mathrm{FI}$ & 8 \\
\hline Heteropterys cotinifolia A. Juss. DEB39953 & $\mathrm{Li}$ & $\mathrm{Au}$ & - & 9 \\
\hline Heteropterys laurifolia (L.) A. Juss. RGA527 & $\mathrm{Li}$ & $\mathrm{Au}$ & - & 5 \\
\hline Hiraea fagifolia (DC.) A. Juss. JAEJ713 & $\mathrm{Li}$ & $\mathrm{Au}$ & $\mathrm{Fr}$ & 3 \\
\hline Malpighia glabra L. DEB \& ARS21579, DEB10658, DFG47, JAEJ402 & $\mathrm{Ab}$ & $\mathrm{Au}$ & $\mathrm{Fl}, \mathrm{Fr}$ & $7,8,10,11$ \\
\hline Mascagnia nicaraguensis (Griseb.) Niedenzu GUR30 & Á & $\mathrm{Au}$ & $\mathrm{Fr}$ & 2 \\
\hline $\begin{array}{l}\text { Psychopterys multiflora (Nied.) W.R. Anderson \& S. Corso EM22323, } \\
\text { DN5519 Chis, G, Oax }\end{array}$ & $\mathrm{Li}$ & $\mathrm{Au}$ & $\mathrm{Fl}$ & - \\
\hline $\begin{array}{l}\text { Stigmaphyllon ellipticum (Kunth) A. Juss. LFMJ3, JAEJ465, } \\
\text { 610, LFMJ3, RCC21 }\end{array}$ & $\mathrm{Hi}, \mathrm{Li}$ & $\mathrm{Au}$ & $\mathrm{Fl}$ & $2,5,10$ \\
\hline Stigmaphyllon lindenianum A. Juss. AM, GUR \& EMS30, AM,GUR \& EMS81 & $\mathrm{Li}$ & $\mathrm{Au}$ & $\mathrm{Fl}, \mathrm{Fr}$ & 2 \\
\hline
\end{tabular}


Apéndice 1. Continuación

\begin{tabular}{|c|c|c|c|c|}
\hline Familia/Especie/ Colector & FC & FN & FE & MC \\
\hline Stigmaphyllon selerianum Nied. DEB9027 & $\mathrm{Li}$ & $\mathrm{Au}$ & - & 2 \\
\hline Tetrapterys heterophylla (Griseb.) W.R. Anderson DEB9028 & $\mathrm{Li}$ & $\mathrm{Au}$ & - & 2 \\
\hline Tetrapterys schiedeana Schltdl. \& Cham. DEB14405, DEB70047, DEB51957 & $\mathrm{Li}$ & $\mathrm{Au}$ & - & $7,8,9$ \\
\hline \multicolumn{5}{|l|}{ Malvaceae } \\
\hline Abutilon purpusii Standl. DEB13886 & $\mathrm{Ab}$ & $\mathrm{Au}$ & - & 10 \\
\hline Anoda acerifolia Cav. JAEJ \& ALP299 & $\mathrm{Hi}$ & $\mathrm{Au}$ & $\mathrm{Fl}$ & 8 \\
\hline Anoda cristata (L.) Schltdl. JAEJ443 & $\mathrm{Hi}$ & $\mathrm{Au}$ & $\mathrm{Fr}$ & 10 \\
\hline \multicolumn{5}{|l|}{ \& DEB20124 Chis, Oax, Ver } \\
\hline Bakeridesia integerrima (Hook. f.) D.M. Bates DN5522 & - & $\mathrm{Au}$ & - & 3 \\
\hline Bakeridesia nelsonii (Rose) D.M. Bates DEB9044 & - & $\mathrm{Au}$ & - & 2 \\
\hline Bakeridesia pittieri (Donn. Sm.) D.M. Bates DEB9022 & $\mathrm{Ab}$ & $\mathrm{Au}$ & - & 2 \\
\hline Bastardiastrum incanum (Brandegee) D.M. Bates ARGs/n & $\mathrm{Ab}$ & $\mathrm{Au}$ & - & 11 \\
\hline $\begin{array}{l}\text { Dendrosida sharpiana (Miranda) Fryxell CRB1258, 356, DEB \& FSKs/n, } \\
\text { GD, MSS, OT, EMS \& JDs/n, PAF \& EJLTTs/n, PAF \& REMs/n }\end{array}$ & $\mathrm{Ab}$ & $\mathrm{Au}$ & $\mathrm{FI}$ & $9,10,11$ \\
\hline & \multicolumn{3}{|c|}{ DEB70040, GD. Et al29725, JAEJ319, JBZ9 Chis } & $9,10,11$ \\
\hline Gaya minutiflora Rose DEB52001, DEB52182 & $\mathrm{Hi}$ & $\mathrm{Au}$ & - & 8 \\
\hline Gossypium hirsutum L. ACH1 & $\mathrm{Hi}$ & $\mathrm{Au}$ & $\mathrm{Fl}$ & 11 \\
\hline Hampea mexicana Fryxell PAF \& DMB900 & Á & $\mathrm{Au}$ & $\mathrm{Fl}$ & 1 \\
\hline Hampea stipitata S. Watson. JAEJ693 & Á & $\mathrm{Au}$ & $\mathrm{Fl}$ & 3 \\
\hline Herissantia crispa (L.) Brizicky AM, GUR \& EMS40 & $\mathrm{Hi}$ & $\mathrm{Au}$ & $\mathrm{Fl}$ & 2 \\
\hline Hibiscus lavateroides Moric. ex Ser. DLA22 & $\mathrm{Ab}$ & $\mathrm{Au}$ & $\mathrm{Fl}$ & 5 \\
\hline Hibiscus poeppigii (Spreng.) Garcke DN5500, JAEJ \& JMM192 & $\mathrm{Ab}$ & $\mathrm{Au}, \mathrm{Ru}$ & $\mathrm{Fl}$ & 3,7 \\
\hline Malvastrum corchorifolium (Desr.) Britton ex Small. DEB10655 & $\mathrm{Hi}$ & $\mathrm{Au}$ & - & 7 \\
\hline Malvastrum coromandelianum (L.) Garcke. DEB10655 & $\mathrm{Hi}$ & $\mathrm{Au}$ & - & 7 \\
\hline Malvaviscus arboreus Cav. AG \& AMC6, JAEJ \& ALP275 & $\mathrm{Ab}$ & $\mathrm{Au}$ & $\mathrm{FI}$ & 1,8 \\
\hline Malvaviscus arboreus var. arboreus Cav. DEB10654, JAEJ334 & $\mathrm{Ab}$ & $\mathrm{Au}$ & $\mathrm{Fl}, \mathrm{Fr}$ & 7,9 \\
\hline Pavonia schiedeana Steud. DEB51659 & $\mathrm{Hi}$ & $\mathrm{Au}$ & - & 7 \\
\hline Pseudabutilon scabrum (C. Presl) R.E. Fr. DEB \& JLS47034, PAF3236 & $\mathrm{Ab}$ & $\mathrm{Au}$ & - & 10 \\
\hline Robinsonella lindeniana subsp. divergens (Rose \& Baker f.) Fryxell JAEJ541 & Á & $\mathrm{Au}$ & $\mathrm{Fl}$ & 12 \\
\hline Robinsonella mirandae Gómez Pompa MGVM25 & $\mathrm{Ab}$ & $\mathrm{Au}$ & $\mathrm{Fl}$ & 3 \\
\hline Sida abutifolia Mill. DEBs/n & $\mathrm{Hi}$ & $\mathrm{Au}$ & $\mathrm{FI}$ & 11 \\
\hline Sida acuta Burm. F. JMHJrs/n & $\mathrm{Ab}$ & $\mathrm{Au}$ & $\mathrm{Fl}$ & 9 \\
\hline Sida glutinosa Comm. ex Cav. EMS, OT, MSS \& GDs/n & $\mathrm{Hi}$ & $\mathrm{Au}$ & $\mathrm{Fl}$ & 11 \\
\hline Sida rhombifolia L. DLA34 & $\mathrm{HI}$ & $\mathrm{Au}$ & $\mathrm{FI}$ & 5 \\
\hline Sida spinosa L. DEB26222 & $\mathrm{Hi}$ & $\mathrm{Au}$ & $\mathrm{FI}$ & 7 \\
\hline Sida urens L. JAEJ \& ALP297 & $\mathrm{Ab}$ & $\mathrm{Au}$ & $\mathrm{Fl}$ & 8 \\
\hline \multicolumn{5}{|l|}{ Melastomataceae } \\
\hline Arthrostemma ciliatum Pav. ex D. Don VAGL30 & $\mathrm{Hi}$ & $\mathrm{Au}$ & $\mathrm{Fl}$ & 11 \\
\hline Conostegia xalapensis (Bonpl.) D. Don ex DC. RMC623, RMC630 & $\mathrm{Ab}$ & $\mathrm{Au}$ & $\mathrm{Fl}$ & 8 \\
\hline Heterocentron muricatum Gleason DEB70254 Chis & $\mathrm{Hi}$ & $\mathrm{Au}$ & - & 9 \\
\hline $\begin{array}{l}\text { Heterocentron subtriplinervium (Link \& Otto) A. Braun \& C.D. } \\
\text { Bouché JAEJ \& ALP289 }\end{array}$ & $\mathrm{Hi}$ & $\mathrm{Au}$ & $\mathrm{FI}$ & 8 \\
\hline Miconia guatemalensis Cogn. JAEJ481 & $\mathrm{Hi}$ & $\mathrm{Au}$ & $\mathrm{Fr}$ & 10 \\
\hline Miconia laevigata (L.) D. Don DEB51511 & $\mathrm{Ab}$ & $\mathrm{Au}$ & - & 7 \\
\hline Miconia sylvatica (Schltdl.) Naudin DEB70033 & $\mathrm{Ab}$ & $\mathrm{Au}$ & - & 9 \\
\hline Topobea laevigata (D. Don) Naudin JM10 & Á & $\mathrm{Au}$ & - & 11 \\
\hline \multicolumn{5}{|l|}{ Meliaceae } \\
\hline Cedrela oaxacensis C. DC. \& Rose ER, PRH, DEB. 20139 & Á & $\mathrm{Au}$ & - & 1 \\
\hline
\end{tabular}


Apéndice 1. Continuación

\begin{tabular}{|c|c|c|c|c|}
\hline Familia/Especie/ Colector & FC & FN & FE & MC \\
\hline Cedrela odorata L. AM, GUR \& EMS98, JAEJ149, 353, 578 Pr & Á & $\mathrm{Au}$ & $\mathrm{Fl}, \mathrm{Fr}$ & $1,2,7,9$ \\
\hline Cedrela salvadorensis Standl. EUVP5, MDRS5 & Á & $\mathrm{Au}$ & $\mathrm{Fr}$ & 8,10 \\
\hline Swietenia humilis Zucc. DN5523 & Á & $\mathrm{Au}$ & - & 3 \\
\hline Trichilia hirta L. JAEJ183 & Á & $\mathrm{Au}$ & $\mathrm{Fl}$ & 7 \\
\hline \multicolumn{5}{|l|}{ Menispermaceae } \\
\hline Abuta chiapasensis Krukoff \& Barneby FMG7757, 7757A Chis & $\mathrm{Li}$ & $\mathrm{Au}$ & $\mathrm{Fl}$ & 4 \\
\hline Cissampelos pareira L. AM, GUR \& EMS73, JAEJ104, 781, PTL,JL \& ARRs/n & $\mathrm{Li}$ & $\mathrm{Au}$ & $\mathrm{Fl}, \mathrm{Fr}$ & $1,2,3,6$ \\
\hline $\begin{array}{l}\text { Hyperbaena mexicana Miers. FMG7757, JFL36, JAMA7, JMM, et al. } \\
\text { 1741, RO, JP, JMM \& JLCHs/n }\end{array}$ & Á & $\mathrm{Au}$ & - & 1,2 \\
\hline \multicolumn{5}{|l|}{ Moraceae } \\
\hline Dorstenia contrajerva L. AML19CEMM17, DEB70273, JAEJ236, OFS1062 & $\mathrm{Hi}$ & $\mathrm{Au}$ & $\mathrm{Fl}, \mathrm{Fr}$ & $1,7,9,10$ \\
\hline Dorstenia drakena L. JAEJ \& JMM202 & $\mathrm{Hi}$ & $\mathrm{Au}$ & $\mathrm{Fl}$ & 7 \\
\hline Ficus aurea Nutt. DEB50650, OFC232, DEB70260, DEB51998 & Á & $\mathrm{Au}$ & $\mathrm{Fr}$ & $4,8,9$ \\
\hline Ficus cooki Standl. ARG934, FDAL27, JAEJ133 Chis, G, Ver & Á & $\mathrm{Au}$ & $\mathrm{Fr}$ & $6,9,10$ \\
\hline Ficus cotinifolia Kunth JAEJ74 & Á & $\mathrm{Au}$ & $\mathrm{Fr}$ & 6 \\
\hline Ficus isophlebia Standl. RTC, EC \& MHs/n & Á & $\mathrm{Au}$ & $\mathrm{Fr}$ & 10 \\
\hline Ficus maxima Mill. DEB51947 & Á & $\mathrm{Au}$ & $\mathrm{Fr}$ & 8 \\
\hline Ficus obtusifolia Kunth JAEJ231, 405, 517 & Á & $\mathrm{Au}$ & $\mathrm{Fr}$ & $7,8,11$ \\
\hline $\begin{array}{l}\text { Ficus pertusa L. f. DEB51965, DEB48476, DEB \& Fas/n, JAEJ230, } \\
\quad 356,622,696, \text { RTC, EC \& MH }\end{array}$ & Á & $\mathrm{Au}$ & $\mathrm{Fr}$ & $\begin{array}{l}2,3,7,8 \\
9,10,12\end{array}$ \\
\hline Ficus trigonata L. DEB70038 & Á & $\mathrm{Au}$ & $\mathrm{Fr}$ & 9 \\
\hline \multicolumn{4}{|l|}{ Myricaceae } & $2,4,9$ \\
\hline Morella cerifera (L.) Small. JAEJ474, 477, 478 & $\mathrm{Ab}$ & $\mathrm{Au}$ & $\mathrm{Fl}, \mathrm{Fr}$ & 10 \\
\hline \multicolumn{5}{|l|}{ Myrsinaceae } \\
\hline Ardisia compressa Kunth. GU \& EMS \& AMG11, JAEJ627 & Á & $\mathrm{Au}$ & $\mathrm{Fr}$ & 2 \\
\hline Ardisia densiflora Krug \& Urb. DEB51678, FMG7659 & $\mathrm{Ab}$ & $\mathrm{Au}$ & $\mathrm{Fl}, \mathrm{Fr}$ & 1,7 \\
\hline $\begin{array}{l}\text { Ardisia escallonioides Schltdl. \& Cham. CRB193, DEB13928, 2155, } \\
\quad \text { DEB \& FSKs/n, GUR \& EMS \& AMG11-bis }\end{array}$ & $\mathrm{Ab}$ & $\mathrm{Au}$ & $\mathrm{Fl}, \mathrm{Fr}$ & $2,8,9,10$ \\
\hline Parathesis breedlovei Lundell. JAEJ513 Chis & Á & $\mathrm{Au}$ & $\mathrm{Fr}$ & 11 \\
\hline Parathesis nigropunctata Lundell. GUR11A & Á & $\mathrm{Au}$ & $\mathrm{Fr}$ & 2 \\
\hline Parathesis rubriflora Lundell. DEB51660, DEB \& FSKs/n & - & - & - & 7 \\
\hline Parathesis vulgata Lundell. JAEJ753 & $\mathrm{Hi}$ & $\mathrm{Au}$ & $\mathrm{Fl}$ & 4 \\
\hline \multicolumn{5}{|l|}{ Myrtaceae } \\
\hline Calyptranthes chiapensis Lundell JAEJ77, 80, 726 Chis, Ver & $\mathrm{Ab}$ & $\mathrm{Au}, \mathrm{Ru}$ & $\mathrm{Fl}, \mathrm{Fr}$ & 4,6 \\
\hline Eugenia acapulcensis Steud. AM, GUR \& EMS90, JAEJ626 & $\mathrm{Ab}$ & $\mathrm{Au}$ & $\mathrm{Fr}$ & 2 \\
\hline Eugenia breedlovei Barrie DRG107 Chis & Á & $\mathrm{Au}$ & $\mathrm{Fr}$ & 4 \\
\hline $\begin{array}{l}\text { Eugenia capuli (Schltdl. \& Cham.) Hook. \& Arn. DEB13927, 27145, } \\
\text { DNs/n, JAEJ124, } 506\end{array}$ & Á & $\mathrm{Au}$ & $\mathrm{Fl}, \mathrm{Fr}$ & $\begin{array}{l}1,3,6 \\
10,11\end{array}$ \\
\hline Eugenia galalonensis (C. Wright ex Griseb.) Krug \& Urb. EMS et al.8584 & Á & $\mathrm{Au}$ & - & 11 \\
\hline Eugenia guatemalensis Donn. Sm. DEB13296, DEB \& FSKs/n & Á, $\mathrm{Ab}$ & $\mathrm{Au}$ & - & 10 \\
\hline Eugenia oerstediana O. Berg JAEJ522 & $\mathrm{Ab}$ & $\mathrm{Au}$ & $\mathrm{Fr}$ & 11 \\
\hline Eugenia rhombea Krug \& Urb. DEB49062 & Á & $\mathrm{Au}$ & - & 1 \\
\hline $\begin{array}{l}\text { Eugenia siltepecana Lundell DEB13859, 14409, 26229, 27144, 51956, } \\
\text { DEB \& FSKs/n Chis }\end{array}$ & Á & $\mathrm{Au}$ & $\mathrm{Fl}, \mathrm{Fr}$ & $1,7,8,10$ \\
\hline Eugenia xalapensis (Kunth) DC. JAEJ112 & Á & $\mathrm{Au}$ & - & 6 \\
\hline Myrcianthes fragrans (Sw.) McVaugh GD29713, JAEJ742 & $\mathrm{Ab}$ & $\mathrm{Au}$ & $\mathrm{Fl}, \mathrm{Fr}$ & 4,11 \\
\hline Psidium guajava L. EUVP16, RML917 & Á & $\mathrm{Au}$ & $\mathrm{Fr}$ & 5,10 \\
\hline Psidium molle Bertol. JAEJ \& ALP271 & $\mathrm{Ab}$ & $\mathrm{Au}$ & $\mathrm{Fr}$ & 8 \\
\hline Syzygium jambos (L.) Alston JAEJ629 & Á & $\mathrm{Au}$ & $\mathrm{Fl}$ & 2 \\
\hline
\end{tabular}


Apéndice 1. Continuación

\begin{tabular}{|c|c|c|c|c|}
\hline Familia/Especie/ Colector & FC & FN & FE & MC \\
\hline \multicolumn{5}{|l|}{ Nyctaginaceae } \\
\hline Boerhavia diffusa L. DDLO40, DEB20192, JAEJ171 & $\mathrm{Hi}, \mathrm{Li}$ & $\mathrm{Au}$ & $\mathrm{FI}$ & $7,10,11$ \\
\hline Mirabilis jalapa L. JAEJ594 & $\mathrm{Hi}$ & $\mathrm{Au}$ & $\mathrm{FI}$ & 1 \\
\hline Mirabilis violaceae (L.) Heimerl DLA23 & $\mathrm{Hi}$ & $\mathrm{Au}$ & $\mathrm{FI}$ & 5 \\
\hline Neea psychotrioides Donn. Sm JAEJ777 & Á & $\mathrm{Au}$ & $\mathrm{Fr}$ & 6 \\
\hline Neea tenuis Standl. JAEJ316 Chis, Hon, Ver & $\mathrm{Ab}$ & $\mathrm{Au}$ & $\mathrm{Fr}$ & 9 \\
\hline Pisonia aculeata L. DEB30027, GUR44,58, JAEJ387, OFS999 & $\mathrm{Hi}$ & $\mathrm{Au}$ & $\mathrm{Fl}, \mathrm{Fr}$ & $1,2,11$ \\
\hline \multicolumn{5}{|l|}{ Ochnaceae } \\
\hline Ouratea tuerckheimii Donn. Sm. JAEJ603, 725 & $\mathrm{Ab}$ & $\mathrm{Au}$ & $\mathrm{Fl}, \mathrm{Fr}$ & 1,4 \\
\hline \multicolumn{5}{|l|}{ Olacaceae } \\
\hline Ximenia americana L. DCS33 & $\mathrm{Ab}$ & $\mathrm{Au}$ & $\mathrm{Fl}$ & 3 \\
\hline \multicolumn{5}{|l|}{ Oleaceae } \\
\hline $\begin{array}{l}\text { Fraxinus dubia (Willd. ex Schult. \& Schult. F.) P.S. Green \& M. Nee } \\
\text { ARG1456, DEB39967 }\end{array}$ & Á & $\mathrm{Au}$ & $\mathrm{FI}$ & 1,9 \\
\hline \multicolumn{5}{|l|}{ Onagraceae } \\
\hline Fuchsia thymifolia Kunth JMHJr27 & $\mathrm{Ab}$ & $\mathrm{Au}$ & - & 7 \\
\hline Gongylocarpus rubricaulis Schltdl. \& Cham. DEB13918 & $\mathrm{Hi}$ & $\mathrm{Au}$ & - & 10 \\
\hline Hauya elegans DC. JAEJ670, JAEJ \& ALP251, NMM794 & Á, $\mathrm{Ab}$ & $\mathrm{Au}$ & $\mathrm{Fr}$ & $1,5,8$ \\
\hline $\begin{array}{l}\text { Hauya elegans subsp. barcenae (Hemsl.) P.H. Raven \& Breedlove DEB } \\
\text { 10641, 10649, 10675, 13834, DEB, ER \& PH13540, JAEJ121 Chis, G }\end{array}$ & Á & $\mathrm{Au}$ & $\mathrm{Fl}, \mathrm{Fr}$ & $6,7,10$ \\
\hline $\begin{array}{l}\text { Hauya elegans subsp. cornuta (Hemsl.) P.H. Raven \& Breedlove } \\
\text { DEB35070 Chis, C }\end{array}$ & Á & $\mathrm{Au}$ & - & 5 \\
\hline Hauya heydeana Donn. Sm. DEB \& ARS21588 Chis, G & Á & $\mathrm{Au}$ & - & 11 \\
\hline Lopezia langmaniae Miranda DEB14016, 30019 Chis, G & $\mathrm{Ab}$ & $\mathrm{Au}$ & - & 10,12 \\
\hline Oenothera epilobiifolia Kunth JMHJr14 & $\mathrm{Hi}$ & $\mathrm{Au}$ & $\mathrm{Fl}$ & 10 \\
\hline \multicolumn{5}{|l|}{ Oxalidaceae } \\
\hline Oxalis corniculata L. OT \& JLVR6687 & $\mathrm{Hi}$ & $\mathrm{Au}$ & $\mathrm{Fl}$ & 1 \\
\hline Oxalis frutescens L. DEB51514 & $\mathrm{Hi}$ & $\mathrm{Au}$ & $\mathrm{Fl}$ & 7 \\
\hline $\begin{array}{l}\text { Oxalis frutescens ssp. angustifolia (Kunth) LourAuig JAEJ122, 235, } \\
\text { JAEJ \& ALP270 }\end{array}$ & $\mathrm{Hi}$ & $\mathrm{Au}$ & $\mathrm{FI}$ & $6,7,8$ \\
\hline Oxalis microcarpa Benth. DEB10669 & $\mathrm{Hi}$ & $\mathrm{Au}$ & $\mathrm{Fl}$ & 7 \\
\hline Oxalis nelsonii (Small) R. Knuth. DEB51685 & $\mathrm{Hi}$ & $\mathrm{Au}$ & $\mathrm{Fl}$ & 7 \\
\hline Oxalis rhombifolia Jacq. JAEJ165 & $\mathrm{Hi}$ & $\mathrm{Au}$ & $\mathrm{FI}$ & 7 \\
\hline Oxalis tetraphylla Cav. JAEJ770 & $\mathrm{Hi}$ & $\mathrm{Au}$ & $\mathrm{FI}$ & 6 \\
\hline \multicolumn{5}{|l|}{ Papaveraceae } \\
\hline Argemone mexicana L. AC11 & $\mathrm{Hi}$ & $\mathrm{Au}$ & $\mathrm{FI}$ & 12 \\
\hline Bocconia arborea S. Watson CZ13 & $\mathrm{Ab}$ & $\mathrm{Au}$ & $\mathrm{Fr}$ & 5 \\
\hline Bocconia frutescens L. JMHJr21 & $\mathrm{Ab}$ & $\mathrm{Au}$ & - & 6 \\
\hline \multicolumn{5}{|l|}{ Passifloraceae } \\
\hline Passiflora biflora Lam. DEB \& FA48470 & $\mathrm{Li}$ & $\mathrm{Au}$ & $\mathrm{FI}$ & 12 \\
\hline Passiflora foetida L. DTR26, MCLP18 & $\mathrm{Li}$ & $\mathrm{Au}$ & $\mathrm{FI}$ & 3,5 \\
\hline Passiflora guatemalensis S. Watson JAEJ363 & $\mathrm{Li}$ & $\mathrm{Au}$ & $\mathrm{FI}$ & 9 \\
\hline Passiflora oerstedii Mast. MARR16 & $\mathrm{Li}$ & $\mathrm{Au}$ & $\mathrm{FI}$ & 12 \\
\hline Passiflora standleyi Killip DEB37173 & $\mathrm{Li}$ & $\mathrm{Au}$ & $\mathrm{FI}$ & 9 \\
\hline Passiflora suberosa L. DEB51972, 52183 & $\mathrm{Li}$ & $\mathrm{Au}$ & $\mathrm{FI}$ & 8 \\
\hline \multicolumn{5}{|l|}{ Phytolaccaceae } \\
\hline Phytolacca icosandra L. JAEJ687 & $\mathrm{Hi}$ & $\mathrm{Au}$ & $\mathrm{Fl}, \mathrm{Fr}$ & 3 \\
\hline Rivina humilis L. CSJ1333, MDRS10 & $\mathrm{Hi}$ & $\mathrm{Au}$ & $\mathrm{Fl}$ & 1,8 \\
\hline \multicolumn{5}{|l|}{ Piperaceae } \\
\hline Peperomia angustata Kunth JAEJ601 & $\mathrm{Hi}$ & $\mathrm{Au}$ & $\mathrm{FI}$ & 1 \\
\hline
\end{tabular}


Apéndice 1. Continuación

\begin{tabular}{|c|c|c|c|c|}
\hline Familia/Especie/ Colector & FC & FN & FE & MC \\
\hline Peperomia asarifolia Schltdl. \& Cham. ARG \& GUR900 & $\mathrm{Hi}$ & $\mathrm{Au}$ & $\mathrm{Fl}$ & 9 \\
\hline Peperomia blanda (Jacq.) Kunth JAEJ489 & $\mathrm{Hi}$ & $\mathrm{Ru}$ & $\mathrm{Fl}$ & 10 \\
\hline Peperomia deppeana Schltdl. \& Cham. GUR40, 41 & $\mathrm{Hi}$ & Ep & $\mathrm{Fl}$ & 2 \\
\hline Peperomia galioides Kunth GUR, EMS \& AM40, 41, 41-bis, JAEJ174, 599 & $\mathrm{Hi}$ & $\mathrm{Au}$ & $\mathrm{Fl}$ & $1,2,7$ \\
\hline Peperomia heterodoxa Standl. \& Steyerm. DEB37176, 51973 Chis, G, Oax & $\mathrm{Hi}$ & Ep & $\mathrm{Fl}$ & 8,9 \\
\hline Peperomia hirta C. DC. JAEJ416, 457 & $\mathrm{Hi}$ & Ep & $\mathrm{Fl}$ & 8,10 \\
\hline Peperomia humilis A. Dietr. DEB52178 & $\mathrm{Hi}$ & Ep & $\mathrm{Fl}$ & 8 \\
\hline $\begin{array}{l}\text { Peperomia lanceolato-peltata C. DC. DEB70262, DEB \&ARS21566, } \\
\text { DEB \& BB55477, DEB \& FA48468, JAEJ243, 360, } 396\end{array}$ & $\mathrm{Hi}$ & $\mathrm{Ru}$ & $\mathrm{Fl}, \mathrm{Fr}$ & $7,8,9,11,12$ \\
\hline Peperomia macrandra C. DC. JAEJ425 & $\mathrm{Hi}$ & $\mathrm{Ru}$ & $\mathrm{Fl}$ & 8 \\
\hline Peperomia peltata C. DC. OFS1066 & $\mathrm{Hi}$ & $\mathrm{Ru}$ & $\mathrm{Fl}$ & 1 \\
\hline Peperomia quadrifolia (L.) Kunth. DEB37175, MCHB, MK, RD \& MNAs/n & $\mathrm{Hi}$ & Ep & $\mathrm{Fl}$ & 1,9 \\
\hline Peperomia succulenta C. DC. JAEJ389, 586 & $\mathrm{Hi}$ & Ep & $\mathrm{FI}$ & 1,11 \\
\hline Peperomia tenella (Sw.) A. Dietr. JAEJ669 & $\mathrm{Hi}$ & $\mathrm{Au}$ & $\mathrm{Fl}$ & 2 \\
\hline $\begin{array}{l}\text { Peperomia tetraphylla (G. Forst.) Hook. \& Arn. DEB \& ARS21583, } \\
\text { JAEJ388, OFS \& MAPF998 }\end{array}$ & $\mathrm{Hi}$ & Ep & $\mathrm{Fl}$ & 1,11 \\
\hline Peperomia tuerckheimii C. DC. DEB, ER \& PH13389 Chis, C & $\mathrm{Hi}$ & Ep & $\mathrm{Fl}$ & 10 \\
\hline Piper aduncum L. JAEJ766 & $\mathrm{Hi}$ & $\mathrm{Au}$ & $\mathrm{Fl}$ & 4 \\
\hline Piper amalago L. HETR32, JAEJ79, 145, 246, 658, 739, 754, JAEJ \& ALP220 & $\mathrm{Hi}$ & $\mathrm{Au}, \mathrm{Ru}$ & $\mathrm{Fl}, \mathrm{Fr}$ & $1,2,5,6,7,9$ \\
\hline Piper hispidum Sw. JAEJ755, MAPF833 & $\mathrm{Hi}$ & $\mathrm{Au}$ & $\mathrm{FI}$ & 1,11 \\
\hline Piper marginatum Jacq. JAEJ358 & $\mathrm{Hi}$ & $\mathrm{Ru}$ & $\mathrm{FI}$ & 6 \\
\hline Piper umbellatum L. CSJ; et al.13330 & $\mathrm{Hi}$ & $\mathrm{Au}$ & $\mathrm{FI}$ & 10 \\
\hline \multicolumn{5}{|l|}{ Polemoniaceae } \\
\hline Loeselia glandulosa (Cav.) G. Don. JAEJ575 & $\mathrm{Hi}$ & $\mathrm{Au}$ & $\mathrm{FI}$ & 1 \\
\hline \multicolumn{5}{|l|}{ Polygalaceae } \\
\hline Polygala chiapensis S.F. Blake. DEB37206, OFC234 Chis & $\mathrm{Hi}$ & $\mathrm{Au}$ & $\mathrm{FI}$ & 8,9 \\
\hline Polygala consobrina S.F. Blake. DEB37242 & $\mathrm{Hi}$ & $\mathrm{Au}$ & $\mathrm{Fl}$ & 9 \\
\hline Polygala costaricensis Chodat. JAEJ434, LFMJ4 & $\mathrm{Hi}$ & $\mathrm{Au}$ & $\mathrm{Fr}$ & 8,10 \\
\hline $\begin{array}{l}\text { Polygala floribunda Benth. AM, GUR \& EMS97, DEB13997, 51977, 9033, } \\
\text { FMG107, GD29723, GD, MSS, OT, EMS \& JDs/n, GUR7, JAEJ471, } 718\end{array}$ & $\mathrm{Hi}$ & $\mathrm{Au}$ & $\mathrm{FI}$ & $\begin{array}{c}1,2,3,8 \\
10,11\end{array}$ \\
\hline Polygala microtricha S.F. Blake. DEB51997, DEB52165 & - & - & - & 8 \\
\hline Polygala monticola var. brizoides (A. St.-Hil.) Steyerm. DEB52164 & $\mathrm{Hi}$ & $\mathrm{Au}$ & $\mathrm{Fl}$ & 8 \\
\hline Polygala rivinifolia Kunth. DEB37172, DEB, ER \& PH13543 & $\mathrm{Hi}$ & $\mathrm{Au}$ & $\mathrm{Fl}$ & 9,10 \\
\hline Securidaca diversifolia (L) Blake DEB \& EMC34027, MAMA1122, MAMAs/n & $\mathrm{Li}$ & $\mathrm{Au}$ & $\mathrm{Fl}$ & 1,3 \\
\hline \multicolumn{5}{|l|}{ Polygonaceae } \\
\hline Antigonon guatimalense Meisn. AML15, RML904 & $\mathrm{Li}$ & $\mathrm{Au}$ & - & 5,9 \\
\hline $\begin{array}{l}\text { Antigonon leptopus Hook \& Arn. AM, GUR \& EMS63, AML18, ARG958, } \\
\text { DEB20024, DLA35, GKGR21 }\end{array}$ & $\mathrm{Li}$ & $\mathrm{Au}$ & $\mathrm{Fl}$ & $2,5,9,10$ \\
\hline Coccoloba acapulcensis Standl. HTR14 & Á & $\mathrm{Au}$ & - & 4 \\
\hline Coccoloba barbadensis Jacq.GUR18, JAEJ110 & Á, $\mathrm{Ab}$ & $\mathrm{Au}$ & $\mathrm{Fl}, \mathrm{Fr}$ & 2,6 \\
\hline Coccoloba cozumelensis Hemsl. GUR, EMS \& AM18 & Á & $\mathrm{Au}$ & $\mathrm{Fr}$ & 2 \\
\hline Coccoloba diversifolia Jacq. DEB51953, JAEJ176 & Á & $\mathrm{Au}$ & $\mathrm{Fr}$ & 7,8 \\
\hline Coccoloba hondurensis Lundell. DEB30033, 37230, GUR, EMS \& AM18 & Á & $\mathrm{Au}$ & $\mathrm{Fl}$ & $1,9,12$ \\
\hline Coccoloba montana Standl. DEB51583 & Á & $\mathrm{Au}$ & - & 7 \\
\hline $\begin{array}{l}\text { Gymnopodium antigonoides (B.L. Rob. ex Millsp. \& Loes.) S.F. Blake. } \\
\text { AM, GUR \& EMS18-BIS, DN5518B, JAEJ92 }\end{array}$ & $\mathrm{Ab}$ & $\mathrm{Au}$ & $\mathrm{Fl}$ & $2,3,6$ \\
\hline Podopterus mexicanus Bonpl. WCH3455 & - & $\mathrm{Au}$ & - & 5 \\
\hline Polygonum hispidum Kunth DEB9195 & $\mathrm{Hi}$ & $\mathrm{Au}$ & - & 2 \\
\hline \multicolumn{5}{|l|}{ Portulacaceae } \\
\hline Talinum paniculatum (Jacq.) Gaertn. JAEJ147 & $\mathrm{Hi}$ & $\mathrm{Au}$ & $\mathrm{Fr}$ & 7 \\
\hline
\end{tabular}


Apéndice 1. Continuación

\begin{tabular}{|c|c|c|c|c|}
\hline Familia/Especie/ Colector & FC & FN & $\mathrm{FE}$ & MC \\
\hline \multicolumn{5}{|l|}{ Ranunculaceae } \\
\hline Clematis dioica L. AM, GUR \& EMS83, JAEJ452, LFMJ26 & $\mathrm{Li}$ & $\mathrm{Au}$ & $\mathrm{Fl}$ & 2,10 \\
\hline \multicolumn{5}{|l|}{ Rhamnaceae } \\
\hline Ceanothus coeruleus Lag. JMHJr & - & - & - & - \\
\hline Colubrina arborescens (Mill.) Sarg. & Á & $\mathrm{Au}$ & $\mathrm{Fl}$ & 6 \\
\hline Colubrina elliptica (Sw.) Brizicky \& W.L. Stern DEB \& FSKs/n, DEB \& FSKs/n & Á & $\mathrm{Au}$ & - & 7,10 \\
\hline Gouania eurycarpa Standl. CSJ; et al.13334 & $\mathrm{Li}$ & $\mathrm{Au}$ & - & 10 \\
\hline Gouania lupuloides (L.) Urb. AM, GUR \& EMS84 & $\mathrm{Li}$ & $\mathrm{Au}$ & - & 2 \\
\hline Gouania polygama (Jacq.) Urb. JAEJ473 & $\mathrm{Li}$ & $\mathrm{Au}$ & $\mathrm{Fl}$ & 10 \\
\hline Sageretia elegans (H.B.K.) Brongn VMSL1113 & $\mathrm{Ab}$ & $\mathrm{Au}$ & - & 1 \\
\hline \multicolumn{5}{|l|}{ Rosaceae } \\
\hline Acaena elongata L. JMHJr32 & $\mathrm{Ab}$ & $\mathrm{Au}$ & 6 & 9 \\
\hline Eriobotrya japonica (Thunb.) Lindl. JAEJ779 & $\mathrm{Ab}$ & $\mathrm{Au}$ & $\mathrm{FI}$ & 6 \\
\hline Holodiscus argenteus (L. f.) Maxim. DEB9305 & $\mathrm{Ab}$ & $\mathrm{Au}$ & - & 3 \\
\hline Holodiscus fissus (Lindl.) C.K. Schneid. JMHJrs/n & $\mathrm{Ab}$ & $\mathrm{Au}$ & - & 9 \\
\hline Rubus trilobus Moc. \& Sessé ex Ser. JMHJrs/n & $\mathrm{Ab}$ & $\mathrm{Au}$ & - & 8 \\
\hline \multicolumn{5}{|l|}{ Rubiaceae } \\
\hline Alibertia edulis (Rich.) A. Rich. ex DC. GUR138 & Á & $\mathrm{Au}$ & $\mathrm{Fr}$ & 10 \\
\hline Borreria laevis (Lam.) Griseb. JM7, LFMJ19 & $\mathrm{Hi}$ & $\mathrm{Au}$ & $\mathrm{Fl}$ & 10,11 \\
\hline Borreria ocymoides fo. tenuis Chod. \& Hassler. ARG941, RTC, EC \& MHs/n & $\mathrm{Hi}$ & $\mathrm{Au}$ & $\mathrm{FI}$ & 9,10 \\
\hline Bouvardia leiantha Benth. DEB28168, JAEJ \& ALP486 & $\mathrm{Hi}$ & $\mathrm{Au}$ & $\mathrm{Fl}$ & 9,10 \\
\hline Bouvardia longiflora (Cav.) Kunth. GU \& EMS \& AMG45, RCC26, SRD48 & $\mathrm{Ab}, \mathrm{Hi}$ & $\mathrm{Au}$ & $\mathrm{Fl}$ & 2,5 \\
\hline Bouvardia viminalis Schltdl. JM16 & $\mathrm{Hi}$ & $\mathrm{Au}$ & $\mathrm{FI}$ & 11 \\
\hline Calycophyllum candidissimum (Vahl) DC. JAEJ330, 567 & Á & $\mathrm{Au}$ & $\mathrm{Fl}$ & 9,12 \\
\hline $\begin{array}{l}\text { Chiococca alba (L.) Hitchc. CSJ13246, EUVP20, GUR, EMS \& AM8, } \\
\text { JAEJ374, 505, JAEJ \& ALP274 }\end{array}$ & Á, $A b$ & $\mathrm{Au}$ & $\mathrm{Fl}, \mathrm{Fr}$ & $2,8,10,11$ \\
\hline Coffea arabica L. JAEJ239 & Á & $\mathrm{Au}$ & $\mathrm{Fr}$ & 7 \\
\hline Coutarea hexandra (Jacq.) K. Schum. JAEJ773 & Á & $\mathrm{Au}$ & $\mathrm{FI}$ & 6 \\
\hline Crusea calocephala DC. JAEJ439 & Á & $\mathrm{Au}$ & $\mathrm{Fl}$ & 10 \\
\hline Crusea hispida var. hispida RTC, EC \& MHs/n & $\mathrm{Hi}$ & $\mathrm{Au}$ & - & 10 \\
\hline Crusea longiflora (Willd. ex Roem. \& Schult.) W.R. Anderson. CSJ13319 & $\mathrm{Hi}$ & $\mathrm{Au}$ & - & 10 \\
\hline Crusea psyllioides (Kunth) W.R. Anderson. DEB, PRH \& ER13491 & $\mathrm{Hi}$ & $\mathrm{Au}$ & - & 10 \\
\hline Deppea anisophylla L.O. Williams JAEJ221 & $\mathrm{Hi}$ & $\mathrm{Ru}$ & $\mathrm{FI}$ & 7 \\
\hline Exostema caribaeum (Jacq.) Roem. \& Schult. JAEJ \& JMM201 & Á & $\mathrm{Ru}$ & $\mathrm{FI}$ & 7 \\
\hline Guettarda elliptica Sw. JAEJ \& ALP300 & $\mathrm{Ab}$ & $\mathrm{Au}$ & $\mathrm{Fr}$ & 8 \\
\hline $\begin{array}{l}\text { Guettarda subcapitata C.M. Taylor DEB13388, 27138, DEB } \\
\quad \text { \& ARS 21594, OT \& JLVR6689 Chis, C }\end{array}$ & Á, $A b$ & $\mathrm{Au}$ & $\mathrm{FI}$ & $4,8,10,11$ \\
\hline $\begin{array}{l}\text { Hamelia patens Jacq. DCLD, DEB \& FSKs/n, DNN, ELLs/n, ERB13, } \\
\text { JJVJ18, RMKs/n, SGT37 }\end{array}$ & $\mathrm{Ab}$ & $\mathrm{Au}$ & $\mathrm{Fl}, \mathrm{Fr}$ & $5,6,7,8,9$ \\
\hline Hamelia patens var. glabra Jacq. JAEJ391 & $\mathrm{Ab}$ & $\mathrm{Au}$ & $\mathrm{Fr}$ & 8 \\
\hline Hamelia patens var. patens Jacq. JAEJ137 & $\mathrm{Ab}$ & $\mathrm{Au}$ & $\mathrm{Fl}, \mathrm{Fr}$ & 7 \\
\hline Hillia parasitica Jacq. FMG7665 & $\mathrm{Li}$ & Ep & $\mathrm{Fl}$ & 1 \\
\hline Margaritopsis microdon (DC.) C.M. Taylor. JAEJ238, JAEJ \& ALP304 & Á, $A b$ & $\mathrm{Au}$ & $\mathrm{Fl}$ & 7,8 \\
\hline Psychotria chagrensis Standl. GD29715 & $\mathrm{Ab}$ & $\mathrm{Au}$ & - & 11 \\
\hline $\begin{array}{l}\text { Psychotria erythrocarpa Schltdl. DDO24, DEB \& ARSs/n, DEB \& FSKs/n, } \\
\text { EUVP6, JAEJ84, 115, 179, 327, 393, JAEJ Y ALP310, VMSL1110 }\end{array}$ & Á, $A b$ & $\mathrm{Au}$ & $\mathrm{Fl}, \mathrm{Fr}$ & $\begin{array}{l}1,6,7,8 \\
9,10,11\end{array}$ \\
\hline Psychotria horizontalis Sw. JAEJ164 & $\mathrm{Hi}$ & $\mathrm{Au}$ & $\mathrm{FI}$ & 7 \\
\hline Psychotria mirandae C.W. Ham. JAEJ237 Chis, Ver & Á & $\mathrm{Au}$ & $\mathrm{FI}$ & 7 \\
\hline Psychotria pubescens Sw. JAEJ \& ALP279, JAEJ \& JMM197 & Á & $\mathrm{Au}, \mathrm{Ru}$ & $\mathrm{Fl}$ & 7,8 \\
\hline Randia aculeata L. CMPs/n, GUR, EMS \& AM25, MPMMs/n, MVMs/n, & $\mathrm{Ab}$ & $\mathrm{Au}$ & $\mathrm{Fr}$ & 1,11 \\
\hline
\end{tabular}


Apéndice 1. Continuación

\section{Familia/Especie/ Colector}

$\mathrm{PMMs} / \mathrm{n}, \mathrm{VMM} \& \mathrm{IVSHO3}$

Randia cinerea (Fernald) Standl. DEB39959, JAEJ208

Randia guatemalensis Standl. MAMAs/n

Randia laetevirens Standl. GUR45, GUR \& EMS \& AMG25, JAEJ \& ALP244

Randia monantha Benth. JAEJ, 561

Rondeletia cordata Benth. JAEJ654, 738

Spermacoce remota Lam. JAEJ \& JMM272

\section{Rutaceae}

Amyris belizensis Lundell. AM, GUR \& EMS35, GUR35

Amyris elemifera L. FMG7755, 7904

Decatropis bicolor (Zucc.) Radlk. DEB \& FSKs/n

Decatropis paucijuga (Donn. Sm.) Loes. DDR 379, DEB379, 9609, 10672, 14694, 30021

Zanthoxylum aguilarii Standl. \& Steyerm. EMS, OT, MSS, GD \& JD8577, FMG7667, GUR5

Zanthoxylum culantrillo Kunth. CSJ; et al.13256, DEB \& RFT30029

Zanthoxylum procerum Donn Smith. YNG34

Zanthoxylum riedelianum Engl. JAEJ119

Salicaceae

Salix humboldtiana Willd. JAEJ727

Sapindaceae

Cupania dentata DC. ARP104, JAEJ421

Dodonaea aff. viscosa Jacq. JAEJ637

Dodonaea viscosa Jacq. GUR, EMS \& AM4, JAEJ634

Exothea paniculata (Juss.) Radlk. JAEJ717

Paullinia costaricensis Radlk. AM, GUR \& EMS9, JAEJ712

Paullinia fuscescens Kunth EMSs/n, GUR, EMS \& AM62, MAMA,

TBC \& DPHs/n

Sapindus saponaria L. DEB \&EMCs/n, JAEJ728

Serjania acuta Triana \& Planch. DEB \& FSKs/n

Serjania cardiospermoides Schltdl. \& Cham. AM, GUR \& EMS104, ARG,

RH \& LH1744, JAEJ \& ELM383

Serjania goniocarpa Radlk. AM, GUR \& EMS79

Serjania grosii Schltdl. AM, GUR \& EMS104

Serjania lobulata Standl. \& Steyerm. DEB \& FSKs/n

Serjania phaseoloides Standl. \& Steyerm. ARG897

Serjania triquetra Radlk. JAEJ \& ALP266, 309, MDRS7

Talisia floresii Standl. JAEJ716

Thouinia acuminata S. Watson DEB \& FA48451, DEB \& FSK28170

\section{Sapotaceae}

Bumelia laetevirens Hemsl. GU \& EMS \& AMG27

Chrysophyllum mexicanum Brandegee ex Standl. DEB9032, GUR, EMS

\& AM15, JAEJ144, 584, OFS1057, RMC638

Chrysophyllum oliviforme L. DEB7490

Pouteria sapota (Jacq.) H.E. Moore \& Stearn JAEJ701

Sideroxylon capiri (A. DC.) Pittier AM1 A

Sideroxylon capiri subsp. tempisque (Pittier) T.D. Penn. AM, GUR \& EMS1

Sideroxylon laetevirens (Baill.) Engl. DNs/n

Sideroxylon obtusifolium (Humb. ex Roem. \& Schult.) T.D. Penn.

ARG \& GUR910, MMO6

FC FN $\quad$ FE $\quad$ MC

Á

$\mathrm{Ab}$

$\mathrm{Ab}$

Á

$\mathrm{Ab}$

$\mathrm{Hi}$

$\mathrm{Ab}$

$\mathrm{Ab}$

Á

$\mathrm{Ab}$

Á, Ab

Á

Á

Á

Á

Á

Á

Á, $\mathrm{Li}$

Á

$\mathrm{Li}$

$\mathrm{Li}$

Á

$\mathrm{Li}$

Li

Li

$\mathrm{Li}$

$\mathrm{Li}$

$\mathrm{Li}$

$\mathrm{Li}$

Á

Á

Á

Á

$\mathrm{Ab}$

Á

Á

Á

Á

Á
Au

$\mathrm{Au}$

$\mathrm{Au}$

$\mathrm{Au}$

$\mathrm{Au}, \mathrm{Ru}$

$\mathrm{Au}$

$\mathrm{Au}$

$\mathrm{Au}$

$\mathrm{Au}$

$\mathrm{Au}$

$\mathrm{Au}$

Au

$\mathrm{Au}$

$\mathrm{Au}$

$\mathrm{Au}$

Au

$\mathrm{Au}$

$\mathrm{Au}$

$\mathrm{Au}$

$\mathrm{Au}$

$\mathrm{Au}$

$\mathrm{Au}$

$\mathrm{Au}$

$\mathrm{Au}$

Au

$\mathrm{Au}$

$\mathrm{Au}$

$\mathrm{Au}$

$\mathrm{Au}$

Au

$\mathrm{Au}$

Au

$\mathrm{Au}$

$\mathrm{Au}$

$\mathrm{Au}$

$\mathrm{Au}$

$\mathrm{Au}$

$\mathrm{Au}$

$\mathrm{Au}$

$\begin{array}{cc}\mathrm{Fl} & 7,9 \\ - & 3 \\ \mathrm{Fr} & 2,7 \\ \mathrm{Fl}, \mathrm{Fr} & 12 \\ \mathrm{Fl} & 2,4 \\ \mathrm{Fl} & 8\end{array}$

- 2

- 1

- 7

$\begin{array}{ll}- & 11\end{array}$

Fr $\quad 1,2$

$\begin{array}{ll}- & 10\end{array}$

- 1

FI 6

Fl 4

$\mathrm{Fl}, \mathrm{Fr} \quad 1,8$

$\mathrm{Fr} 2$

$\mathrm{Fr} \quad 2$

Fl 3

$\mathrm{Fr} \quad 2,3$

Fr $1,2,3,11$

Fr $\quad 1,4$

- 2

$\mathrm{Fr}, \mathrm{Fl} \quad 2,7,10$

$\begin{array}{ll}- & 2\end{array}$

- 2

$\begin{array}{ll}- & 10\end{array}$

Fr $\quad 9$

$\mathrm{Fl}, \mathrm{Fr} \quad 8$

$\mathrm{FI} \quad 3$

Fl $\quad 9,12$

- 2

$\mathrm{Fl}, \mathrm{Fr} \quad 1,2,7,9$

- 3

E 3

- 2

- 2

- 3

$\mathrm{Fr} \quad 2,11$ 
Apéndice 1. Continuación

\begin{tabular}{|c|c|c|c|c|}
\hline Familia/Especie/ Colector & FC & FN & FE & MC \\
\hline Sideroxylon persimile (Hemsl.) T.D. Penn. EC \&HCs/n & Á & $\mathrm{Au}$ & - & 12 \\
\hline Sideroxylon persimile spp. persimile DEBs/n & Á & $\mathrm{Au}$ & - & 5 \\
\hline Sideroxylon salicifolium (L.) Lam. DEBs/n, DEB27275 & Á & $\mathrm{Au}$ & - & 1,8 \\
\hline \multicolumn{5}{|l|}{ Scrophulariaceae } \\
\hline Capraria biflora L. JAEJ151 & $\mathrm{Ab}$ & $\mathrm{Au}$ & - & 7 \\
\hline Castilleja arvensis Schltdl. \& Cham. GCCs/n, JAEJ539 & $\mathrm{Hi}$ & $\mathrm{Au}$ & $\mathrm{Fl}$ & 2,12 \\
\hline Escobedia guatemalensis Loes. DEB \& FSKs/n Chis, G & $\mathrm{Hi}$ & $\mathrm{Au}$ & - & 8 \\
\hline Lamourouxia viscosa Kunth GCCs/n, JAEJ488, 573, PAF \& EJLTTs/n & $\mathrm{Hi}$ & $\mathrm{Au}$ & $\mathrm{Fl}$ & $1,2,10,11$ \\
\hline Melasma physalodes (D. Don) Melch. DEB \& FSKs/n & $\mathrm{Hi}$ & $\mathrm{Au}$ & - & 10 \\
\hline Russelia multiflora Sims. JAEJ242 & $\mathrm{Hi}$ & $\mathrm{Au}$ & $\mathrm{Fl}$ & 7 \\
\hline Russelia purpusii Brandegee JAEJ328 & $\mathrm{Li}$ & $\mathrm{Au}$ & $\mathrm{Fl}$ & 9 \\
\hline Russelia sarmentosa Jacq. ADS971 & $\mathrm{Hi}$ & $\mathrm{Au}$ & $\mathrm{Fl}$ & 3 \\
\hline \multicolumn{5}{|l|}{ Simaroubaceae } \\
\hline $\begin{array}{l}\text { Alvaradoa amorphoides Liebm AGP74, DEB, PRH \& Ers/n, } \\
\text { FDAL25, JAEJ521, } 556\end{array}$ & Á & $\mathrm{Au}$ & $\mathrm{Fl}$ & $2,10,11,12$ \\
\hline Picramnia antidesma Sw. GKGR26 & Á & $\mathrm{Au}$ & $\mathrm{Fr}$ & 5 \\
\hline Picramnia matudae Lundell GKGR27 Chis & Á & $\mathrm{Au}$ & $\mathrm{Fr}$ & 5 \\
\hline \multicolumn{5}{|l|}{ Solanaceae } \\
\hline Capsicum ciliatum (Kunth) Kuntze DEB51520 & $\mathrm{Ab}$ & $\mathrm{Au}$ & - & 7 \\
\hline Capsicum frutescens L. JAEJ143 & $\mathrm{Ab}$ & $\mathrm{Au}$ & $\mathrm{Fl}$ & 7 \\
\hline Cestrum diurnum L. RML913 & $\mathrm{Ab}$ & $\mathrm{Au}$ & - & 5 \\
\hline Cestrum guatemalense Francey JAEJ \& ALP281 Chis, C & $\mathrm{Ab}$ & $\mathrm{Au}$ & $\mathrm{Fr}$ & 8 \\
\hline Cestrum nocturnum L. DEB52204 & $\mathrm{Ab}$ & $\mathrm{Au}$ & - & 8 \\
\hline Cyphomandra betacea (Cav.) Sendtn. GUR156 & Á & $\mathrm{Au}$ & $\mathrm{Fr}$ & 3 \\
\hline Datura inoxia Mill. DEB \& EMC34038 & $\mathrm{Hi}$ & $\mathrm{Au}$ & - & 3 \\
\hline Juanulloa mexicana (Schltdl.) Miers FMG7520 & $\mathrm{Ab}$ & $\mathrm{Pa}$ & - & 1 \\
\hline Lycianthes armentalis J.L. Gentry EGA47, HETR31, JJVJ26 & $\mathrm{Ab}, \mathrm{Hi}$ & $\mathrm{Au}$ & - & 5,6 \\
\hline Lycianthes ciliolata (M. Martens \& Galeotti) Bitter DEB10644 & $\mathrm{Hi}$ & $\mathrm{Au}$ & - & 7 \\
\hline Lycianthes lenta (Cav.) BitAur JAEJ139, JAEJ \& ALP273, PAF \& EJLTTs/n & $\mathrm{Ab}$ & $\mathrm{Au}$ & $\mathrm{Fr}$ & $7,8,11$ \\
\hline Lycianthes stephanocalyx (Brandegee) Bitter DEB40004 & $\mathrm{Ab}$ & $\mathrm{Au}$ & - & 9 \\
\hline Markea sp.1 DEB9037 & $\mathrm{Li}$ & Ep & - & 2 \\
\hline Physalis gracilis Miers. ARG898, DEB39978, 39990 & $\mathrm{Hi}$ & $\mathrm{Au}$ & - & 9 \\
\hline Physalis maxima Mill. JAEJ \& ALP306 & $\mathrm{Hi}$ & $\mathrm{Au}$ & $\mathrm{Fl}$ & 8 \\
\hline Solandra nizandensis Matuda JAEJ225 & $\mathrm{Ab}$ & $\mathrm{Ru}$ & $\mathrm{Fl}$ & 7 \\
\hline Solanum chrysotrichum Schltdl. JAEJ470 & $\mathrm{Ab}$ & $\mathrm{Au}$ & $\mathrm{Fl}$ & 10 \\
\hline Solanum erianthum D. Don CHP3252, JAEJ106 & $\mathrm{Hi}$ & $\mathrm{Au}$ & $\mathrm{Fr}$ & 3,6 \\
\hline Solanum glaucescens Zucc. DEB26219 & $\mathrm{Li}$ & $\mathrm{Au}$ & - & 7 \\
\hline Solanum lanceifolium Jacq. DEB51967, 37160 & $\mathrm{Li}$ & $\mathrm{Au}$ & - & 8 \\
\hline $\begin{array}{l}\text { Solanum lanceolatum Cav. DEB13914, DRG18, EMS8597, GUR, } \\
\text { EMS \& AM13, JMHJrs/n }\end{array}$ & $\mathrm{Ab}$ & $\mathrm{Au}$ & $\mathrm{Fl}, \mathrm{Fr}$ & $1,5,9,10,11$ \\
\hline Solanum lepidotum Dunal JAEJ186 & $\mathrm{Ab}$ & $\mathrm{Au}$ & $\mathrm{Fl}$ & 7 \\
\hline Solanum nudum Dunal DEB51992, 52159 & $\mathrm{Ab}$ & $\mathrm{Au}$ & - & 8 \\
\hline Solanum ochraceo-ferrugineum (Dunal) Fernald. RCC20 & $\mathrm{Ab}$ & $\mathrm{Au}$ & $\mathrm{Fl}$ & 5 \\
\hline Solanum roei Ugent \& H.H. Iltis. DEB37189 & $\mathrm{Hi}$ & $\mathrm{Au}$ & - & 9 \\
\hline Solanum somniculentum Kuntze ex SchltdI.DEB10644 & - & - & - & 7 \\
\hline Solanum suaveolens Kunth \& C.D. Bouché. DEB39987 & $\mathrm{Li}$ & $\mathrm{Au}$ & - & 9 \\
\hline Solanum torvum Sw. AM6, AM, GUR \& EMS490, FDAL30, GUR13 & Á, $\mathrm{Ab}, \mathrm{Hi}$ & $\mathrm{Au}$ & $\mathrm{Fl}, \mathrm{Fr}$ & 2,10 \\
\hline Solanum umbellatum Mill. PAF \& EJLTTs/n & $\mathrm{Ab}$ & $\mathrm{Au}$ & - & 11 \\
\hline Solanum verbascifolium L. DEB9048 & Á & $\mathrm{Au}$ & - & 2 \\
\hline Solanum wendlandii Hook. F. DEB9613, DEB \& FSKs/n & $\mathrm{Li}$ & $\mathrm{Au}$ & - & 4,12 \\
\hline
\end{tabular}


Apéndice 1. Continuación

\begin{tabular}{|c|c|c|c|c|}
\hline Familia/Especie/ Colector & FC & FN & FE & MC \\
\hline Witheringia stramoniifolia Kunth DEB39989, 9042 & $\mathrm{Ab}$ & $\mathrm{Au}$ & - & 9 \\
\hline \multicolumn{5}{|l|}{ Sterculiaceae } \\
\hline Ayenia mexicana Turcz. DEB51986, DEB, ER \& PH13546, DEB, PRH \& Ers/n & $\mathrm{Ab}$ & $\mathrm{Au}$ & - & 8,10 \\
\hline Ayenia micrantha Standl. DEB13884, 26232, DEB \& FSKs/n & $\mathrm{Ab}$ & $\mathrm{Au}$ & - & 7,10 \\
\hline Byttneria catalpifolia Jacq. DEB39922, JAEJ97, 99 & $\mathrm{Li}$ & $\mathrm{Au}$ & $\mathrm{Fl}, \mathrm{Fr}$ & 6,9 \\
\hline Guazuma ulmifolia Lam.DTR31, ERB19, JAEJ87, 141 & Á, $\mathrm{Hi}$ & $\mathrm{Au}$ & $\mathrm{Fl}, \mathrm{Fr}$ & $5,6,7,10$ \\
\hline Helicteres baruensis Jacq. EUVP23 & $\mathrm{Ab}$ & $\mathrm{Au}$ & - & 10 \\
\hline Melochia nodiflora Sw. EMS, OT, MSS \& GDs/n & $\mathrm{Hi}$ & $\mathrm{Au}$ & - & 11 \\
\hline $\begin{array}{l}\text { Melochia nudiflora Standl. \& L.O. Williams. DEB13860, DEB \& ARSs/n, } \\
\text { DEB \& ARS21573, DEB \& FSKs/n }\end{array}$ & $\mathrm{Hi}$ & $\mathrm{Au}$ & - & 10,11 \\
\hline Melochia sp.1 DEB37249 & $\mathrm{Ab}$ & $\mathrm{Au}$ & $\mathrm{Fl}$ & 9 \\
\hline Waltheria indica L. DEB13532, RTC6362 & $\mathrm{Hi}$ & $\mathrm{Au}$ & - & 10 \\
\hline Waltheria rotundifolia Schrank DEB, ER \& PH13532 & $\mathrm{Ab}$ & $\mathrm{Au}$ & - & 10 \\
\hline \multicolumn{5}{|l|}{ Styracaceae } \\
\hline Styrax argenteus var. argenteus DEB33804, 39966 & Á & $\mathrm{Au}$ & - & 2,9 \\
\hline \multicolumn{5}{|l|}{ Theaceae } \\
\hline $\begin{array}{l}\text { Ternstroemia tepezapote Schltdl. \& Cham. DEB13999, GKGR29, JAEJ691, } \\
\quad \text { 480, 745, JAEJ \& ALP302 }\end{array}$ & Á & $\mathrm{Au}$ & $\mathrm{Fl}, \mathrm{Fr}$ & $3,4,5,8,10$ \\
\hline \multicolumn{5}{|l|}{ Theophrastaceae } \\
\hline $\begin{array}{l}\text { Jacquinia macrocarpa Cav. ARG526, EMS20142, EL6680, JAEJ100, } \\
\text { JTBJs/n, LLIs/n, MDRS8 }\end{array}$ & $\mathrm{Ab}$ & $\mathrm{Au}$ & $\mathrm{Fl}, \mathrm{Fr}$ & $4,5,6,8,9$ \\
\hline Jacquinia macrocarpa spp. macrocarpa LIDs/n & $\mathrm{Ab}$ & $\mathrm{Au}$ & - & 3 \\
\hline \multicolumn{5}{|l|}{ Thymelaeaceae } \\
\hline $\begin{array}{l}\text { Daphnopsis americana (Mill.) J.R. Johnst. DEB35065, 50642, DEB } \\
\quad \text { \& EMC23565, JAEJ \& ALP276 }\end{array}$ & Á & $\mathrm{Au}$ & $\mathrm{Fl}$ & $1,4,5,8$ \\
\hline \multicolumn{5}{|l|}{ Tiliaceae } \\
\hline Belotia grewiifolia A. Rich. ARP112 & Á & $\mathrm{Au}$ & - & 1 \\
\hline Corchorus hirtus L. DEB41494, 41504 & $\mathrm{Hi}$ & $\mathrm{Au}$ & - & 11 \\
\hline Corchorus siliquosus L. TBC \& DPHs/n & $\mathrm{Hi}$ & $\mathrm{Au}$ & - & 2 \\
\hline Heliocarpus americanum L. CRB357, JAEJ546, TBC \& DPHs/n & Á & $\mathrm{Au}$ & $\mathrm{Fr}$ & $2,11,12$ \\
\hline Heliocarpus appendiculatus Turcz. AM, GUR \& EMS77 & Á & $\mathrm{Au}$ & - & 2 \\
\hline Heliocarpus mexicanus (Turcz.) Sprague. TBC \& DPHs/n & Á & $\mathrm{Au}$ & - & 2 \\
\hline Heliocarpus terebinthinaceus (DC.) Hochr. DEB41502 & Á & $\mathrm{Au}$ & - & 11 \\
\hline Luehea speciosa Willd. DEB41533 & Á & $\mathrm{Au}$ & - & 11 \\
\hline $\begin{array}{l}\text { Triumfetta speciosa Seem. DEB14002, DEB \& ARSs/n, DEB \& ARS21540, } \\
\text { DEB \& FSKs/n, GD29754, GD, MSS, OT, EMS \& JDs/n, JAEJ466 }\end{array}$ & $\mathrm{HI}$ & $\mathrm{Au}$ & $\mathrm{Fl}$ & 10,11 \\
\hline \multicolumn{5}{|l|}{ Ulmaceae } \\
\hline Celtis caudata Planch. DEB \& RFT30026 & Á & $\mathrm{Au}$ & - & 12 \\
\hline Celtis iguanaea (Jacq.) Sarg. JAEJ180 & $\mathrm{Li}$ & $\mathrm{Au}$ & $\mathrm{Fr}$ & 7 \\
\hline Trema micrantha (L.) Blume EPEs/n, JAEJ187 & Á & $\mathrm{Au}$ & $\mathrm{Fl}$ & 2,7 \\
\hline \multicolumn{5}{|l|}{ Urticaceae } \\
\hline Boehmeria radiata W.C. Burger JAEJ740 & $\mathrm{Ab}$ & $\mathrm{Ru}$ & $\mathrm{Fl}$ & 4 \\
\hline Hemistylus odontophylla Wedd. CSJ13321 & $\mathrm{Ab}$ & $\mathrm{Au}$ & - & 10 \\
\hline Myriocarpa longipes Liebm. JAEJ315, 318, 579 & Á, $A b$ & $\mathrm{Au}$ & $\mathrm{Fl}, \mathrm{Fr}$ & $1,9,10$ \\
\hline Pilea glabra S. Watson JAEJ769 & $\mathrm{Ab}$ & $\mathrm{Au}$ & E & 8 \\
\hline Pilea macrophylla Rusby JAEJ382 & $\mathrm{Hi}$ & $\mathrm{Ru}$ & $\mathrm{Fl}$ & 1 \\
\hline \multicolumn{5}{|l|}{ Valerianaceae } \\
\hline Valeriana candolleana Gardner. JAEJ240 & $\mathrm{Hi}$ & $\mathrm{Ru}$ & $\mathrm{FI}$ & 7 \\
\hline Valeriana cucurbitifolia Standl. DEB10657, 14410, 27163 Chis, G, Oax & $\mathrm{Hi}$ & $\mathrm{Au}$ & $\mathrm{Fl}$ & 7,8 \\
\hline Valeriana palmeri A. Gray. DEB13897, 52166, DEB, ER \& PH13508 & $\mathrm{Hi}$ & $\mathrm{Au}$ & $\mathrm{Fl}$ & 8,10 \\
\hline
\end{tabular}


Apéndice 1. Continuación

\begin{tabular}{|c|c|c|c|c|}
\hline Familia/Especie/ Colector & FC & FN & FE & MC \\
\hline \multicolumn{5}{|l|}{ Verbenaceae } \\
\hline Bouchea prismatica (L.) Kuntze. DEB70051, JAEJ166 & $\mathrm{Hi}$ & $\mathrm{Au}$ & $\mathrm{Fl}$ & 7,9 \\
\hline Citharexylum mocinnii D. Don. MH \& AL8616 & Á & $\mathrm{Au}$ & - & 11 \\
\hline Duranta guatemalensis Moldenke. DEB51989 Chis, G & $\mathrm{Ab}$ & $\mathrm{Au}$ & - & 8 \\
\hline $\begin{array}{l}\text { Lantana achyranthifolia Desf. DEB52201, DEB, ER \& PH13526, DEB, } \\
\text { PRH \& Ers/n, JAEJ163 }\end{array}$ & $\mathrm{Ab}, \mathrm{Hi}$ & $\mathrm{Au}$ & $\mathrm{Fl}$ & $7,8,10$ \\
\hline Lantana camara L. DEB10653, DEB, ER \& PH13505 & $\mathrm{Hi}$ & $\mathrm{Au}$ & $\mathrm{Fl}$ & 7,10 \\
\hline Lantana costaricensis Hayek. DEBs/n & $\mathrm{Hi}$ & $\mathrm{Au}$ & - & 10 \\
\hline $\begin{array}{l}\text { Lantana urticifolia Mill. AFM \& ARMs/n, DEB13916, DEB, PRH \& Ers/n, } \\
\text { JAEJ114, OFS1055, VMSL1111 }\end{array}$ & $\mathrm{Ab}, \mathrm{Hi}$ & $\mathrm{Au}$ & $\mathrm{Fl}$ & $1,6,7,8,10$ \\
\hline Lippia alba (Mill.) N.E. Br. RCC22 & $\mathrm{Hi}$ & $\mathrm{Au}$ & $\mathrm{Fl}$ & 5 \\
\hline Lippia cardiostegia Benth. DEB13921, 27267, 52002, 52158 & $\mathrm{Hi}$ & $\mathrm{Au}$ & $\mathrm{Fl}$ & 8,10 \\
\hline Lippia controversa Moldenke. JAEJ555 & $\mathrm{Hi}$ & $\mathrm{Au}$ & $\mathrm{Fl}$ & 12 \\
\hline Lippia myriocephala Schltdl. \& Cham. JMM \& JP \& RO \& MMM1742 & $\mathrm{Ab}$ & $\mathrm{Au}$ & $\mathrm{Fl}$ & 2 \\
\hline $\begin{array}{l}\text { Petrea volubilis L. AM, GUR \& EMS12, DEBs/n, EMS, ARG \& Ams/n, } \\
\text { HETR28, JAEJ729, JLNs/n. }\end{array}$ & $\mathrm{Li}$ & $\mathrm{Au}$ & $\mathrm{Fl}$ & $2,4,5$ \\
\hline Priva aspera Kunth DEB52157 & $\mathrm{Hi}$ & $\mathrm{Au}$ & - & 8 \\
\hline $\begin{array}{l}\text { Priva lappulacea (L.) Pers. ARGs/n, DEB37252, JAEJ \& JMM199, } \\
\text { RTC, EC \& MHs/n }\end{array}$ & $\mathrm{Hi}$ & $\mathrm{Au}, \mathrm{Ru}$ & $\mathrm{Fl}$ & $7,9,10$ \\
\hline Stachytarpheta cayennensis (Rich.) Vahl. DEB52003 & $\mathrm{Hi}$ & $\mathrm{Au}$ & $\mathrm{FI}$ & 8 \\
\hline Stachytarpheta frantzii Pol. AFM \& ARMs/n, DEB10660, 52142 & $\mathrm{Ab}$ & $\mathrm{Au}$ & $\mathrm{FI}$ & 7,8 \\
\hline Stachytarpheta mutabilis (Jacq.) Vahl JAEJ341 & $\mathrm{Li}$ & $\mathrm{Au}$ & $\mathrm{Fl}$ & 10 \\
\hline Verbena delticola Small ex L.M. Perry DEB41544 & $\mathrm{Hi}$ & $\mathrm{Au}$ & $\mathrm{Fl}$ & 11 \\
\hline Xolocotzia asperifolia Miranda DN5477, FMG5319 & $\mathrm{Ab}$ & $\mathrm{Au}$ & $\mathrm{Fl}$ & 3,6 \\
\hline \multicolumn{5}{|l|}{ Violaceae } \\
\hline $\begin{array}{l}\text { Hybanthus attenuatus (Humb. \& Bonpl. ex Roem. \& Schult.) Schulze-Menz } \\
\text { DEB10632, JAEJ \& ALP248 }\end{array}$ & $\mathrm{Hi}$ & $\mathrm{Au}$ & $\mathrm{Fl}$ & 7,8 \\
\hline \multicolumn{5}{|l|}{ Viscaceae } \\
\hline $\begin{array}{l}\text { Dendrophthora guatemalensis Standl. DEB30016, DEB, PRH } \\
\qquad \& \text { Ers/n Be, Chis, G }\end{array}$ & $\mathrm{Hi}$ & $\mathrm{Pa}$ & $\mathrm{Fl}$ & 12 \\
\hline Phoradendron nervosum Oliv. JAEJ774 & $\mathrm{Hi}$ & $\mathrm{Pa}$ & $\mathrm{Fr}$ & 6 \\
\hline Phoradendron rondeletiae Trel. DEBs/n Be, Chis, G & $\mathrm{Hi}$ & $\mathrm{Pa}$ & - & 8 \\
\hline Phoradendron treleaseanum Standl. \& Steyerm. DEB27150 & $\mathrm{Hi}$ & $\mathrm{Pa}$ & - & 8 \\
\hline \multicolumn{5}{|l|}{ Vitaceae } \\
\hline Ampelocissus acapulcensis (Kunth) Planch. DEB9050 & $\mathrm{Li}$ & $\mathrm{Au}$ & $\mathrm{Fl}$ & 2 \\
\hline Ampelocissus mesoamericana Lombardi DEB9050 Chis, Hon, S, Ver & $\mathrm{Li}$ & $\mathrm{Au}$ & - & - \\
\hline Cissus biformifolia Standl. JAEJ343 & $\mathrm{Li}$ & $\mathrm{Au}$ & $\mathrm{Fl}$ & 9 \\
\hline Cissus rhombifolia Vahl DEB39924, 52193 & $\mathrm{Li}$ & $\mathrm{Au}$ & $\mathrm{Fl}$ & 8,9 \\
\hline Cissus verticillata (L.) Nicolson \& C.E. Jarvis. DEB, ER \& PH13493, JAEJ81 & $\mathrm{Li}$ & $\mathrm{Au}$ & $\mathrm{Fr}$ & 8,10 \\
\hline Vitis bourgaeana Planch. AM, GUR \& EMS76, DEB \& ARS21539, JAEJ320 & $\mathrm{Li}$ & $\mathrm{Au}$ & $\mathrm{Fr}$ & $2,9,11$ \\
\hline Vitis tiliifolia Humb. \& Bonpl. ex Roem. \& Schult. JAEJ362, MAHM6 & $\mathrm{Li}$ & $\mathrm{Au}$ & $\mathrm{Fr}$ & 4,9 \\
\hline \multicolumn{5}{|l|}{ Zygophyllaceae } \\
\hline Kallstroemia rosei Rydb. HMC9, JAEJ170, JAEJ \& JMM198 & $\mathrm{Hi}$ & $\mathrm{Au}, \mathrm{Ru}$ & $\mathrm{Fl}$ & 7,11 \\
\hline
\end{tabular}


Apéndice 2. Relación de colectores de flora del Parque Nacional Cañón del Sumidero.

\begin{tabular}{|c|c|c|c|}
\hline Colector & Abreviatura & Colector & Abreviatura \\
\hline Alejandra Corzo Domínguez & ACD & Carlos Arturo Toledo Manzur & CATM \\
\hline A. Chamé & $\mathrm{ACH}$ & Carolina A. Zenteno M. & CAZM \\
\hline Alfonso Delgado Salinas & ADS & C.C. Clevinger & CCC \\
\hline A. Espejo & AES & Christian Coello Galindo & CCG \\
\hline Frank Almeda & $\mathrm{AF}$ & C.E. Hughes & $\mathrm{CEH}$ \\
\hline A.F. Moldenke & AFM & Carlos Ernesto Méndez Morales & CEMM \\
\hline Alonso F.S. & AFS & Charles H. Perino & $\mathrm{CHP}$ \\
\hline A. Gómez & AG & Chester Walter Laskowski & ChWL \\
\hline Adrián Gutiérrez González & AGG & Cortes L. & $\mathrm{CL}$ \\
\hline A. Gómez Pompa & AGP & C. Martínez & $\mathrm{CM}$ \\
\hline Anaximandro Gómez Velázquez & AGV & C. M. González Hernández & $\mathrm{CMGH}$ \\
\hline Ana I. Gómez & AIG & Carlos M. Zambrano & CMZ \\
\hline A.I. Gómez B. & AIGB & C. Rojas & CR \\
\hline A. Jack & $\mathrm{AJ}$ & Carmen Soto José & CSJ \\
\hline A.J. Cronquist & $\mathrm{AJC}$ & Carlos Toledo & СТ \\
\hline A. J. Zenón & AJZ & C. Zúñiga & $\mathrm{CZ}$ \\
\hline Ariosto J. Urbina Ballinas & AJUB & C. Zambrano C. & CZC \\
\hline A. Long & $\mathrm{AL}$ & Daniela Ariadna León Gutiérrez & DALG \\
\hline Ana Lucía Anza De La Cruz & ALA & David Dreyer & DDR \\
\hline A.L. Álvarez Chacón & ALAC & D.C. Ledoux & DCLD \\
\hline A.L. Hempel & $\mathrm{ALH}$ & D.C. Saunders & DCS \\
\hline Amaury Márquez & $\mathrm{AM}$ & D.C. Sanders & DCSa \\
\hline A. Montesinos C. & AMC & Deily De León Orozco & DDLO \\
\hline A. Mcdonald & AMD & Dennis E. Breedlove & DEB \\
\hline A. Miller & AMI & Dimitri Flores Girón & DFG \\
\hline A. Maldonado & AML & D.K. Cox & $\mathrm{DKC}$ \\
\hline Ariel Muñoz Núñez & AMN & Daniel León Álvarez & DLA \\
\hline Alonso Méndez Ton & $\mathrm{AMT}$ & David Martin Bates & $\mathrm{DMB}$ \\
\hline A. Pridgeon & $\mathrm{AP}$ & David A. Neill & $\mathrm{DN}$ \\
\hline Angelica Rodríguez & AR & Dunn & DNN \\
\hline Alberto Reyes García & ARG & Dylan P. Hannon & $\mathrm{DPH}$ \\
\hline A.R. López Ferrari & ARLP & D. Rodríguez G. & DRG \\
\hline A.R. Moldenke & ARM & D. Sutton & DS \\
\hline A.Riechers P. & ARP & D.S. Seigler & DSS \\
\hline A. Ramírez R. & ARR & Daniel Torres Reyes & DTR \\
\hline Alan Reed Smith & ARS & E.A. Manrique & EAM \\
\hline A. Salinas T. & AST & E. Cabrera & $\mathrm{EC}$ \\
\hline Acero $\mathrm{T}$. & ATGM & Edgar Cabrera Cano & $\mathrm{EC}$ \\
\hline A.T. Whittemore & ATW & Eugenia Escobar Hernández & $\mathrm{EE}$ \\
\hline A. Vásquez T. & AVT & Eric Hagsater Gartenberg & $\mathrm{EH}$ \\
\hline Bruce Bartholomew & $\mathrm{BB}$ & Emily Jane Lott & EJLTT \\
\hline C. Burns & $\mathrm{BC}$ & E. Lathrop W. & $\mathrm{EL}$ \\
\hline B. Pérez G. & BPG & Ellis & ELL \\
\hline Brown & BRW & Evangelina López Molina & ELM \\
\hline B.T. Keller & BTK & Eizi Matuda & EM \\
\hline César Andrés Pérez Bonifaz & CAPB & Elizabeth Mcclintock & EMC \\
\hline
\end{tabular}


Apéndice 2. Continuación.

\begin{tabular}{|c|c|}
\hline Colector & Abreviatura \\
\hline Esteban Manuel Martínez Salas & EMS \\
\hline Eduardo Palacios Espinosa & EPE \\
\hline E. Raven & ER \\
\hline Emilio Romero Berny & $\mathrm{ERB}$ \\
\hline ND & ERMA \\
\hline Ever Ulises Vazquez Perez & EUVP \\
\hline Fausto Bolom Ton & FBT \\
\hline Fernando Daniel Acosta López & FDAL \\
\hline Francisco Hernández Najarro & $\mathrm{FHN}$ \\
\hline F. Martínez & FM \\
\hline Faustino Miranda González & FMG \\
\hline F. Riveros & $\mathrm{FR}$ \\
\hline F.S. Kawahara & FSK \\
\hline Foster Van Hyning & $\mathrm{FVH}$ \\
\hline Guzmán & G \\
\hline G. Andrade & GA \\
\hline Gerardo A. Salazar & GAS \\
\hline G. Castaño Sub & GC \\
\hline G. Castillo Campos & GCC \\
\hline Gabriela Del Carmen López Hernández & GCLH \\
\hline G. Cortés R. & GCR \\
\hline Gerrit Davidse & GD \\
\hline G. Díaz M. & GDM \\
\hline G. Holstein & $\mathrm{GH}$ \\
\hline Gloria Karina García Ruíz & GKGR \\
\hline G.L. Webster & GLW \\
\hline G. Martínez & GM \\
\hline Gutiérrez M. & GM \\
\hline G. Reyes & GR \\
\hline G. Rodríguez & GRZ \\
\hline Guillermo Urquijo & GUR \\
\hline G.V. Nazart & GVN \\
\hline Helia Bravo H. & $\mathrm{HBH}$ \\
\hline H. De Cabrera & $\mathrm{HC}$ \\
\hline H.E. Trujillo Rios & HETR \\
\hline H. González & HG \\
\hline H. González & $\mathrm{HG}$ \\
\hline Helen Ireland & $\mathrm{HI}$ \\
\hline Humberto Montesinos Castillejos & HMC \\
\hline Héctor Mejia E. & HME \\
\hline H. Pérez U. & $\mathrm{HPU}$ \\
\hline H.S. Gentry & HSG \\
\hline HTR & HTR \\
\hline I. Cacho & IC \\
\hline I. Pérez & IP \\
\hline Iris Velásquez Solís & IVS \\
\hline
\end{tabular}

\begin{tabular}{ll}
\hline Colector & Abreviatura \\
\hline I.V. Solís H. & IVSH \\
Josefa Anahi Espinosa Jiménez & JAEJ \\
J.A. Moguel Acuña & JAMA \\
Jorge A. Vázquez Velázquez & JAVV \\
J. Brunken & JBK \\
Jaime Ballinas Zapata & JBZ \\
Jane Cecil & JC \\
Julio Coutiño Molina & JCM \\
José Carmen Soto Núñez & JCSN \\
Javier Chavelas Pólito & JCP \\
Jeany Davidse & JD \\
J. Flores L. & JFL \\
J. Gómez & JG \\
Jacqueline Guzmán Hernández. & JGH \\
José D. García Pérez & JGP \\
J. Hernández M. & JH \\
Julio Hernández C & JHC \\
J.I. Calzada & JIC \\
J. Jiménez & JJ \\
J.J. Velásquez Jiménez & JJVJ \\
J. La Frankie & JL \\
Jeremias López Chagala & JLCH \\
José L. Linares & JLL \\
J.L. Neff & JLN \\
José Luis Ovilla Hernández & KLA \\
José Luis Panero Estévez & LAH \\
J.L. Pavón T. & LDP \\
John Lance Strother & JEU \\
J.L. Villaseñor R. & JLPE \\
Julia Lazaro Zermeño Hardison & JLPT \\
Jorge Mandujano & JLS \\
J.M. Hill Jr. & JLVR \\
Jorge Martínez Meléndez & JW \\
John Pruski & JLZ \\
José Roque Velásquez & JM \\
J. Rzedowski & JMH \\
J.T. Baldwin Jr. & JMM \\
J.W. Durham & JRV \\
J. Worley & JR \\
\hline
\end{tabular}


Apéndice 2. Continuación.

\begin{tabular}{|c|c|}
\hline Colector & Abreviatura \\
\hline Luis Felipe Martínez Jiménez & LFMJ \\
\hline L. González & LG \\
\hline L. Hernandez S. & $\mathrm{LH}$ \\
\hline L.I. Davis & LID \\
\hline L. Limón & LLI \\
\hline L.M. González V. & LMGV \\
\hline L. Pacheco & LP \\
\hline L. Pérez & LPZ \\
\hline María De Los Angeles Hernández Moreno & MAHM \\
\hline Miguel Alberto Magaña Alejandro \& & MAMA \\
\hline \multicolumn{2}{|l|}{ Grupo Taxonomía } \\
\hline Maguire \& Maguire & MA\&MA \\
\hline M.A. Palacios & MAP \\
\hline Miguel Ángel Pérez Farrera & MAPF \\
\hline Miguel Ángel Soto Arenas & MASA \\
\hline Ma. Ángela Vazquez & MAV \\
\hline Mark Hasslock Mayfield & $\mathrm{MHM}$ \\
\hline Mona Bourell & $\mathrm{MB}$ \\
\hline M. Cházaro B. & $\mathrm{MCHB}$ \\
\hline M.C. López P. & MCLP \\
\hline Maria C. Martínez De López & MCML \\
\hline Marcos David Reynoso Santos & MDRS \\
\hline Marcos E. Becerra & MEB \\
\hline Martinez F & MF \\
\hline M. Flores C. & MFC \\
\hline María G. Díaz & MGD \\
\hline María Guadalupe Rodríguez Guillén & MGRG \\
\hline M.G. Vázquez M. & MGVM \\
\hline M.J. Huft & $\mathrm{MH}$ \\
\hline M. Heath & $\mathrm{MH}$ \\
\hline Maximiano H. P. & MHP \\
\hline María Imelda González López & MIGL \\
\hline M.J. Díaz-Gómez & MJDG \\
\hline Mercedes Jimenez Pavón & MJP \\
\hline M. Kimnach & MK \\
\hline Matt Lavin & ML \\
\hline María Luisa Martínez Jiménez & MLMJ \\
\hline Mahinda Martínez & MM \\
\hline M. Mancilla & MMAN \\
\hline Margarita Mérida & MME \\
\hline M. Martínez Ovando & $\mathrm{MMO}$ \\
\hline M. Negrete A. & MNA \\
\hline Maian N. Farrera Anza & MNFA \\
\hline M. Olson & $\mathrm{MO}$ \\
\hline M. P. Martínez Morales & MPMM \\
\hline M. Palacios Ríos & MPR \\
\hline
\end{tabular}

\begin{tabular}{ll}
\hline Colector & Abreviatura \\
\hline M. Ravelo V. & MRV \\
Martínez S. & MS \\
Mario Sousa Sánchez & MSS \\
N. Bashor & NB \\
Nayely Martínez Meléndez & NMM \\
Neptalí Ramírez Marcial & NRM \\
Oscar F. Clarke & OFC \\
O.G. Enriquez & OGE \\
Oscar Farrera Sarmiento & OFS \\
O. Montesinos & OM \\
Osvaldo Téllez & OT \\
P.A. Fryxell & PAF \\
Pablo Basurto & PB \\
Pilar Carmona L. & PCL \\
P. Cándido Méndez & PCM \\
P.E Glover & PEG \\
P.J. Stafford & PJS \\
P. Magaña & PM \\
Peter Raven Hamilton & PRH \\
P. Robles Z. & PRZ \\
Pedro Tenorio L. & PTL \\
R. Brossard & RB \\
Roberto Canseco Cruz & RCC \\
R. Dorsch & RD \\
Rogelio Ervin Gallegos Ramos & REGR \\
Robert Earle Magill & REM \\
R.E. Weaver & REW \\
Robert Folger Thorne & RTC \\
R. Garcia A. & SAM \\
R. Gutiérrez O. & RFT \\
R. Hampshire & RGA \\
Reyna Karina Rodríguez Martínez & RGO \\
R. Lelua & RGael Torres Colín \\
Robert Louis Dressler & RH \\
R.L. Hartman & RKRM \\
Rubén Martínez Camilo & RL \\
R. Mcvaugh & RLD \\
Robert Merrill King & RLH \\
Robert M. Laughlin & RMC \\
Rosa Ortiz & RMcV \\
Rosa O. Rincón R. & RMK López \\
\hline
\end{tabular}


Apéndice 2. Continuación.

\begin{tabular}{ll}
\hline Colector & Abreviatura \\
\hline S. Sundberg & SBG \\
Scott D. Sundberg & SD \\
Stephen Douglas Koch & SDK \\
Sergio García Sánchez & SGS \\
Sergio Zamudio Ruíz & SZR \\
Silvia Guadalupe Trujillo & SGT \\
Santiago Rosales & SR \\
Trinidad Alemán Santillán & TAS \\
Thomas B. Croat & TBC \\
Thomas Baillie Macdougall & TBMc \\
Thomas Franklin Daniel & TFD \\
Thomas Leighton Wendt & TLW \\
Thennilapuram Parasuraman Ramammoorthy & TPR \\
Thomas R. Soderstrom & TRS \\
\hline
\end{tabular}

\begin{tabular}{ll}
\hline Colector & Abreviatura \\
\hline V.A. Funk & VAF \\
Victor Alonso Gomez Lopez & VAGL \\
V. Mendez & VM \\
V.M. Merida & VMM \\
Viridiana Martínez Martínez & VMM \\
V.M. Sánchez León & VMSL \\
Víctor W. Steinmann & VWS \\
Walter Acuña Castro & WAC \\
Walter C. Holmes & WCH \\
Anderson William (Bill) Russell & WRA \\
C. Wells & WWS \\
Y. Kim & YKM \\
Y Nazarat & YN \\
Yadira Nazart G. & YNG \\
\hline
\end{tabular}

